\title{
Hysteropexy in the treatment of uterine prolapse
}

Citation for published version (APA):

van IJsselmuiden, M. (2020). Hysteropexy in the treatment of uterine prolapse. [Doctoral Thesis, Maastricht University]. Gildeprint Drukkerijen. https://doi.org/10.26481/dis.20200909mi

Document status and date:

Published: 01/01/2020

DOI:

10.26481/dis.20200909mi

Document Version:

Publisher's PDF, also known as Version of record

\section{Please check the document version of this publication:}

- A submitted manuscript is the version of the article upon submission and before peer-review. There can be important differences between the submitted version and the official published version of record.

People interested in the research are advised to contact the author for the final version of the publication, or visit the DOI to the publisher's website.

- The final author version and the galley proof are versions of the publication after peer review.

- The final published version features the final layout of the paper including the volume, issue and page numbers.

Link to publication

\footnotetext{
General rights rights.

- You may freely distribute the URL identifying the publication in the public portal. please follow below link for the End User Agreement:

www.umlib.nl/taverne-license

Take down policy

If you believe that this document breaches copyright please contact us at:

repository@maastrichtuniversity.nl

providing details and we will investigate your claim.
}

Copyright and moral rights for the publications made accessible in the public portal are retained by the authors and/or other copyright owners and it is a condition of accessing publications that users recognise and abide by the legal requirements associated with these

- Users may download and print one copy of any publication from the public portal for the purpose of private study or research.

- You may not further distribute the material or use it for any profit-making activity or commercial gain

If the publication is distributed under the terms of Article $25 \mathrm{fa}$ of the Dutch Copyright Act, indicated by the "Taverne" license above, 


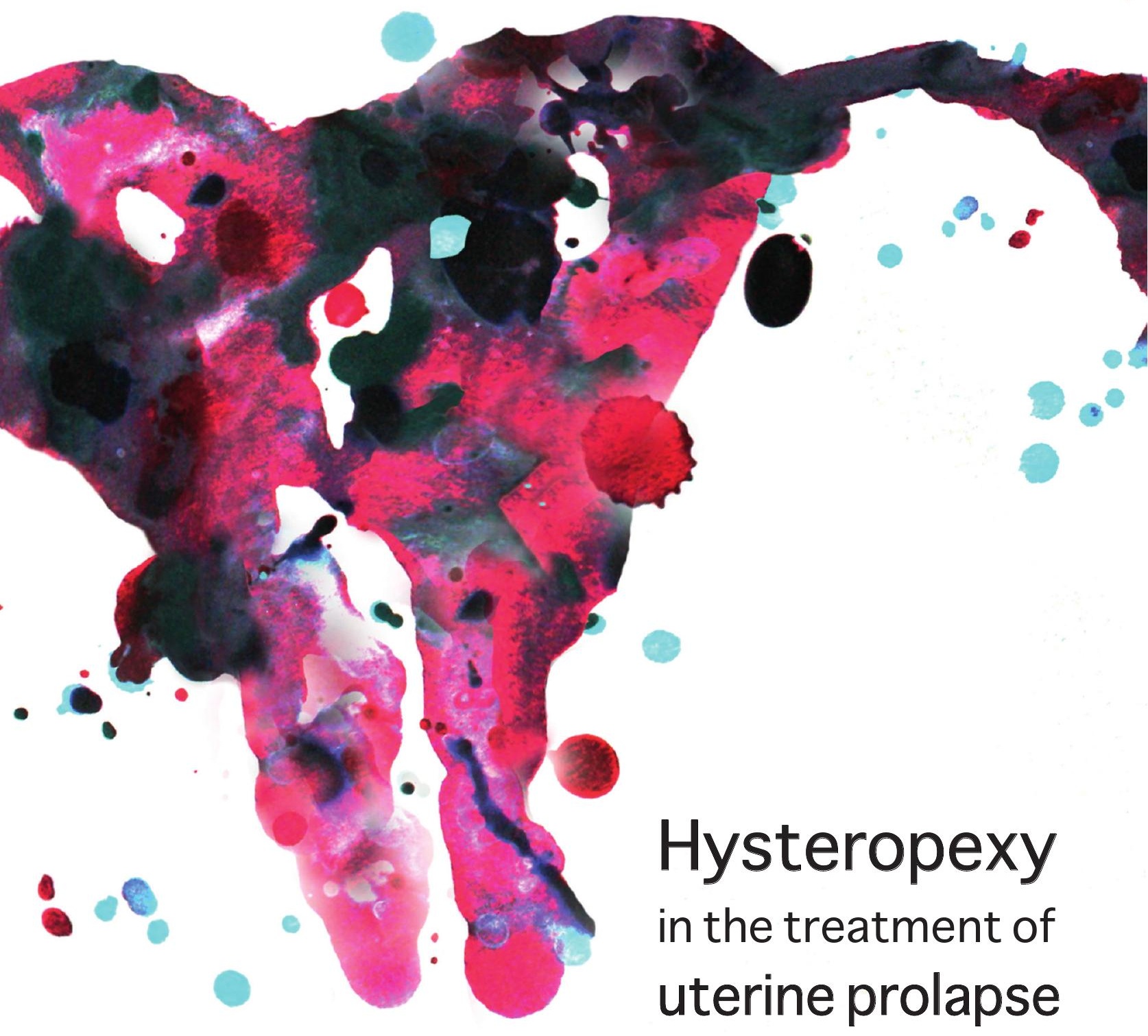

Mèlanie N. van IJsselmuiden 
HYSTEROPEXY IN THE TREATMENT OF UTERINE PROLAPSE 


\section{HYSTEROPEXY IN THE TREATMENT OF UTERINE PROLAPSE}

Hysteropexy in the treatment of uterine prolapse

Thesis, Maastricht University, The Netherlands

ISBN: 978-94-6402-193-6

Cover design: Sanne Bisschop-Hein

Lay-out: Ilse Modder | www.ilsemodder.nl

Printed by: Gildeprint, Enschede | www.gildeprint.nl

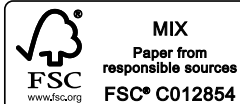

All rights reserved. No part of this thesis may be reproduced, stored in a retrieval system, or transmitted in any form by any means without permission from the holder of the copyright.

The research in this thesis was supported by a grant from the Isala Innovation and Research fund, which is gratefully acknowledged.

Financial support for printing of this thesis was kindly provided by:

Wetenschapsfonds Medisch Specialisten Isala

Applied Medical Europe, Bridea Medical, Chipsoft BV, Coloplast BV, Erbe Nederland BV,

Goodlife Pharma, Karl Storz Endoscopie Nederland BV, Medical Dynamics BV, Medsim

BV, Memidis Pharma BV, Pelvitec BV.

(C) Copyright 2020 MN van IJsselmuiden
PROEFSCHRIFT

ter verkrijging van de graad van doctor aan de Universiteit Maastricht op gezag van de Rector Magnificus, Prof. dr. R.M. Letschert

volgens het besluit van het College van Decanen in het openbaar te verdedigen

op woensdag 9 september 2020 om 14:00 uur

door

Mèlanie Nathalie van IJsselmuiden 


\section{PROMOTOR:}

Prof. dr. M.Y. Bongers

Prof. dr. J. Deprest (Universiteit Leuven, België)

\section{CO-PROMOTOR:}

Dr. H.W.F. van Eijndhoven (Isala Zwolle)

\section{BEOORDELINGSCOMMISSIE:}

Prof. dr. R.F.P.M. Kruitwagen (voorzitter)

Dr. M.H. Blanker (Universitair Medisch Centrum Groningen, Groningen)

Prof. dr. N.D. Bouvy

Dr. R.A. Hakvoort (Martini Ziekenhuis, Groningen)

Voor mijn vader

"Je kunt altijd meer dan je zelf denkt" 


\section{TABLE OF CONTENTS}

CHAPTER 2 Practice pattern variation in surgical management of

pelvic organ prolapse: a Dutch survey.

Int Urogynecol J. 2015 May;26(5):757-64

Euro J Obstet Gynaecol Reprod Biol. 2017 Nov 21;220:79-83

CHAPTER 5 Hysteropexy in treatment of uterine prolapse stage 2 or higher: a multicenter randomized controlled noninferiority trial comparing laparoscopic sacrohysteropexy with vaginal sacrospinous hysteropexy (LAVA-trial, study protocol).

BMC Women's Health. 2014 Sep 17:14:112

CHAPTER 6 Hysteropexy in the treatment of uterine prolapse stage 2 or higher: laparoscopic sacrohysteropexy versus sacrospinous hysteropexy - a multicentre randomized controlled trial (LAVA trial)

BJOG. 2020 doi: 10.1111/1471-0528.16242.

CHAPTER 7 Dynamic magnetic resonance imaging to quantify pelvic organ mobility after treatment for uterine descent: differences between surgical procedures Int Urogynecol J. 2020 doi: 10.1007/s00192-020-04278-5. 


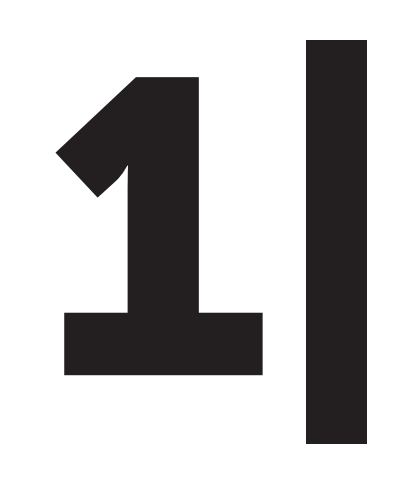

GENERAL INTRODUCTION AND OUTLINE OF THIS THESIS 


\section{PELVIC ORGAN PROLAPSE}

Pelvic organ prolapse (POP) is defined as the downward displacement of one of the pelvic organs including the uterus and/or different vaginal compartments and their neighbouring organs such as bladder, rectum or bowel [1]. POP is part of the group of pelvic floor disorders: clinical conditions caused by dysfunction of the pelvic floor, which comprises urinary incontinence, lower urinary tract symptoms, faecal incontinence, defecatory dysfunction, chronic pain syndromes and sexual dysfunction.

Pelvic organ support depends on the combination of function of connective tissue attachments (endopelvic fascia), tensile strength of the tissues and muscular support (levator ani muscle). This support is empirically divided by three levels (figure 1) [2]:

- Level I: support of the upper third of the vagina/uterus by the uterosacral and cardinal ligaments to the sacrum and the lateral pelvic side wall

- Level II: paravaginal attachments of the middle half of the vagina to the arcus tendineus fascia pelvis

- Level III: the fusion of the lower third of the vagina to the perineal membrane and perineal body

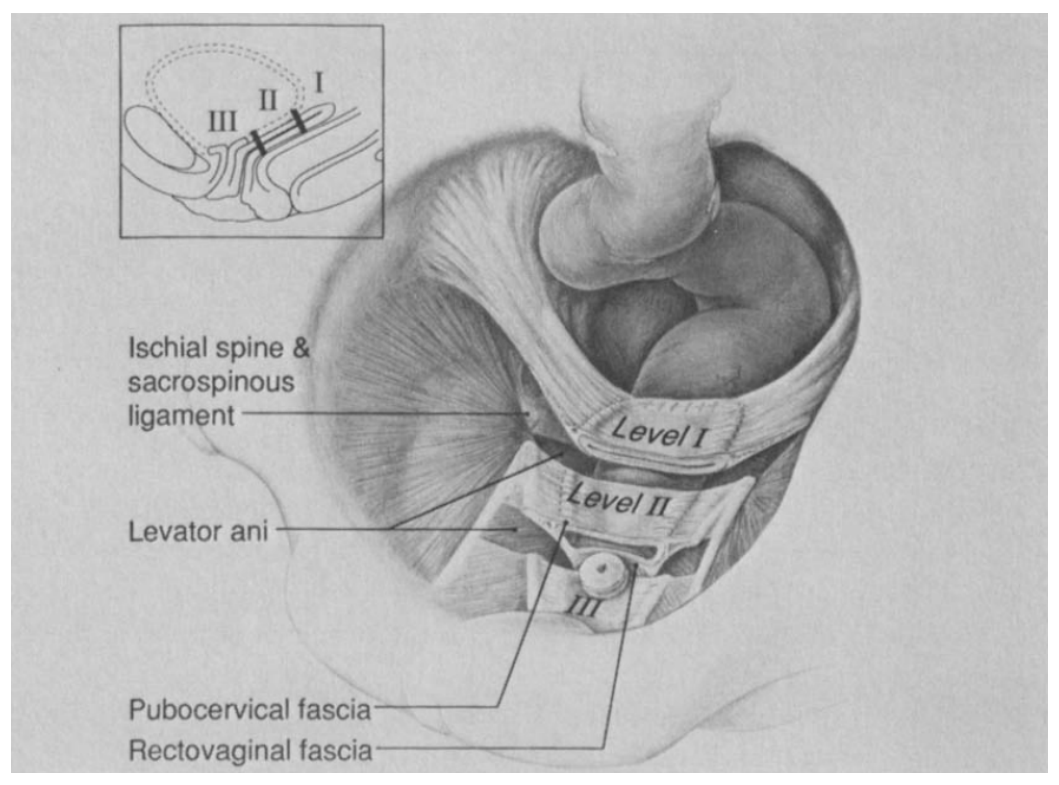

FIGURE 1. Biomechanical models by DeLancey. Level I: proximal suspension, level II: lateral attachment, level III: distal fusion. (From DeLancey JO. Anatomic aspects of vaginal eversion after hysterectomy. Am J Obstet Gynecol 1992;166:1717).
Disruption of one of these structures can lead to loss of support and POP. Depending on the location and function of the defect and the organs involved, several types of prolapse can occur. The vagina can be separated into three compartments: the anterior, apical and posterior compartment. Prolapse of the anterior vaginal wall leads to prolapse of the bladder and/or urethra, and is called a (urethro)cystocele. Prolapse of the apical compartment is referred to as uterine or cervical descent (if the uterus or cervix is still in place) or, after vaginal hysterectomy, vaginal vault prolapse. Prolapse of the posterior vaginal wall leads to anterior prolapse of rectum and or small bowel referred as a rectocele or enterocele respectively. POP can be present in one compartment, but usually more compartments are affected simultaneously.

$\mathrm{POP}$ is one of the most common gynaecological conditions. In a Dutch cross-sectional study, $40 \%$ of the women over 45 years of age had an anatomical prolapse stage two or higher on clinical examination [3]. However, in the vast majority the prolapse was asymptomatic, only $12.1 \%$ reported a seeing and/or feeling of vaginal bulging. The lifetime risk of undergoing surgery for POP or stress urinary incontinence is estimated to be $20 \%$ by the age of 80 years [4]. As result of the aging of the population, a significant increase in both the number of women with POP and those seeking care for POP is expected to occur over the next 20-40 years [5]. Consequently, the surgical rates for POP and stress urinary incontinence in the United States are estimated to increase with $42.7 \%$ by 2050 [5].

The aetiology of POP is multifactorial. Several risk factors are established, including higher parity, vaginal childbirth, advancing age, obesity and previous hysterectomy. Potential risk factors include forceps delivery, high infant birth weight (> $4500 \mathrm{~g}$ ), prolonged second stage of labor, age $<25$ years at first delivery, pregnancy itself, family history of POP, occupations entailing heavy lifting, constipation and connective tissue disorders [6].

Although POP is not a life-threatening condition, it significantly affects a woman's quality of life and may influence urinary, gastrointestinal, sexual and psychological functioning. In a Dutch cross-sectional study, symptomatic prolapse was found in $11.4 \%$ of the women aged over 45 years [7]. The correlation between prolapse symptoms and the stage of prolapse is weak [8]. However, the one symptom that is consistently mentioned by patients with advanced POP is seeing or feeling a vaginal bulge [9]. In addition, the hymen seems to be an important threshold for symptoms as women with prolapse beyond the hymen have more POP symptoms and are more likely to report a vaginal bulge as compared to women with prolapse at or above the hymen [10] 


\section{EXAMINATION AND CLINICAL QUANTIFICATION}

In order to standardize and quantify the degree of POP, the Pelvic Organ Prolapse Quantification system (POP-Q) has been developed [11]. During vaginal examination, maximum prolapse is demonstrated and identified by asking the patient to cough or to perform a Valsalva manoeuvre while each vaginal wall is individually exposed. The degree of prolapse of the anterior vaginal wall (point $\mathrm{Aa}$ and $\mathrm{Ba}$ ), the posterior vaginal wall (point $A p$ and $B p$ ) and the cervix (point $C$ and $D$ ) are measured in centimetres either above/proximal to the hymen (negative number) or beyond/distal to the hymen (positive number). The plane of the hymen is defined as zero. Furthermore, the perineal body (Pb), the genital hiatus ( $\mathrm{gh}$ ) and the total vaginal length (tvl) are measured. Based on these measurements, a stage (0-4) is determined for each compartment whereas stage 4 is classified as complete eversion.

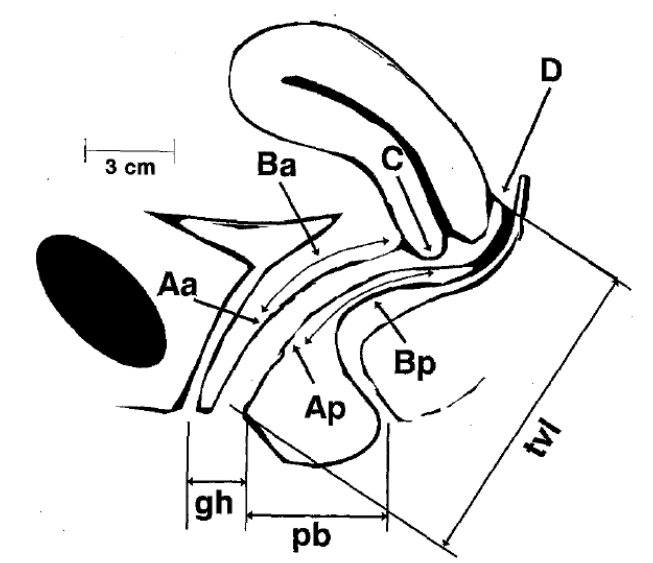

FIGURE 2. POP-Q examination (From Bump RC et al. The standardization of terminology of female pelvic organ prolapse and pelvic floor dysfunction. Am J Obstet Gynecol 1996;175:10)

\section{CONSERVATIVE TREATMENT}

Treatment of POP is only indicated if a patient has complaints for which she seeks therapy. There are rare cases of advanced prolapse that compromise renal function by ureteral obstruction, mandating intervention to maintain renal function, or vaginal bleeding due to erosive lesions, but outside of these no absolute indication for intervention exists [12]. It is not known if surgery performed at an early or even asymptomatic stage is more effective than an intervention after the onset of symptoms or worsening of prolapse stage. Therefore, watchful waiting could be a reasonable option in women with asymptomatic prolapse or women with mild POP symptoms.
In studies on natural history of POP, only a minimal change over time has been documented [12-14]. In a retrospective study with a median follow-up of 16 months, $63 \%$ of the women preferring watchful waiting continued to do so. The main reason to ask for an intervention was the occurrence or worsening of prolapse symptoms. Over time, $78 \%$ of the patients showed no anatomical change in POP-Q examination [12].

POP can be treated conservatively or surgically. The choice of treatment depends on the preference of the woman and the gynaecologist. Clearly defined guidelines regarding indications for conservative or surgical treatment of POP are lacking. In general, according to the Dutch guideline, it is advised to start with conservative interventions; surgical therapy is indicated after failure of conservative treatments. However, preference of the woman is leading [15].

Conservative treatment is defined as any treatment that does not involve medication or surgical treatment, such as life style interventions, physical therapies and/or pessaries [16] . Life style interventions include weight loss, reducing activities that strain the pelvic floor and treating constipation to avoid further exacerbation of the prolapse by decreasing intra-abdominal pressure. There are no studies evaluating the long-term effectiveness of life style interventions in the treatment of POP [16]

Several studies have demonstrated efficacy of pelvic floor muscle training (PFMT) in the treatment of POP, both as a treatment in itself and as an adjunct to surgical therapy. It is believed that the effect of PFMT is due to learning women 1) to consciously contract their pelvic floor muscles before and during increase in abdominal pressure and 2) to improve structural functional support by the performance of pelvic floor exercises [17]. PFMT may reduce the symptoms and severity of prolapse and as a result improve quality of life [18-20]. The efficacy of PFMT is mainly observed in low stage prolapse (stage I or II), studies assessing PFMT in advanced stage prolapse are lacking.

Pessaries are another non-surgical option for the treatment of POP. Pessaries can be categorized in two types: supporting type (e.g. ring pessaries) and space-occupying type (e.g. gellhorn pessaries). In general, supporting pessaries are used in patients with mild prolapse (stage I or II), whereas space-occupying pessaries are typically used in advanced prolapse (stage III or IV) if resistant to the supporting type. There is no difference in efficacy between pessary treatment and PFMT in reducing pelvic floor symptoms, but specific prolapse-related symptoms seem to improve more with pessary treatment. In addition, pessary treatment is more cost-effective [21]. However, pessaries fit less good in $43 \%$ of women. Predictors of unsuccessful pessary 
fitting are higher $\mathrm{BMl}$, older patients, shorter transvaginal length $(<7.3 \mathrm{~cm})$, previous hysterectomy and underactive pelvic floor muscles [22-24]. Furthermore, pessary use is associated with more side effects as compared to PFMT (e.g. increased vaginal discharge, increase in urinary incontinence and irritation or erosions of the vaginal walls on physical examination) [21]. The pessary discontinuation rate varies from $17 \%$ after 1 year to $24-86 \%$ after 5 or more years [25-28]. Side effects occur in more than half of women and are the main reason for discontinuation [28,29].

\section{SURGICAL TREATMENT}

The main goals of POP surgery are to restore normal pelvic anatomy, eliminate POP symptoms and normalize bowel, bladder and sexual function [6]. Surgery for prolapse can be performed by either the vaginal or abdominal route. The latter can be subdivided into open abdominal, laparoscopic or robotically assisted surgery. The vaginal route is carried out in $80-90 \%$ of all procedures $[30,31]$. Clearly defined guidelines on surgical POP repair are lacking. The choice of surgery depends on several factors including site and severity of the prolapse, general health of the woman, expertise of the surgeon and the preference of both patient and surgeon.

Unfortunately, surgical prolapse procedures are associated with a high reoperation rate. A Danish population-based registry study demonstrated a cumulated incidence rate of reoperation for POP in the anterior, apical and posterior compartment of $12.4 \%, 7.9 \%$ and $12.1 \%$ respectively [32]. Overall the reoperation rate was $11.5 \%$. A peak was demonstrated in the group of women who had their primary surgery before the menopause (18-49 years, 26.9\%) as compared to the women between 50-90 years (10.1\%).

\section{Surgical treatment of uterine descent}

Traditionally, uterine prolapse is treated by vaginal hysterectomy and suspension of the vaginal vault, despite the fact that the uterus is not the cause but only a passive structure in the development of prolapse $[2,33]$. While anterior vaginal wall prolapse is the most common site of prolapse, loss of apical support is usually present in case the prolapse extends beyond the hymen $[34,35]$. Therefore, adequate support of vaginal apex (level I support) is an essential component of a durable surgical repair of advanced prolapse. Vaginal hysterectomy alone gives no adequate apical support and additional suspension of the vaginal apex after hysterectomy is recommended [36]. Uterus preserving techniques are becoming more popular [37-40]. In general, uterinepreserving surgeries improve operating time and blood loss compared to similar procedures with concomitant hysterectomy without significant change in short-term prolapse recurrence [41]. The sacrospinous hysteropexy and the Manchester-Fothergill (both vaginal) and abdominal sacrohysteropexy (open abdominal/laparoscopic/ robotic assisted) are the most used uterus preserving techniques.

Several randomized controlled trials compared anatomical and functional outcome after vaginal sacrospinous hysteropexy and vaginal hysterectomy with suspension of the uterosacral ligaments in women with uterine descent [42-44]. In general, the outcome of both procedures was comparable. However, at five year follow-up, significantly less anatomical recurrences of the apical compartment with bothersome bulge symptoms or repeat surgery were found after vaginal sacrospinous hysteropexy compared to vaginal hysterectomy with uterosacral ligament suspension [44].

Another procedure for uterine descent with uterine preservation is laparoscopic sacrohysteropexy. The effectiveness of laparoscopic sacrohysteropexy in treatment of uterine prolapse was demonstrated in several studies. At short-term follow-up, the procedure was successful in 50 out of 51 women, with no objective evidence of uterine prolapse on examination [45]. High patient satisfaction with low rates of apical prolapse recurrence was found at mean follow-up of 2 years [46]. Furthermore, the risk of repeat apical surgery at one year after laparoscopic sacrohysteropexy was similar as compared to vaginal hysterectomy, suggesting this procedure is a safe surgical alternative to vaginal hysterectomy [47].

Vaginal sacrospinous hysteropexy is the most studied surgical technique for uterine descent in the Netherlands and the procedure is very popular among Dutch gynaecologists. However, there are some comments on this technique. After vaginal sacrospinous hysteropexy, the vaginal axis is changed to a more horizontal and posterior position $[48,49]$. Furthermore, the high risk of anterior vaginal wall prolapse after this technique is often discussed [50,51]. As for anatomic recurrence after vaginal sacrospinous hysteropexy, the cervix generally remains well fixed to the sacrospinous ligament, but the weak point is supposed to be the anterior compartment [52]. It is hypothesized that the high rates of recurrence in the anterior vaginal wall after vaginal sacrospinous hysteropexy may be related to the previously incurred damage of muscular supports (e.g. levator ani muscle injury, reduced muscle strength), the change in vaginal axis or a combination of these two $[50,53]$. Abdominal or laparoscopic uterine suspension might not have these disadvantages.

In case of vaginal vault prolapse, abdominal sacrocolpopexy has been shown to have a lower rate of recurrent apical prolapse and a lower reoperation rate as compared 
to vaginal sacrospinous fixation of the vaginal vault [35]. When compared to vaginal POP surgery of the vaginal vault in general (vaginal sacrospinous fixation of the vault, uterosacral suspension of the vault and transvaginal mesh), sacrocolpopexy is associated with lower risk of any recurrent prolapse on examination, repeat surgery for prolapse, postoperative stress urinary incontinence and dyspareunia [35]. With the advantages of sacrocolpopexy of the vaginal vault, laparoscopic hysteropexy is a promising surgical technique. Consequently, this procedure seems to become increasingly popular [38,54]. However, laparoscopic sacrohysteropexy has not been compared directly in a randomised trial against vaginal sacrospinous hysteropexy. As a result, it is unclear whether or not these uterine suspension procedures have comparable anatomical and functional outcomes.

\section{OUTLINE OF THIS THESIS}

This thesis studies different treatment methods for uterine prolapse. Emphasis is placed on the comparison of vaginal and laparoscopic hysteropexy. The following objectives are addressed:

- How do doctors in the Netherlands treat uterine prolapse? And more specific, how do Dutch gynaecologists perform laparoscopic sacrohysteropexy and sacrocolpopexy?

- What treatment do women in the Netherlands prefer (uterus preservation or removal) when surgical management for uterine descent is indicated?

- How should women with uterine prolapse be treated and is there a difference in outcomes between laparoscopic and vaginal hysteropexy?

- Is there a difference in pelvic mobility after sacrospinous hysteropexy as compared to other treatments for uterine prolapse?

The availability of many treatment modalities for POP can lead to variation in care between physicians and hospitals, referred to as practice pattern variation. In CHAPTER 2 we describe the practice pattern variation for treating POP patients in Dutch hospitals.

A surgical technique of a specific procedure might vary between gynaecologists as well. CHAPTER 3 describes variation between Dutch gynaecologists in surgical technique of the laparoscopic sacrohysteropexy and laparoscopic sacrocolpopexy.

It is believed that uterine preservation is becoming more popular because women want to retain their uterus more often [37,38]. However, evidence to support this statement is lacking. In CHAPTER 4 women's attitude towards hysterectomy and uterus preservation in surgical management of uterine prolapse is described.

CHAPTER 5 provides a study protocol of a randomized controlled clinical trial to investigate outcomes after laparoscopic sacrohysteropexy and vaginal sacrospinous hysteropexy.

CHAPTER 6 presents the results of that randomized study comparing laparoscopic sacrohysteropexy and vaginal sacrospinous hysteropexy in the treatment of uterine prolapse (LAVA trial). Surgical failure, functional outcome and quality of life were evaluated after 12 months follow-up.

.


In the literature, the high risk for recurrent prolapse of the anterior vaginal wall after vaginal sacrospinous hysteropexy is often discussed. In order to find an explanation, in CHAPTER 7 vaginal axes and pelvic mobility after vaginal sacrospinous hysteropexy were assessed by dynamic magnetic resonance imaging and compared to other surgical techniques for uterine descent (laparoscopic sacrohysteropexy and vaginal hysterectomy).

CHAPTER 8 contains the general discussion, clinical implications and future perspectives.

In CHAPTER 9 AND 10 a summary in English and Dutch is given.

\section{REFERENCES}

1. Haylen BT, Maher CF, Barber MD, Camergo S, Dandolu V, Digesu A, Goldman HB, Huser M, Milani AL, Moran PA, Schaer GN, Withagen MI. An international Urogynecological Association (IUGA)/International Continence Society (ICS) joint report on the terminology for female pelvic organ prolapse (POP). Int Urogynecol J 2016;27(4):655-84

2. DeLancey JO. Anatomic aspects of vaginal eversion after hysterectomy. Am J Obstet Gynecol 1992;166:1717

3. Slieker-ten Hove MC, Pool-Goudzwaard AL, Eijkemans MJ, Steegers-Theunissen RP, Burger CW, Vierhout ME. The prevalence of pelvic organ prolapse symptoms and signs and their relation with bladder and bowel disorders in a general female population. Int Urogynecol J Pelvic Floor Dysfunct 2009;20(9):1037-45

4. Wu JM, Matthews CA, Conover MM, Pate V, Jonsson Funk M. Lifetime risk of stress urinary incontinence or pelvic organ prolapse surgery. Obstet Gynecol 2014;123(6):1201-6

5. Dieter AA, Wilkins MF, Wu JM. Epidemiological trends and future care needs for pelvic floor disorders. Curr Opin Obstet Gynecol 2015;27(5):380-4

6. Barber MD. Pelvic Organ Prolapse. BMJ 2016;354:13853

7. Slieker-ten Hove MC, Pool-Goudzwaard AL, Eijkemans MJ, Steegers-Theunissen RP, Burger CW, Vierhout ME. Symptomatic pelvic organ prolapse and possible risk factors in a general population. Am J Obstet Gynecol 2009;200(2):184

8. Ellerkmann RM, Cundiff GW, Melick CF, Nihira MA, Leffler K, Bent AE. Correlation of symptoms with location and severity of pelvic organ prolapse. Am J Obstet Gynecol 2001:185(6):1332-7

9. Barber MD, Brubaker L, Nygaard I, Wheeler TL 2nd, Schaffer J, Chen Z, Spino C. Defining success after surgery for pelvic organ prolapse. Obstet Gynecol 2009;114(3):600-9

10. Barber MD, Maher C. Epidemiology and outcome assessment of pelvic organ prolapse. Int Urogynecol J 2013;24(11):1783-90

11. Bump RC, Mattiasson A, Bø K, Brubaker LP, DeLancey JO, Klarskov P, Shull BL, Smith AR. The standardization of terminology of female pelvic organ prolapse and pelvic floor dysfunction. Am J Obstet Gynecol 1996:175:10

12. Gilchrist AS, Campbell W, Steele H, Brazell H, Foote J, Swift S. Outcomes of observation as therapy for pelvic organ prolapse: a study in the natural history of pelvic organ prolapse. Neurourol Urodyn 2013;32(4):383-6

13. Miedel A, EK M,Tegerstedt $G$, Maehle-Schmidt $M$, Nyren $\mathrm{O}$, Hammarstrom M. Short-term natural history in women with symptoms indicative of pelvic organ prolapse. Int Urogynecol J 2011;22(4):461-8

14. Handa VL, Garrett E, Hendrix S, Gold E, Robbins J. Progression and remission of pelvic organ prolapse: a longitudinal study of menopausal women. Am J Obstet Gynecol 2004;190(1):27-32

15. Roovers JP, Everhardt E, Dietz V, Milani AL, Meier AHP, Consten ECJ. Richtlijn prolaps. Dutch Society of Obstetry and Gynecology, www.nvog. $\mathrm{nl}, 2014$

16. Dumoulin C, Hunter KF, Moore K, Bradley CS, Burgio KL, Hagen S, Imamura M, Thakar R, Wiliams K, Chambers T. Conservative management for female urinary incontinence and pelvic 
organ prolapse review 2013: summary of the $5^{\text {th }}$ international consultation on incontinence. Neurourol Urodyn 2016;35(1):15-20

17. Bø K. Can pelvic floor muscle training prevent and treat pelvic organ prolapse? Acta Obstet Gynecol Scand 2006:85:263-8

18. Li C, Gong Y, Wang B. The efficacy of pelvic floor muscle training for pelvic organ prolapse: a systematic review and meta-analysis. Int Urogynecol J 2016;27(7):981-92

19. Hagen S, Stark D. Conservative prevention and management of pelvic organ prolapse in women. Cochrane Database Syst Rev 2011; 7(12):CD003882

20. Wiegersma M, Panman CM, Kollen BJ, Berger MY, Lisman-van Leeuwen Y, Dekker JH. Effect of pelvic floor muscle training compared with watchful waiting in older women with symptomatic mild pelvic organ prolapse: a randomized controlled trial in primary care. BMJ 2014;349:97378

21. Panman $C M$, Wiegersma $M$, Kollen $B J$, Berger MY, Lisman-van Leeuwen $Y$, Vermeulen KM, Dekker JH. Effectiveness and cost-effectiveness of pessary treatment compared with pelvic floor muscle training in older women with pelvic organ prolapse: 2-year follow-up of a randomized controlled trial in primary care. Menopause 2016;23(12):1307-1318

22. Markle D, Skoczylas L, Goldsmith C, Noblett K. Patient characteristics associated with a successful pessary fitting. Female Pelvic Med Reconstr Surg 2011;17(5):249-52

23. Panman CM, Wiegersma M, Kollen BJ, Burger $H$, Berger MY, Dekker JH. Predictors of unsuccessful pessary fitting in women with prolapse: a crosssectional study in general practice. Int Urogynecol J 2017; 28(2):307-13.

24. Mao M, Ai F, Zhang Y, Kang J, Liang S, Xu T, Zhu
L. Predictors for unsuccessful pessary fitting in women with symptomatic pelvic organ prolapse a prospective study. BJOG 2018:125(11):1434-40

25. Manonai J, Sarit-Apirak S, Udomsubpayakul U. Vaginal ring pessary use for pelvic organ prolapse: continuation rates and predictors of continued use. Menopause 2018;26(6):665-9

26. Yimphong T, Temtanakitpaisan T, Buppasir P, Chongsomchai C, Kanchaiyaphum S. Discontinuation rate and adverse events after 1 year of vaginal pessary use in women with pelvic organ prolapse. Int Urogynecol J 2018;29(8):1123-

27. Wolff $B$, Williams K, Winkler A, Lind L, Shalom D. Pessary types and discontinuation rates in patients with advanced pelvic organ prolapse. Int Urogynecol J 2017;28(7):993-7

28. Sarma S, Yiing T, Moore KH. Long-term vaginal ring pessary use: discontinuation rates and adverse events. BJOG 2009;116(13)1715-21

29. Broens-Oostveen MC, Mom RM, Lagro-Janssen AL. De genitale prolaps: behandeling en beloop in vier huisartspraktijken. Ned Tijdschr Geneesk 2004;148(29):1444-8

30. Olsen AL, Smith VJ, Bergstrom JO, Colling JC, Clark AL. Epidemiology of surgically managed pelvic organ prolapse and urinary incontinence. Obstet Gynecol 1997;89:501-6

31. Brown JS, Waetjen LE, Subak LL, Thom DH, van den Eeden S, Vittinghoff E. Pelvic organ prolapse surgery in the United States. Am J Obstet Gynecol 2002;186:712-16

32. Lowenstein E, Moller LA, Laigaard J, Gimbel H. Reoperation for pelvic organ prolapse: A Danish cohort study with 15-20 years' follow-up. Int Urogynecol J 2018;29(1):119-24

33. Gutman R, Maher C. Uterine-preserving POP surgery. Int Urogynecol J 2013;24(11):1803-13
34. Brubaker L, Glazener C, Jacquetin B, Maher C, Melgrem A, Norton P, Rajamaheshwari N Theobald P. Surgery for Pelvic Organ Prolapse. 4th International Consultation on Incontinence. Edited by P. Abrams, L. Cordozo, S. Koury and A. Wein Paris 2009; Chapter 15:1278

35. Maher C, Feiner B, Baessler K, ChristmannSchmid C, Haya N, Brown J. Surgery for women with apical prolapse. Cochrane Database Syst Rev 2018:10:CD012376

36. Kantartzis KL, Turner LC, Shepherd JP, Wang L, Winger DG, Lowder JL. Apical support at the time of hysterectomy for uterovaginal prolapse. Int Urogynecol J 2015:26(2):207-12

37. Madsen AM, Raker C, Sung VW. Trends in hysteropexy and apical support for uterovaginal prolapse in the united states from 2002 to 2012. Female Pelvic Med Reconstr Surg 2017:23(6):365-371

38. Jha S, Cutner A, Moran P. The UK national prolapse survey: 10 years on. Int Urogynecol J 2018;29(6):795-801

39. Wu MP, Long CY, Huang KH, Chu CC, Liang CC, Tang $\mathrm{CH}$. Changing trends of surgical approaches for uterine prolapse: an 11-year population based nationwide descriptive study. Int Urogynecol J 2012;23(7):865-72.

40. Detollenaere RJ, den Boon J, Kluivers KB, Vierhout $M E$, van Eijndhoven HW. Surgical management of pelvic organ prolapse and uterine descent in the Netherlands. Int Urogynecol J 2013;24(5):781-8

41. Meriwether KV, Antosh DD, Olivera CK, KimFine S, Balk EM, Murphy M, Grimes CL, Sleemi A. Singh R, Dieter AA, Crisp CC, Rahn DD. Uterine preservation vs hysterectomy in pelvic organ prolapse surgery: a systematic review with metaanalysis and clincal practice guidelines. Am J Obstet Gynecol 2018;219(2):129-46
42. Dietz $\mathrm{V}$, van der Vaart $\mathrm{CH}$, van der Graaf $\mathrm{Y}$, Heintz P. Schraffordt Koops SE. One-year follow-up after sacrospinous hysteropexy and vaginal hysterectomy for uterine descent: a randomized study. Int Urogynecol J 2010;21(2):209-16

43. Detollenaere RJ, den Boon J, Stekelenburg J, IntHout J, Vierhout ME, Kluivers KB et al. Sacrospinous hysteropexy versus vaginal hysterectomy with suspension of the uterosacral ligaments in women with uterine prolapse stage 2 or higher: multicentre randomised non-inferiority trial. BMJ 2015;351:h3717

44. Schulten SFM, Detollenaere RJ, Stekelenburg J, IntHout J, Kluivers KB, van Eijndhoven HWF. Sacrospinous hysteropexy versus vaginal hysterectomy with uterosacral ligament suspension in women with uterine prolapse stage 2 or higher: observational follow-up of a multicentre randomized trial. BMJ 2019;366:15149

45. Price N, Slack A, Jackson SR. Laparoscopic hysteropexy: the initial results of a uterine suspension procedure for uterovaginal prolapse. BJOG 2010;117(1):62-8

46. Rahmanou P, White B, Price N, Jackson S. Laparoscopic hysteropexy: 1- to 4-year followup of women postoperatively. Int Urogynecol J 2014;25(1):131-8

47. Rahmanou P, Price N, Jackson SR. Laparoscopic hysteropexy versus vaginal hysterectomy for the treatment of uterovaginal prolapse: a prospective randomized pilot study. Int Urogynecol J 2015;26(11):1687-94

48. Sze EH, Meranus J, Kohli N, Miklos JR, Karram MM. Vaginal configuration on MRI after abdominal sacrocolpopexy and sacrospinous ligament suspension. Int Urogynecol J Pelvic Floor Dysfunct 2001:12:375-9

49. Rane A, Lim YN, Withey G, Muller R. Magnetic 
resonance imaging findings following three different vaginal vault prolapse repair procedures: a randomized study. Aust N Z J Obstet Gynaecol 2004:44:135-9

50. Morgan DM, Rogers MA, Huebner M, Wei JT, Delancey JO. Heterogeneity in anatomic outcome of sacrospinous ligament fixation for prolapse: a systematic review. Obstet Gynecol 2007:109:1424-33

51. Petri $E$, Ashok K. Sacrospinous vaginal fixation - current status. Acta Obstet Gynecol Scand 2011;90:429-36

52. Aigmueller T, Riss P, Dungl A, Bauer H. Long-term follow-up after vaginal sacrospinous fixation: patient satisfaction, anatomical results and quality of life. Urogynecol J Pelvic Floor Dysfunct 2008;19:965-9

53. DeLancey JO, Morgan DM, Fenner DE, Kearney R, Guire K, Miller JM, Hussain H, Umek W, Hsu $Y$, Ashton-Miller JA. Comparison of levator ani muscle defects and function in women with and without pelvic organ prolapse. Obstet Gynecol 2007:109:295-302

54. Zacche MM, Mukhopadhyay S, Giarenis I. Trends in prolapse surgery in England. Int Urogynecol J 2018;29(11):1689-95 


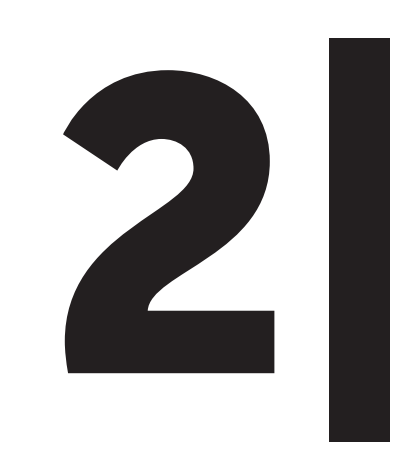

PRACTICE PATTERN VARIATION IN SURGICAL MANAGEMENT OF PELVIC ORGAN PROLAPSE AND URINARY INCONTINENCE IN THE NETHERLANDS

M.N. van IJsselmuiden

R.J. Detollenaere

M.Y. Kampen

M.K. Engberts

H.W.F. van Eijndhoven 


\section{ABSTRACT}

\section{INTRODUCTION}

Practice pattern variation (PPV) is the difference in care that cannot be explained by the underlying medical condition. The aim of this study was to describe PPV among Dutch gynecologists regarding treatment of pelvic organ prolapse (POP) and urinary incontinence (UI).

\section{MATERIALS AND METHODS}

PPV was calculated from data of healthcare declaration codes of 2010. Data were provided by Vektis and Kiwa Carity. PPV for POP and UI in general was calculated per hospital and per region. Furthermore, PPV for transvaginal mesh and surgical treatment of uterine descent was assessed.

\section{RESULTS}

PPV of surgical treatment for POP and UI in general was assessed for 91 hospitals. PPV for surgical treatment of uterine descent and transvaginal mesh placement was calculated for 88 hospitals. A high PPV per hospital and per region was found. In some hospitals, a hysterectomy was performed in all cases of uterovaginal prolapse, while in other hospitals, uterus-preserving techniques were mostly performed. A high PPV of transvaginal mesh placement was observed.

\section{CONCLUSION}

In the small country of The Netherlands, we found a high PPV in surgical management of POP and UI with respect to the choice for surgical treatment and the type of surgery. This finding might be due to the absence of clearly defined guidelines. Studies with respect to conservative versus surgical treatment and the type of surgery are of need to establish evidence-based guidelines.

\section{INTRODUCTION}

The choice for surgical treatment for pelvic organ prolapse (POP) in women mainly depends on the prolapse severity, symptoms, the general health of the woman, and the preference and capabilities of the surgeon [1]. Although there are many surgical techniques available, well-executed, randomized controlled trials to evaluate these different techniques are limited. Like POP, various treatment modalities for urinary incontinence (UI) have been described. In clinical practice, it is a convention that nonsurgical therapies, e.g., physiotherapy, mechanical devices, drugs, or neuromodulation, are applied first, because they usually carry a smaller risk of harm [2]. Therefore, surgery for treating $\mathrm{UI}$ is usually considered an option after failure of conservative therapy.

The availability of many treatment modalities can lead to variation in care between physicians and hospitals, the so called practice pattern variation (PPV). PPV is defined as the difference in care that cannot be explained by the underlying medical condition [3]. It can be caused by noncompliance to evidence-based practice standards, lack of evidence for optimal treatment, or by a difference in skills and resources. If patient characteristics, surgical capabilities, and indication for surgical interventions are equally spread among hospitals, there should be minimal PPV. PPV is considered to be a great problem in controlling medical costs [4].

In the literature, PPV has been reported in several medical conditions. Decades ago, regional PPV was already observed. In 1936, it was reported that tonsillectomy rates in Britain varied substantially between the different school districts [5]. In South Florida, USA, rates for surgery to treat degenerative diseases of hip, knee, and spine are highly variable among different hospital referral regions [6]. Furthermore, rates of coronary bypass surgery, prostatectomy, and many other major procedures varied at least four- to fivefold across different hospital referral regions [7]. In addition, PPV has been reported in gynecology. Recently, geographical variation in hysterectomy rates and surgical routes for hysterectomy (vaginal/abdominal) for benign diseases was reported in The Netherlands [8]. Little is known about PPV in POP and UI. However, variable surgical policy for treating uterine descent [9] and the wide range in types of surgery [10] might contribute to PPV.

The Netherlands is a small, developed country. With almost 17 million inhabitants, the country is densely populated. In 2010, the country contained a total of 92 hospitals and private clinics, with 958 practicing gynecologists. Due to its small size and good 
registration, The Netherlands is a very eligible country in which to explore practice patterns. Because of its size and the limited number of gynecologists (who are all member of the Dutch gynecologic society and are well organized in different working parties), PPV should be theoretically low.

The aim of this study was to identify and describe PPV among Dutch gynecologists regarding POP and UI treatment.

\section{MATERIALS AND METHODS}

In The Netherlands, health insurance is mandatory. All procedures performed by hospitals are invoiced from the health insurance companies using declaration codes. Each medical condition has a specific declaration code; POP and UI have a common code (G25). Furthermore, the type of treatment (conservative/surgical) must be stated in this declaration code. For various medical conditions, the companies Vektis and Kiwa Carity collect these declaration codes. In addition, Vektis analyzes these codes and calculates PPV. To assess PPV for POP and UI, we used data and calculations obtained from these organizations. The year 2010 was chosen because more recent data from both data sets (Kiwa Carity and Vektis) were not available when this study started.

\section{VEKTIS}

Vektis (www.vektis.nl) collects data from all healthcare insurance companies in The Netherlands. This independent organization analyses these data with respect to costs and quality of healthcare and calculates practice variation using the declaration codes. Data we obtained were corrected for age and social economic status. Information about social economic status was received from the Dutch Institute for Social Research, a government agency. Vektis did not distinguish between the different POP and UI procedures because they have a common code. Therefore, all surgical procedures for POP and UI were combine.

Data analysis was performed in two ways: per region and per hospital service area. The Netherlands is divided into 39 regions. A region is defined as a geographical district with the same zip/postal code. These data reflect the treatment of patients living in that region, regardless of where the care was actually delivered. A hospital service area is determined by assigning all patients to a hospital according to the decision tree shown in in Fig. 1. These data are hospital specific and reflect patients treated per hospital regardless of the region in which the patient lives.
Practice variation was defined as the number of patients who underwent POP or UI surgery per 100,000 women. Based on 2010 data, the practice variation was calculated both per region and per hospital. Furthermore, hospital and regional practice variations were pooled, and the ratio between conservative versus surgical treatment of POP and $\mathrm{UI}$ was assessed. Hospitals were divided into the categories of university, teaching. and nonteaching hospitals. All declaration codes with respect to POP and UI (G25) were used. One private clinic was excluded because $<20$ surgical procedures were performed.

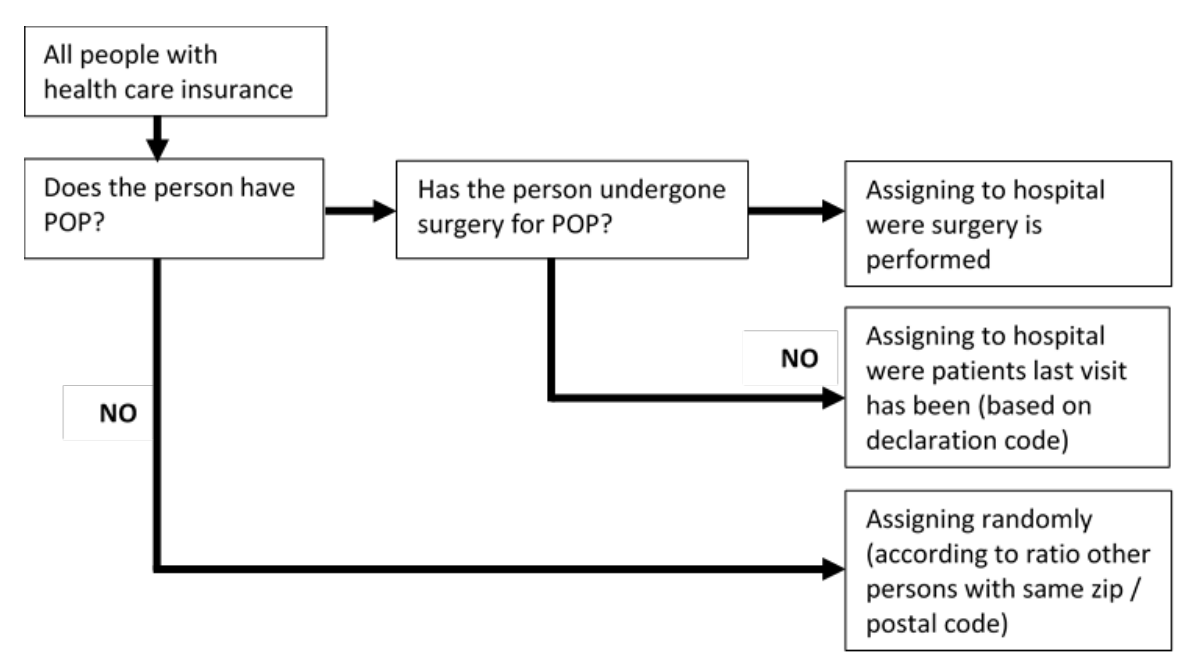

FIGURE 1. Creating hospital service area: assigning patients to a hospital

\section{KIWA CARITY}

Kiwa Carity (www.kiwacarity.nl) analyzes declaration codes to determine the quality and costs of healthcare, commissioned by the Dutch government and the healthcare branch. Since this company analyzes at the procedure level, it is possible to distinguish between different types of surgery for a specific condition. However, Kiwa Carity does not have access to data with respect to the total number of women with healthcare insurance and their demographic characteristics. Therefore, Kiwa Carity is not able to correct for age and social economic status. Data are based on the declaration of information system (DIS), where all declaration codes and medical procedures of the hospitals are recorded. Based on these data, we calculated the PPV of transvaginal mesh by dividing the total number of transvaginal mesh placements in 2010 per hospital by the total number of POP procedures. Furthermore, the ratio of uteruspreserving surgery versus vaginal hysterectomy for treating uterine descent was calculated. 
For both data sets, it was not possible to register more than one procedure per patient per year. In case of another surgical procedure for POP and/or UI in the same year, the same declaration code was used.

\section{STATISTICAL ANALYSIS}

Statistical analysis was performed with the Statistical Package for the Social Science 22.0 for Windows (IBM SPSS Statistics). Differences between hospital types were compared using one-way analysis of variance (ANOVA) for parametric variables, and Kruskal-Wallis test for nonparametric variables. An additional post hoc Bonferroni test was performed to assess the significance of each variable. A p value $<0.05$ was considered to be statistically significant.

\section{RESULTS}

PPV in general was assessed for 91 hospitals and for 88 hospitals with respect to treating uterine descent and the use of transvaginal mesh specifically. In 2010, more than 40,000 women were referred to a hospital because of POP and/or UI, and just over 15,000 surgical procedures were performed (Table 1). The number of patients per hospital varies depending on the size of the hospital service area. Differences in surgical rate between the different hospital types were not statistically significant $(p=0.11$ ).

Table 1. Total number of initial patients and procedures per practice type in 2010

\begin{tabular}{|c|c|c|c|}
\hline & $\begin{array}{l}\text { Number of initial G25- } \\
\text { patients (range) }\end{array}$ & $\begin{array}{l}\text { Number of POP and UI } \\
\text { procedures (range) }\end{array}$ & $\begin{array}{l}\text { Mean surgical rate } \\
\text { (\%) (procedures/G25) }\end{array}$ \\
\hline University hospital $(n=8)$ & $2.190(112-540)$ & $737 \quad(36-185)$ & 34 \\
\hline $\begin{array}{l}\text { Non-university teaching hospital } \\
(\mathrm{n}=28)\end{array}$ & $18.419(361-1.222)$ & $5.888(100-437)$ & 32 \\
\hline $\begin{array}{l}\text { Non-teaching hospital }(n=54) \\
\text { TOTAL }\end{array}$ & $\begin{array}{l}22.248(127-1.151) \\
42.857\end{array}$ & $\begin{array}{l}8.443(27-486) \\
15.068\end{array}$ & 38 \\
\hline
\end{tabular}

$\mathrm{G} 25$ = declaration code for POP and incontinence; $\mathrm{POP}$ = pelvic organ prolapse; $\mathrm{UI}=$ urinary incontinence

Figure 2 demonstrates the total number of patients who underwent POP and UI surgery per 100,000 women per hospital in 2010. This represents the PPV per hospital service area and varies between 75 and 412 procedures per 100,000 women (median 171). University hospitals seem to have a very high or very low surgical rate compared with other hospital types,. and nonteaching hospitals have a higher surgical rate than teaching hospitals.
Figure 3 shows the total number of patients who underwent POP and UI surgery per 100,000 women per region. This represents the practice variation per region and varies between 55 and 363 surgical procedures per 100,000 women (median 179). The figure implies a mixed pattern: the northeastern and western regions seem to have a higher surgical rates compared with central, southern, and eastern Netherlands.

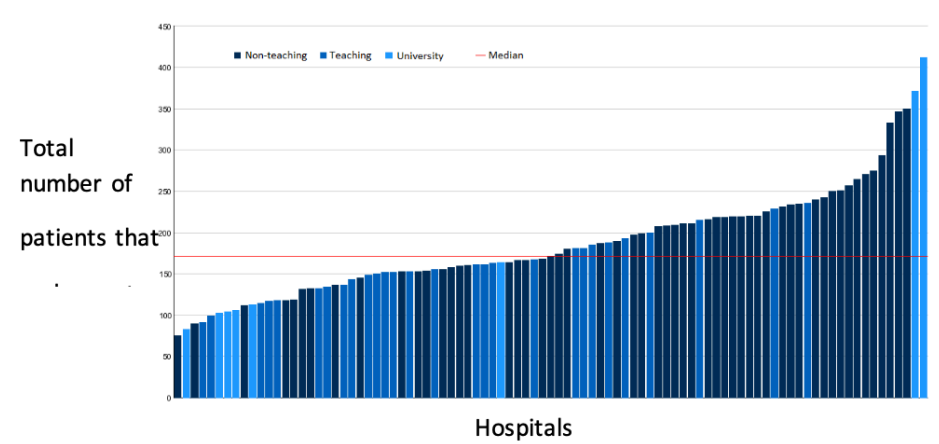

FIGURE 2. Practice variation per hospital: total number of patients that underwent pelvic organ prolapse (POP) or urinary incontinence (UI) surgery per 100.000 women per hospital service area in 2010

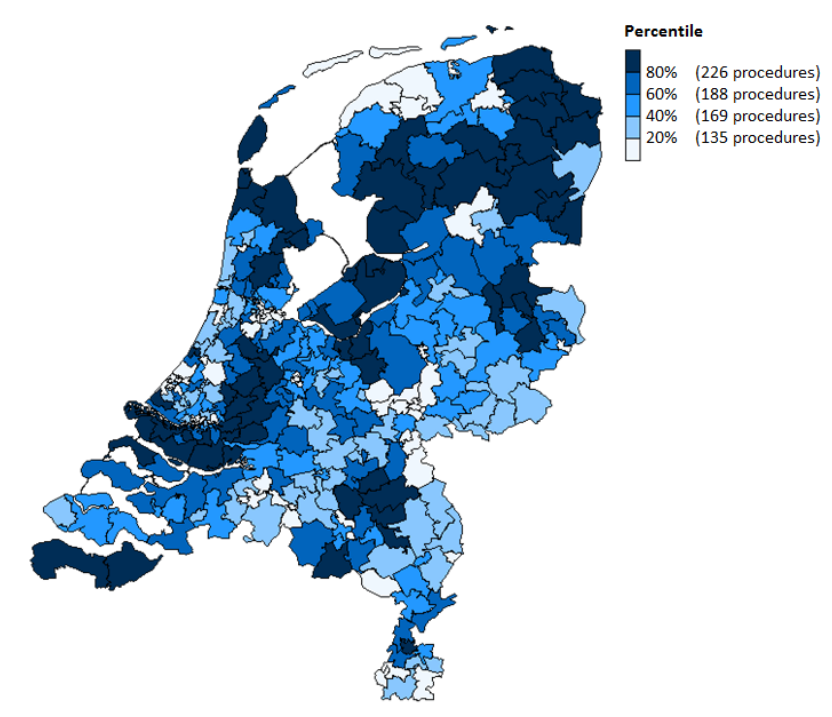

FIGURE 3. Practice variation per region: total number of patients that underwent pelvic organ prolaose (POP) or urinary incontinence (UI) surgery per 100.000 women per region in 2010. The minimum number of procedures per region was 55 per 100.000 women and the maximum number 363 per 100.000 women 
Figure 4 combines practice variation per hospital (see Fig. 2) with practice variation per region (see Fig. 3). It demonstrates surgical rate per hospital (displayed by the total length of the bar) and distinguishes the type of regions patients come from (displayed by the different colors of the bar). This figure indicates whether or not the hospital contributes to a high or low surgical rate in its region. If all patients who underwent POP or UI surgery in that hospital came from a region with a high surgical rate, it is likely that this hospital contributes to the high surgical rate in that region. In other words, when looking at a mostly dark-colored bar on the right side of the figure, it is plausible that this hospital might contribute to a high surgical rate in that particular region, while a mostly lightcolored bar at the left side might contribute to a low surgical rate in the region.

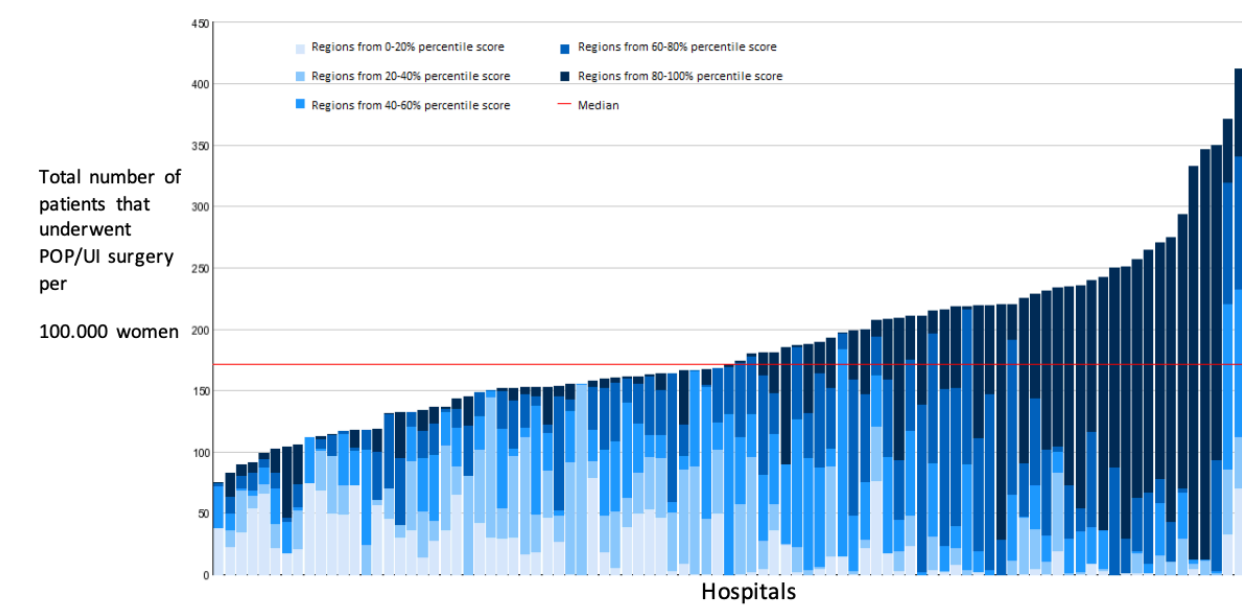

FIGURE 4. Practice variation for pelvic organ prolapse (POP) and urinary incontinence (UI) surgery per hospital combined with practice variation per region in 2010. The figure indicates whether the hospital contributes to a high or low surgical rate in that region. A mostly dark colored bar at the right side might contribute to the high surgical rate in a region, since all patient who underwent POP or UI surgery in that hospital come from a region with a high surgical rate. On the other hand, a mostly light colored bar a the left side might contribute to a low surgical rate in that region.

Figure 5 shows the ratio between conservative and surgical treatment for POP and UI per hospital. Again, hospitals are listed in the same order as in Figs. 2 and 4. Unlike the previous figures, in which POP and UI procedures were plotted against 100,000 women (the total population), this figure focuses on women with declaration code G25 who were referred to the hospital. It also shows that hospitals with a high surgical rate (right side of the figure) not only have a high surgical rate compared with the total population, but patients in that hospital will less often receive conservative treatment as well.

Figure 6 demonstrates the percentage of vaginal hysterectomy and uterus-preserving surgery for treating uterine descent. Uterus-preserving surgery is defined as vaginal sacrospinous hysteropexies and Manchester-Fothergill procedures. There is a high practice variation in treating uterine descent.

In some hospitals, a hysterectomy was performed in all cases of uterovaginal prolapse, while in others, patients were mainly treated with uterus-preserving surgery. This variation was observed in all types of hospitals. Nonteaching hospitals performed significantly more hysterectomies in relation to uterus-preserving techniques compared with teaching $(p=0.002)$ and university $(p=0.014)$ hospitals.

When looking at the practice variation with respect to transvaginal mesh placement, a high variation was observed (Fig. 7). In some hospitals, a transvaginal mesh was placed in 1:100 POP patients, while in other hospitals, it was placed in 44:100 POP patients. In 21 hospitals, it was not used at all. Hospitals with the highest number of transvaginal mesh placements per 100 POP patients were nonteaching hospitals. The differences in transvaginal mesh placement between hospital types were not statistically significant $(p=0.91)$.

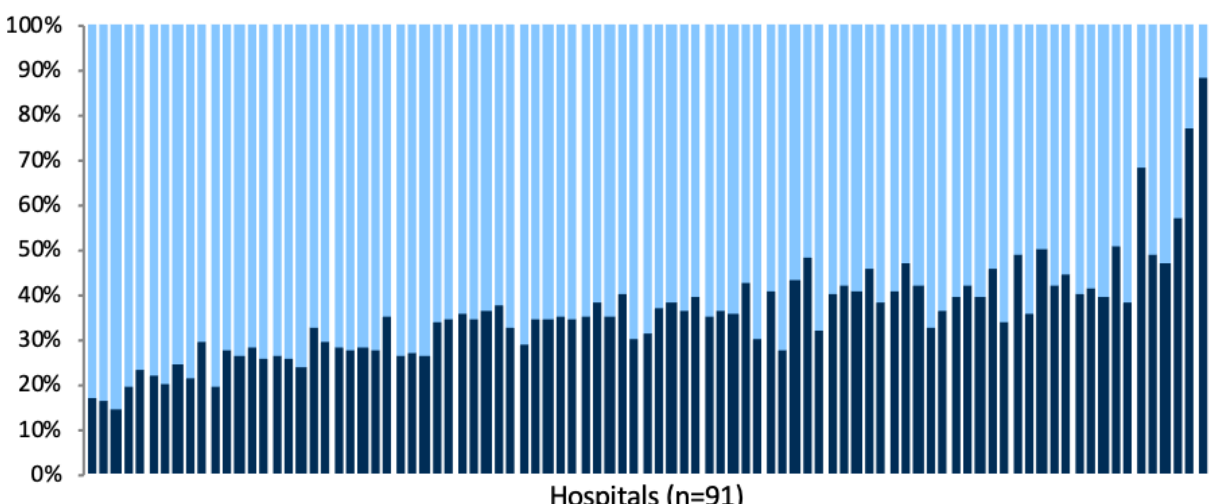

- Surgical treatment $\amalg$ Conservative treatm ent

FIGURE 5. Conservative versus surgical treatment for pelvic organ prolapse (POP) and urinary incontinence (UI) per hospital in 2010 (the hospitals are listed in the same order as figure 2 and 4). 


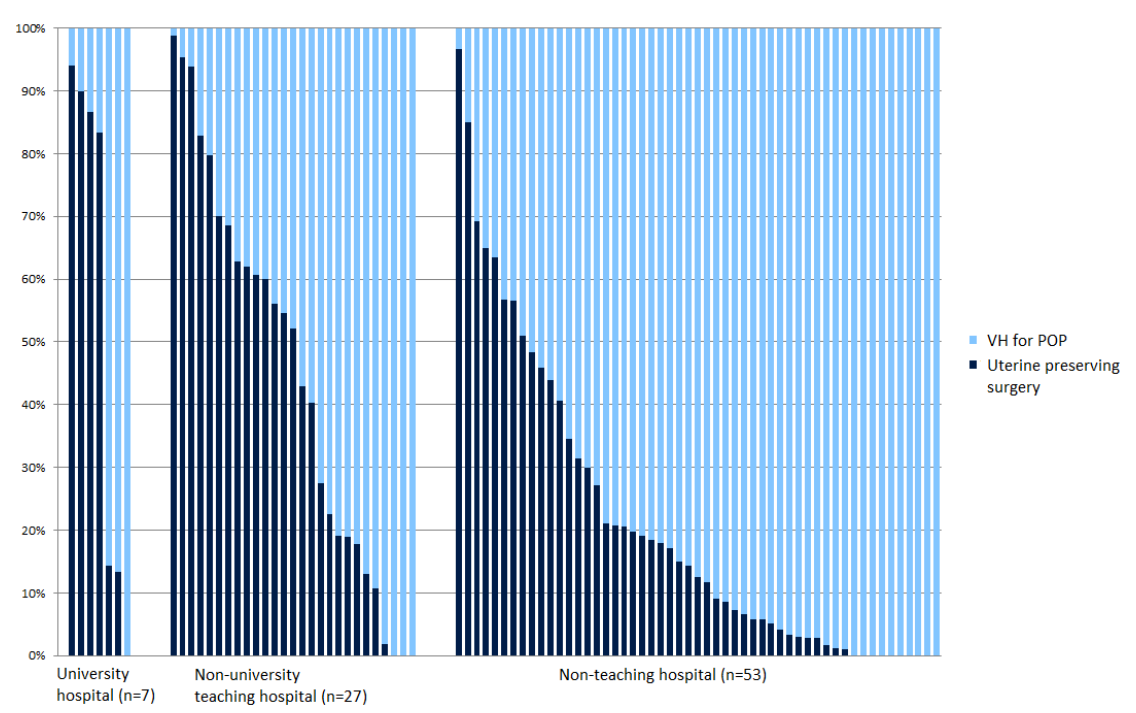

FIGURE 6. Practice variation in treatment of uterine descent: percentage of vaginal hysterectomy (VH) and uterine preserving surgery (sacrospinous hysteropexy and Manchester-Fothergill procedure) for treatment of uterovaginal prolapse per hospital.

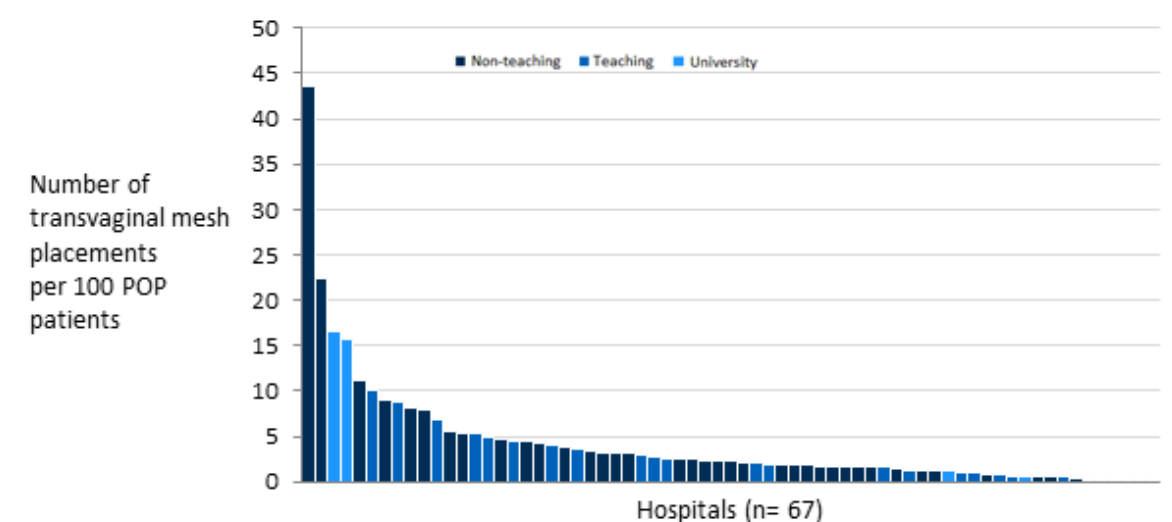

Figure 7. Practice variation of transvaginal mesh placement per hospital in 2010: number of transvaginal mesh placements per 100 patients referred to that hospital for pelvic organ prolapse (POP)

\section{DISCUSSION}

We assessed PPV for POP and UI surgery in The Netherlands. Our results demonstrate a high PPV with respect to treating POP and UI. These findings are inconsistent with our hypothesis that due to good cooperation and registration and the small size of The Netherlands, PPV should be low. We found a high PPV between regions. Because of the small size of the country, most regions include both rural and urban areas, and the different types of hospitals are equally spread throughout the country. Therefore, we believe that the regional differences cannot be explained by differences in population or hospital distribution. The surgical rate per region appears to be dominated by the treatment policy of specific hospitals in that specific region. Hospitals with a low surgical rate are actually responsible for the low surgical rate in their region. In addition, hospitals with a high surgical rate are responsible for the high surgical rate in their region. These hospitals seem to be mainly nonteaching hospitals (Figs. 2 and 4). In other words, the surgical rate per region appears to be caused by specific hospitals rather than being a mean of several hospitals.

The high PPV might imply over- and undertreatment. In case of overtreatment, the patient is exposed to the unnecessary risks of surgery. In case of undertreatment, the patient might not receive adequate treatment for the specific medical condition. However, since a gold standard for treating POP is still a matter of debate, it is difficult to conclude whether or not we observed over- or undertreatment.

The ratio between conservative and surgical POP and UI treatment varies per hospital. With regard to $\mathrm{UI}$, guidelines state that nonsurgical therapies are tried first ${ }^{[2,11,12]}$. In case of failure, surgical therapy is considered. A lack of adherence to the current guidelines for UI might contribute to the PPV. Contrary to UI, there is no consensus or guideline for treating POP conservatively or surgically. As a result, treatment depends mostly on the opinion of the gynecologist. Therefore, one can assume that the observed variation is mainly caused by variation in treating POP but not UI. However, because data were pooled, we were unable to differentiate between disorders.

Our results demonstrate a high PPV in the surgical treatment of uterine descent. Nonteaching hospitals perform significantly more hysterectomies in relation to uteruspreserving techniques compared with teaching and university hospitals. Worldwide, vaginal hysterectomy is the most frequently used treatment for patients with symptomatic uterovaginal prolapse ${ }^{[13,14]}$. However, uterus-preserving procedures are gaining interest, and many techniques have been described. Because well-executed randomized trials are limited, there is no consensus for either uterus-preserving surgery or vaginal hysterectomy. Therefore, our results seem to be a reflection of the ongoing discussion about the value of uterus-preserving techniques in case of uterine descent 
There is a high PPV with respect to transvaginal mesh placement. In recent years, transvaginal mesh has become matter of debate. In October 2008, the US Food and Drug Administration (FDA) issued a public health notification informing clinicians and patients of adverse events related to urogynecologic use of surgical mesh [15]. After this publication, the use of transvaginal mesh in the USA decreased from $32.1 \%$ in 2006 to $27.5 \%$ in 2010 [16]. In The Netherlands, the debate over safety and efficacy of vaginal mesh is ongoing. An important issue in this debate is the number of meshes placed per gynecologist. The high PPV and therefore limited experience of some gynecologists is one reason that recently formulated guidelines state that vaginal mesh placement is allowed only when a minimum of 20 procedures a year are performed.

In the literature, several factors contributing to PPV in general have been described. First, variations in patient characteristics and demand for surgery might contribute to PPV. Some regions might have a higher rate of surgical procedures because of greater demand for surgery. This correlation is more obvious in clinical disorders for which surgery is strongly recommended or needed, e.g., hip fracture. In the USA, regional rates of hip-fracture surgery in elderly people are almost linearly correlated with the regional incidence of hip fracture [7]. Second, the preference of the patient can contribute to PPV. For some clinical conditions, the decision to intervene should depend as much on patient preferences as on scientific evidence. The value of a surgical intervention depends on the tradeoff between risks and benefits of that intervention. In case of a large imbalance, the decision to intervene is distinct, and there might be some PPV. However, PPV becomes more pronounced when the tradeoff is a close call, and the patient's preference can become decisive [7]. Shared decision making is a collaborative process that allows patients and physicians to make healthcare decisions together, taking into account the best scientific evidence available as well as patient preference. Third, scientific uncertainty contributes to PPV [6]. In the absence of a professional consensus and guideline-based on outcomes, individual or small groups of clinicians can hold on to their own clinical rules of defining which patient needs surgery. This might result in the "surgical signature" of a region: rates for specific surgical procedures that are peculiar to a region sometimes differ substantially between surrounding regions $[4,6]$. Finally, surgical innovations and new technologies, such as vaginal mesh, can pronounce PPV by increasing the number of therapeutic alternatives available to clinicians. This variation can arise because of differences in clinicians' opinions as to whether the new intervention should replace the existing procedures and change the indications for surgery [7]

PPV is considered to be a great problem in controlling medical costs. In case of overtreatment, medical costs will be very high compared with undertreatment. Such variation is unfair, because people who live in a region with a low surgical rate (or are referred to a hospital with a low surgical rate) subsidize the care of those in highcost regions [3]. In the USA, the difference in lifetime Medicare (federal social health insurance) spending for a typical 65-year-old in Miami, FL, and in Minneapolis, MN, is $>\$ 50,000[4]$

A strength of this study is the completeness of the data sets. In The Netherlands, hospitals are required to use declaration codes to receive funding from healthcare insurance companies. Without a declaration code, it is not possible for insurance companies to invoice the procedure. Since the declaration code is compulsory, we obtained complete data sets. Another strength of this study is the correction for age and social economic status in case of PPV in general, making data more valid. Vektis determined whether age and social economic status were relevant in the total number of patients with UI and POP. For instance, if in a specific region live more older people compared with another region, differences in surgical rate are more likely explained by the differences in population statistics than by PPV. In their analysis, age and social economic status influenced the surgical rate, and therefore, data were corrected by Vektis for age and social economic status.

The study reported here has some limitations: Since UI and POP have the same declaration code, it is not possible to differentiate between these two conditions with respect to PPV in general. It is unknown whether the practice variation is caused by UI, POP, or both. Since clearly defined guidelines for $\mathrm{UI}$ are available in The Netherlands, and clear consensus on treating POP is lacking, we assume that the PPV is mostly caused by POP. In addition, a clearly defined diagnosis of POP (e.g., the compartment involved) cannot be obtained from the Vektis data set. However, since most types of prolapse can be treated surgically or conservatively, we believe that the PPV we found is applicable to treating POP in general. Furthermore, data derived from Vektis are based on 91 hospitals, and Kiwa Carity provided data obtained from only 88 hospitals. This difference is explained by the fact that Kiwa Carity did not include data from private clinics $(n=1)$, and two hospitals did not consent to Kiwa Carity using their data. With respect to the practice variation of uterine descent (Fig. 6), data of one university hospital is missing.

Ideally, PPV is very low, and the surgical rate for POP and UI does not differ between hospitals. However, in the small country of The Netherlands, we observed a high PPV in the surgical management of POP and UI. Furthermore, we observed a high variation in treating uterine descent and the placement of transvaginal mesh for POP. A patient's 
odds of undergoing surgery for POP and $\mathrm{UI}$ seems to depend more on where the patient lives and to which hospital she is referred than on clinical circumstances. The choice of treatment seems to depend mainly on the preference of the gynecologists. The absence of clearly defined guidelines regarding indications for conservative and surgical treatment of POP and the use of different types of surgical technique might contribute to the high PPV. With respect to UI, there might be a lack of adherence to the current guidelines for UI, which contributes to the PPV as well. To reduce PPV and prevent over- and undertreatment, studies with respect to conservative versus surgical treatment, and randomized controlled trials between different types of surgery, are necessary to establish such evidence-based guidelines.

\section{REFERENCES}

1. Maher C, Feiner B, Baessler K, Schmid C (2013) Surgical management of pelvic organ prolapse in women. Cochrane Database Syst Rev;: :CD004014

2. Lucas $M G$, Bedretdinova D, Bosch JLHR, Burkhard F, Cruz F, Nambiar AK, Nilsson CG, Ridder DJMK de, Tubaro A, Pickard RS (2014) Guidelines on urinary incontinence. Available at: http:// www.uroweb.org/gls/pdf/20\%20Urinary\%20 Incontinence_LR.pdf Accessed April 2014

3. Wennberg JE (2002) Unwarranted variations in healthcare delivery: implications for academic medical centers. BMJ;325(7370):961-964

4. Wennberg JE, Fisher ES, Skinner JS (2002) Geography and the debate over Medicare reform. Health Aff (Millwood):Supp Web Exclusives:W96-114.

5. Glover JA (2008) The incidence of tonsillectomy in school children. 1938. Int J Epidemiol;37:9-19

6. Weinstein JN, Bronner KK, Morgan TS, Wennberg JE (2004) Trends and geographical variations in major surgery for degenerative diseases of the hip, knee, and spine. Health Aff (Millwood); Supp Variation:VAR81-9

7. Birkmeyer JD, Reames BN, McCulloch P. Carr AJ, Campbell WB, Wennberg JE (2013) Understanding of regional variation in the use of surgery. Lancet;382:1121-9

8. Hanstede MM, Burger MJ, Timmermans A, Burger MP (2012) Regional and temporal variation in hysterectomy rates and surgical routes for benign diseases in the Netherlands. Acta Obstet Gynecol Scand; 91:220-25

9. Detollenaere RJ, Boon J den, Kluivers KB, Vierhout ME, Eijndhoven HWF van (2012) Surgical management of pelvic organ prolapse and uterine descent in the Netherlands. Int Urogynecol J:24:781-788

10. Yune JJ, Siddighi S (2013) Perceptions and practice patterns of general gynecologists regarding urogynecology and pelvic reconstructive surgery. Female Pelvic Med Reconstr Surg;19(4):225-9

11. Smith A, Bevan D, Douglas HR, James D (2013) Management of urinary incontinence in women: summary of updated NICE guidance. BMJ;347:55170

12. Dutch Society of Obstetrics \& Gynecology (NVOG) (2012) Guideline on urinary incontinence in women.http://nvog-documenten.nl/uploaded/ docs/Urine-incontinentie\%20bij\%20vrouwen/ nieuwe $\% 20$ versie\%20Definitieve\%20richtlijn $\% 20$ urine\%20incontinentie\%20bij\%20vrouwen.pdf Accessed February 2012.

13. Jha S, Moran P (2011) The UK national prolapse survey: 5 years on. Int urogynecol J 22:517-28

14. Vanspauwen R, Seman E, Dwyer P (2010) Survey of current management of prolapse in Australia and New Zealand. Aust N Z J Obstet Gynaecol 50:262-7

15. Food and Drug Administration (2011) FDA Safety Communication: UPDATE on Serious Complications Associated with Transvaginal Placement of Surgical Mesh for Pelvic Organ Prolapse.http://www.fda.gov/MedicalDevices/ Safety/AlertsandNotices/ucm262435.htm. Accessed 13 July 2011

16. Rogo-Gupta L, Rodriguez LV, Litwin MS, Herzog TJ, Neugut Al, Lu YS, Raz S, Hershman DL, Wright JD (2012) Trends in surgical mesh use for pelvic organ prolapse from 2000 to 2010. Obstet Gynecol 120(5):1105-15 


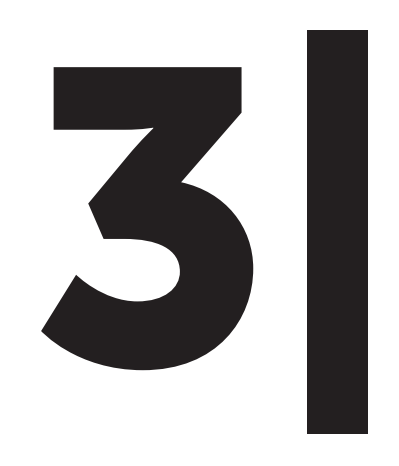

VARIATION IN THE PRACTICE OF LAPAROSCOPIC SACROHYSTEROPEXY AND LAPAROSCOPIC SACROCOLPOPEXY FOR THE TREATMENT OF PELVIC ORGAN PROLAPSE: A DUTCH SURVEY

M.N. van IJsselmuiden M.H. Kerkhof

R.P. Schellart

M.Y. Bongers

W.A. Spaans

H.W.F. van Eijndhoven 


\section{ABSTRACT}

\section{INTRODUCTION AND HYPOTHESIS}

Laparoscopic techniques for pelvic organ prolapse surgery using mesh are gaining interest. A standard approach or published guideline for the laparoscopic sacrohysteropexy (LSH) or laparoscopic sacrocolpopexy (LSC) is lacking. The purpose of this study is to assess the variation between Dutch gynecologists in executing LSH and LSC.

\section{METHODS}

A questionnaire was developed to evaluate the technique of LSH and LSC. All members of the Dutch Society for Gynecological Endoscopy and Minimally Invasive Surgery and the Dutch Society for Urogynecology were invited by email to participate in a webbased survey.

\section{RESULTS}

With 357 respondents, the response rate was $71 \%$. Of the respondents, a total of 49 gynecologists (13.7\%) perform LSH and/or LSC. Gynecologists who perform both procedures, use the same surgical technique for LSH and LSC. There are variations among gynecologists on several key points such as the level of dissection along the anterior and posterior walls of the vagina, the type of mesh used, the type of sutures used, the tension of the implanted mesh and reperitonealization of the mesh.

\section{CONCLUSIONS}

There is a high practice variation in LSH and LSC performed by a selected group of Dutch gynecologists. Different methods have been described in the literature and there is no consensus on how to perform these procedures. A well-designed prospective study or randomized controlled trial with regard to the specific parts of these procedures is needed to provide evidence for the best surgical technique. The outcomes of these studies will help to establish evidence-based guidelines.

\section{INTRODUCTION}

Pelvic organ prolapse (POP) is a common health problem. As life expectancy increases, more women will suffer from POP and will require surgery. The lifetime risk of surgery for POP by the age of 85 years is estimated to be $19 \%$ [1].

Traditionally, POP surgery has been performed via vaginal or abdominal approach. In an effort to improve outcomes in transvaginal reconstructive prolapse surgery, biologic and synthetic mesh devices have been introduced to complement, reinforce or replace native tissues. Subsequently, the use of mesh in prolapse surgery in the USA has increased from $7.9 \%$ in 2000 to a peak of $32.1 \%$ in 2006 [2].

Together with the growing use of transvaginal mesh, the number of reported adverse events has also increased. In October 2008, the Food and Drug Administration (FDA) issued a Public Health Notification to inform clinicians and patients of adverse events related to urogynecologic use of surgical mesh [3]. Following this notification, the FDA continued to monitor the outcomes of the use of surgical mesh. From 2008 to 2010, the most frequent complications reported to the FDA included vaginal mesh erosion, pain (including dyspareunia), infection, urinary problems, bleeding, and organ perforation [3]. After the publication of these safety advisories, the use of transvaginal mesh in the USA decreased slightly from $32.1 \%$ in 2006 to $27.5 \%$ in 2010 [2]. In the Netherlands, however, the use of vaginal mesh diminished dramatically after negative publicity in the media in 2012 .

The use of abdominal mesh appears to result in lower rates of complications compared to transvaginal POP surgery with vaginal mesh [4]. Two common types of abdominal prolapse repair are the sacrohysteropexy (in case of uterine descent) and the sacrocolpopexy of the vaginal vault after hysterectomy. In these procedures, uterus or vaginal vault is elevated by attaching the cervix or vaginal vault to the sacral promontory using a mesh $[5,6]$. Although the open abdominal procedures have been performed for many years, the laparoscopic approach of these procedures is relatively new $[7,8]$. In studies comparing abdominal sacrocolpopexy with the laparoscopic approach, these procedures have shown clinical equivalence [9], but laparoscopic sacrocolpopexy seems to be related to less procedure-related morbidity concerning blood loss, hospital stay and severe complications [10].

Although the laparoscopic approach is associated with good outcome and minimal morbidity, the use of rigid laparoscopic instruments can constraint suturing in and

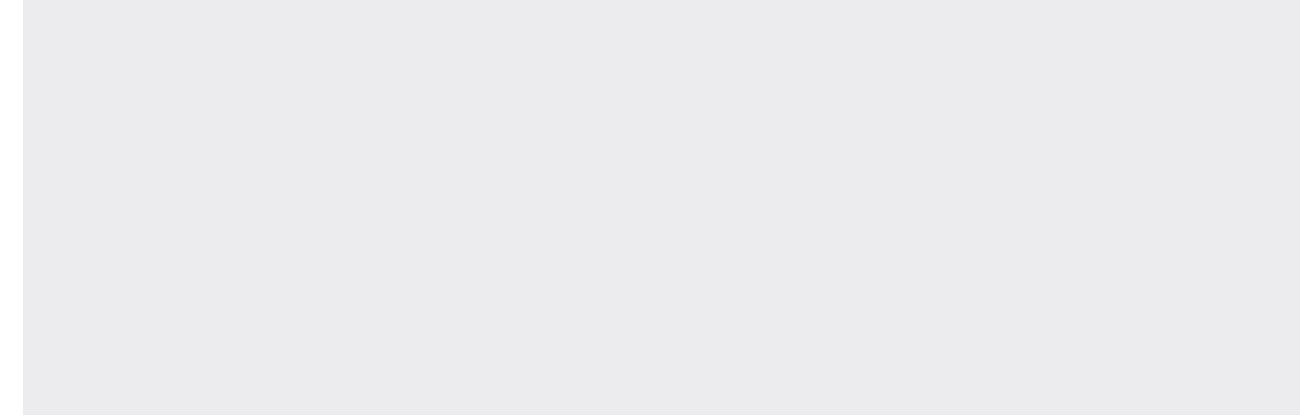


accessing to the deep pelvis. Because of the steep learning curve, laparoscopic prolapse repair is generally reserved for expert laparoscopists [11]. In literature, different surgical methods for laparoscopic sacrohysteropexy (LSH) and laparoscopic sacrocolpopexy (LSC) have been described and there is no consensus how to perform these laparoscopic procedures $[7,9,12,13]$.

Because of the negative publicity surrounding transvaginal mesh and advantages of laparoscopic surgery, we expect that the numbers of laparoscopic POP surgery will increase. In the Netherlands, a standard approach or published guideline for LSH or LSC is lacking. In addition, a standard laparoscopic mesh kit, as known in transvaginal mesh procedures, is not available. Therefore, analysis of the different laparoscopic POP procedures will be of utmost importance to be able to evaluate outcomes of these procedures. The purpose of this study is to assess the variation among gynecologists in executing laparoscopic POP procedures. The knowledge of these practice variation will help to design future protocols for these procedures.

\section{MATERIALS AND METHODS}

We conducted a cross-sectional study in the Netherlands, consisting of a questionnaire. In January 2014, all members of the Dutch Society for Gynecological Endoscopy and Minimally Invasive Surgery and the Dutch Society for Urogynecology $(n=503)$ were invited by email to participate in a web-based survey. A total of $52.5 \%$ of all practicing gynecologists in the Netherlands, is member of one or both of these Societies. A reminder email was sent 2 weeks after the initial survey invitation, and after 5 weeks the identical questionnaire was sent by post to the non-responders.

\section{QUESTIONNAIRE}

The questionnaire addressed the following topics: characteristics of the hospital and the gynecologist, laparoscopic characteristics, preoperative treatment of patients and specifications of the procedure. The survey was self-developed. Prior to the start of the study, we performed a pilot study among 5 gynecologists. Based on the recommendations of the pilot participants, the questionnaire was revised. All questionnaires were treated anonymously. No financial compensation was given.

\section{STATISTICS}

Descriptive statistics were performed using SPSS 22.0 (SPSS Statistics UK, SPSS Inc, Chicago, IL, USA). Results were expressed as mean and range for continuous variables.
In case of ordinal variables, proportions were calculated.

\section{RESULTS}

The questionnaire was sent to the 503 members of Dutch Society for Gynecological Endoscopy and Minimally Invasive Surgery and the Dutch Society for Urogynecology. With 357 respondents, the response rate was $71 \%$. Of the respondents, a total of 49 gynecologists (13.7\%) reported that they perform LSH and/or LSC. The characteristics of these gynecologists are shown in table 1.

TABLE 1. Characteristics of the respondents performing laparoscopic sacrohysteropexy and/or laparoscopic sacrocolpopexy

\begin{tabular}{|c|c|}
\hline Characteristic & $n(\%), N=49$ \\
\hline \multicolumn{2}{|l|}{ Practice type } \\
\hline Academic hospital & 7 (14) \\
\hline Non-academic teaching hospital & $34(69)$ \\
\hline Non-teaching hospital & $8(16)$ \\
\hline \multicolumn{2}{|c|}{ Experience in laparoscopic procedures (years) } \\
\hline $2-5$ & $5(10)$ \\
\hline $5-10$ & 11 (22) \\
\hline$>10$ & $33(67)$ \\
\hline \multicolumn{2}{|l|}{ Performing laparoscopic sacrohysteropexy } \\
\hline Yes & $39(80)$ \\
\hline No & $10(20)$ \\
\hline \multicolumn{2}{|c|}{$\begin{array}{l}\text { Number of sacrohysteropexies performed in the past } 12 \text { months } \\
\text { as first surgeon }\end{array}$} \\
\hline$<10$ & $23(59)$ \\
\hline $10-30$ & $15(38)$ \\
\hline $31-50$ & 1 (3) \\
\hline \multicolumn{2}{|l|}{ as second surgeon } \\
\hline$<10$ & 30 (77) \\
\hline $10-30$ & $9(23)$ \\
\hline \multicolumn{2}{|c|}{ Change in number of sacrohysteropexies compared with 12 months ago } \\
\hline Increased & $20(51)$ \\
\hline Decreased & $4(10)$ \\
\hline No difference & $15(38)$ \\
\hline \multicolumn{2}{|l|}{ Performing laparoscopic sacrocolpopexy } \\
\hline Yes & 45 (92) \\
\hline No & $4(8)$ \\
\hline \multicolumn{2}{|c|}{$\begin{array}{l}\text { Number of sacrocolpopexies performed in the past } 12 \text { months } \\
\text { as first surgeon }\end{array}$} \\
\hline$<10$ & $22(49)$ \\
\hline $10-30$ & $22(49)$ \\
\hline $31-50$ & $1(2)$ \\
\hline \multicolumn{2}{|l|}{ as second surgeon } \\
\hline$<10$ & $29(64)$ \\
\hline $10-30$ & $16(36)$ \\
\hline
\end{tabular}


TABLE 1. (continued)

\begin{tabular}{lc}
\hline Characteristic & $\mathbf{n}(\%), \mathbf{N}=49$ \\
\hline $\begin{array}{l}\text { Change in number of sacrocolpopexies compared with 12 months ago } \\
\text { Increased }\end{array}$ & $23(51)$ \\
Decreased & $3(7)$ \\
No difference & $19(42)$ \\
& \\
Preference of gynecologist to perform laparoscopic supracervical hysterectomy prior & \\
to sacrocolpopexy & $18(40)$ \\
Yes & $27(60)$ \\
No, only sacrocolpopexy in case of posthysterectomy vaginal vault & \\
prolapse & \\
\hline
\end{tabular}

\section{PREOPERATIVE CHARACTERISTICS}

Table 2 shows the results of the questions related to laparoscopic surgery in general. The composition of the surgery team differs, depending on experience training and the usage of a robot. Bowel preparation prior to surgery is not a standard procedure. Methods used for bowel preparation are emptying the rectum by flushing with a syringe filled with water or povidone, moviprep, enemata and bisacodyl drops. Of the respondents, $90 \%$ routinely administer antibiotic prophylaxis. Antibiotics are given 30 minutes prior to the procedure $(n=28,64 \%)$ or during administration of anesthesia $(n=16,36 \%)$. Cefazolin in combination with metronidazole is the most used prophylaxis $(n=21,43 \%)$, followed by cefuroxime and metronidazole $(n=9,18 \%)$, amoxicillin/ clavulanic acid $(n=6,12 \%)$ and cefazoline alone $(n=5,13 \%)$. One respondent stated to administer intravenous amoxicillin/clavulanic acid for 3 days postoperative, after an initial administration of cefazolin/metronidazole perioperatively.

TABLE 2. General characteristics of laparoscopy

\begin{tabular}{lc}
\hline Laparoscopic surgery (general) & $\mathbf{n}(\%), \mathbf{N}=\mathbf{4 9}$ \\
\hline Team composition & \\
One gynecologist, one instrument nurse and one scrub nurse & $4(8)$ \\
Two gynecologists and one instrument nurse & $19(39)$ \\
One gynecologist, one general surgeon and one instrument nurse & $3(6)$ \\
One gynecologist, one resident and one instrument nurse & $9(18)$ \\
Other (e.g. with robot) & $14(29)$ \\
Robot-assisted approach & \\
Yes & $10(20)$ \\
No & $39(80)$ \\
Pre-operative bowel preparation & $5(10)$ \\
Yes & $44(90)$ \\
No & \\
Routine administration of antibiotics peri-operatively & $44(90)$ \\
Yes & $5(10)$ \\
No & \\
\hline
\end{tabular}

Table 3 shows the specific characteristics for LSH and LSC. The mean number of trocars inserted is the same for both procedures and depends on the use of a robot: in case of a robot-assisted approach all gynecologists insert 5 trocars; when the robot is not used the mean number of trocars inserted is 4 (LSH range 3-6; LSC range 3-5). Mostly, trocars are placed umbilical, suprapubic and symmetric left and right in the iliac region.

\section{DISSECTION}

Of the respondents, the majority starts the surgery with dissection of the peritoneum and the promontory (LSH $n=29(59 \%) ; \operatorname{LSC} n=32(71 \%))$. The depth of the anterior and posterior dissection of the cervix or vaginal vault differs (table 3). In contrast to posterior dissection, anterior dissection of the cervix or vaginal vault is not routinely performed by all gynecologists: the mesh is only attached to the posterior cervix or vaginal vault in $18 \%$ (LSH) and $4 \%$ (LSC). One respondent mentioned another extent for dissection of the posterior site: until the musculus levator ani on the right site and until the rectal fold on the left site. With respect to the anterior site, dissection until the balloon of the urinary catheter or until $3-4 \mathrm{~cm}$ of the anterior vaginal wall were mentioned as other extents for dissection.

\section{MESH CHARACTERISTICS AND ATTACHMENT}

Most gynecologists use polypropylene meshes (table 3). In addition, the majority of the respondents use $\mathrm{Y}$-shaped meshes (prefabricated or prepared during surgery). Most respondents attach the mesh both anterior and posterior of the cervix or vaginal vault (double mesh). None of the gynecologists attach the mesh only to the anterior site. The mean number of sutures for attaching the mesh to the anterior cervix or vaginal vault is 5 (LSH range $1->5$; LSC range $3->5$ ). To attach the mesh to the posterior cervix or vaginal vault, a mean of 5 sutures are used (LSH range 1- $>5$; LSC range 3 $>5$ ). Different types of sutures for attaching the mesh to the cervix or vaginal vault are used, with a preference for the non-absorbable multifilament (LSH 46\%, LSC 49\%). In addition, the material for attachment of the mesh to the promontory varies: mean number of sutures/tackers/staples used is 3 (LSH range 1-5; LSC range 3-5). The mesh is attached to the promontory with slight tension or tension free. To assess the tension, several techniques are used: visually, vaginal examination and/or vaginal manipulator. 
TABLE 3. Characteristics of laparoscopic sacrohysteropexy and laparoscopic sacrocolpopexy Characteristics

acement of trocars

Umbilical, suprapubic, left and right (symmetrically)

Umbilical, two left, one right

Umbilical, wo right one le

\%), $\mathrm{N}=45$

Other

$\begin{array}{cc}27(69) & 30(67) \\ 2(5) & 3(7) \\ 1(3) & 2(4) \\ 9(23) & 10(22) \\ & \\ 11(28) & 12(27) \\ 11(28) & 13(29) \\ 17(44) & 20(44)\end{array}$

Yes, always

No, never

Only in case of poor visibility

20 (44)

Dissection

Technique of dissection of peritoneum

Entire peritoneum (from promontory to pouch of Douglas) unneling of caudal peritoneum

$31(79) \quad 34(76)$

$8(21) \quad 11(24)$

xtension of posterior dissection of cervix/vaginal vault

Up to the level of the cervix/vault and maximum the upper part of the

posterior vaginal wall

Up to halfway the posterior vaginal wall

Dissection of musculus levator ani on both sides

$$
\text { Depending on the situation }
$$

$11(28) \quad 8(18)$

$11(28) \quad 13(29)$

$14(36) \quad 20(44)$

$\begin{array}{ll}3(8) & 4(9) \\ 2(5) & 3(7)\end{array}$

Extension of the anterior dissection of cervix/vaginal vault

Up to the level of the cervix/vault and maximum $1-2 \mathrm{~cm}$ of anterior

vaginal wall

Up to $1-2 \mathrm{~cm}$ under the bladder

As deep down as possible

Other

Depending on the situation
Not performed

Other

Introduction mesh and attachment

Mesh

Attachment

Only posterior
Both anterior and posterior

Other

Depending on the situatio

Characteristics $^{\star}$$$
\text { Other }
$$

Polypropylene mesh

Mersilene graft

during surgery)

Prefabricated Y-shaped mesh

Single leaf

Individually cut to fit/adjusted

Type of sutures for attaching mesh to cervix/vault

Absorbable monofilament

Non-absorbable monofilament

Absorbable multifilament

Non-absorbable multifilament
$12(31) \quad 9(20)$

$10(26) \quad 18(40)$

$10(26) \quad 11(24)$

$\begin{array}{cc}7(18) & 7(15) \\ 2(5) & 3(7)\end{array}$

$3(8) \quad 0(0)$

2(5) $\quad 4(9)$

$\begin{array}{rr}7(18) & 2(4) \\ 27(69) & 37(82)\end{array}$

$5(13)-6(13)$

$\begin{array}{ll}3(8) & 3(7) \\ 2(5) & 3(7)\end{array}$

$38(97) \quad 44(98)$

1(3) $\quad 1(2)$

$\begin{array}{lr}3(8) & 3(7) \\ 13(33) & 25(56)\end{array}$

$\begin{array}{ll}9(23) & 3(7)\end{array}$

$19(49) \quad 20(44)$

$\begin{array}{rr}3(8) & 4(9) \\ 13(33) & 12(27)\end{array}$

$\begin{array}{ll}5(13) & 7(16) \\ 18(46) & 22(49)\end{array}$ $\begin{array}{ll}13(33) & 12(27) \\ 5(13) & 7(16)\end{array}$
TABLE 3. (continued)

\begin{tabular}{lcc}
\hline Characteristics & $\begin{array}{c}\text { LSH } \\
\text { (\%), N=39 }\end{array}$ & $\begin{array}{c}\text { LSC } \\
(\%), \mathbf{N}=45\end{array}$ \\
\hline $\begin{array}{c}\text { Attachment of mesh to promontory* } \\
\text { Sutures }\end{array}$ & $9(23)$ & $9(20)$ \\
$\quad$ Non-absorbable monofilament & $3(8)$ & $2(4)$ \\
Absorbable multifilament & $1(3)$ & $1(2)$ \\
Non-absorbable multifilament & $5(13)$ & $6(13)$ \\
Tackers & $23(59)$ & $28(62)$ \\
Staples & $10(26)$ & $13(29)$ \\
How much tension is used to attach mesh to promontory & & \\
Tension free & $22(56)$ & $20(44)$ \\
Slight tension & $16(41)$ & $24(53)$ \\
Tension is not important & $1(3)$ & $1(2)$ \\
Assessment of tension & $10(26)$ & $13(29)$ \\
Visually & $12(31)$ & $13(29)$ \\
Vaginal examination & $14(36)$ & $15(33)$ \\
Vaginal manipulator (uterus / vaginal manipulator) & $3(8)$ & $4(9)$ \\
Other (combination of above) & $1(3)$ & $0(0)$ \\
Routinely performance of cystoscopy afterwards & $38(97)$ & $45(100)$ \\
Yes & $10(26)$ & $7(16)$ \\
No & $3(8)$ & $1(2)$ \\
Performance of concomitant colporrhaphy & $5(13)$ & $5(11)$ \\
Yes $\quad$ Prior to laparoscopy & $2(5)$ & $1(2)$ \\
$\quad$ After laparoscopy & $29(74)$ & $38(84)$ \\
\hline$\quad$ Other & &
\end{tabular}

LSH = laparoscopic sacrohysteropexy, LSC = laparoscopic sacrocolpopexy

\section{REPERITONEALIZATION AND CONCOMITANT COLPORRHAPHY}

The majority of the respondents close the peritoneum over the mesh (LSH 92\%, LSC 91\%) using absorbable sutures, Quill/V-lock or staples. Cystoscopy after the surgery is not routinely performed: only one gynecologist performs a cystoscopy after a LSH. Furthermore, concomitant colporrhaphy in case of prolapse of the anterior and/or posterior vaginal wall is not standard and is performed by the minority of the respondents (LSH 26\%, LSC 15\%).

\section{DISCUSSION}

We performed a nationwide survey to assess practice variation of LSH and LSC. To our knowledge, this is the first study that focuses on differences in surgical techniques of LSC and LSH in POP surgery. Our results demonstrate a high practice variation of these procedures. 
Only $13.7 \%(n=49)$ of the responding Dutch gynecologists perform LSH and/or LSC. This small number can probably be explained by the complexity and the long learning curve of these procedures. Therefore, laparoscopic prolapse repair is generally supposed to be the domain of expert laparoscopists [11]. Despite the small number of gynecologists performing this type of surgery, $51 \%$ of the respondents observed an increase in the number of LSH and LSC in the last 12 months. This might be explained by the diminished use of transvaginal mesh after the publication of safety advisories and the negative publicity [2], which possibly led to a growing interest in laparoscopic POP surgery. Gynecologists who perform both procedures, use the same surgical technique for LSH and LSC. However, we found variation in surgical technique among gynecologists.

Most gynecologists do not administer bowel preparation. If bowel preparation is performed, different methods are used. In the literature, there is no evidence regarding bowel preparation in laparoscopic prolapse surgery. In gynecological laparoscopy in general, bowel preparation improves surgical view and bowel handling, but the benefit is thought to be small [14]. One randomized controlled trial demonstrated that mechanical bowel preparation is well-tolerated, but it does not influence the surgical field visualization for laparoscopic hysterectomy [15].

Most Dutch gynecologists routinely administer perioperative antibiotics, which is in line with most large series describing laparoscopic prolapse surgery $[9,16]$. There are no specific studies on the use of antibiotic prophylaxis at the time of mesh reconstructive surgery for prolapse [17]. As a consequence, there is no evidence if, and if so, which antibiotics should be given to prevent postoperative infection.

The majority of the respondents prefer to dissect the entire peritoneum (from promontory to pouch of Douglas), while some gynecologists create a tunnel under the caudal peritoneum to avoid later suturing. Again, there is no evidence in literature which technique is better.

In contrast to posterior dissection, an anterior dissection of the cervix or vaginal vault is not routinely performed by the respondents. As related to the dissection of cervix or vaginal vault, the mesh is mostly attached to the anterior and posterior site of the cervix or vaginal vault (double mesh). None of the respondents attach the mesh to the anterior site only. However, in a minority the mesh is attached to the posterior site only. In a few large series of LSC reported in literature, the majority of authors systematically place a double mesh, which is in line with the systematical treatment of the three compartments of the pelvis (anterior, apical and posterior) [18]. A double mesh is associated with a reduction in anatomical recurrence, the posterior mesh demonstrated to have a high effectiveness in correcting and preventing a posterior compartment prolapse [18]. However, systematic placement of a posterior mesh is responsible for specific complications, like rectal injury, postoperative constipation and postoperative perianal pain [18]. Therefore, literature suggests to reserve the posterior mesh (or double mesh) for cure of enterocele or rectocele. In cases without prolapse of the posterior vaginal wall, an anterior mesh only would be sufficient. However, these conclusions are based on retrospective data. A well-designed prospective study or randomized controlled trial with respect to this item is of need to provide evidence for the best technique for mesh attachment.

Since a standard laparoscopic mesh kit does not exist, our results demonstrate practice variation in the type and shape of the mesh. The majority of the gynecologists (97\%) use a mesh made of polypropylene, some gynecologists use a prefabricated Y-shaped mesh, while others prefer to prepare a Y-shaped mesh during surgery. Mostly, nonabsorbable sutures are used to attach the mesh to the cervix/vault and tackers to attach the mesh to the promontory. In literature, we found no studies concerning surgical outcome and the material for attaching the mesh to the cervix or vaginal vault in case of sacrocolpopexy and sacrohysteropexy (open or laparoscopic). One study compares non-absorbable sutures with tackers to attach the mesh to the prevertebral ligament of the promontory. The main advantage of the use of staplers is the ease. Spondylodiscitis is a rare complication, the risk is enhanced by the deeper infiltration of the staples in the intervertebral discs. Fixation of the mesh to the promontory by using sutures is much stronger than by staples [19].

One of the most critical steps of these procedures is to determine the appropriate amount of tension to suspend the uterus or vaginal vault sufficiently without causing pain, de novo stress incontinence or de novo dyspareunia [20]. In Dutch practice, the mesh is usually attached tension free or with slight tension. In the literature, several studies reported to attach the mesh tension free [12] or even with moderate tension [13]. However, there is no standard with respect to the amount of tension and the best way to assess this.

After the uterus or vault is suspended, most respondents cover the mesh with peritoneum in an attempt to avoid bowel entrapment and internal hernia [20]. However, in a study of 128 women undergoing sacrocolpopexy without burial of mesh by peritoneal closure, no bowel complications were reported [21]. Nevertheless, after 
partial closure of the peritoneum over the mesh, adhesions between the mesh and loops of small bowel were noticed at subsequent laparoscopies [13].

Only one gynecologist routinely performs a cystoscopy after LSH. Intraoperative cystoscopy can be performed to detect bladder injury. The exact incidence of mesh misplacement in the bladder or sutures penetrating the bladder are unknown. In literature, the removal of intravesical mesh following LSC has been described in several case studies [22,23]. However, diagnosis was made months after the initial surgery. Therefore, it is unknown if these mesh protrusions were due to cystotomies during the initial surgery or to erosion or migration [23]. Furthermore, cystoscopy can detect ureteral injury by assessing urine efflux from the ureteral orifices [24]. The overall incidence of ureteral injury is estimated to be $<1-2 \%$ in gynecological laparoscopy [24]. Potential disadvantages of performing universal intraoperative cystoscopy are increased operative time, procedure costs and incidence of minor complications such as urinary tract infection and bladder trauma. Furthermore, cystoscopy will not detect all injuries, particularly those caused by thermal damage, which may take several weeks to develop [25]. Cystoscopy could lead to overdiagnosis and unnecessary intervention, since some urinary tract injuries are asymptomatic and resolve spontaneously [25]. Because the potential disadvantages of intraoperative cystoscopy and the possibility to identify the ureter during laparoscopy, literature suggests to perform intraoperative cystoscopy only when urinary tract injury is suspected during gynecologic laparoscopy $[24,25]$. However, no recommendations for intraoperative cystoscopy with respect to POP surgery with abdominal mesh could be found in literature.

Concomitant colporrhaphy in case of prolapse of the anterior or posterior vaginal wall after LSH and LSC is only performed by a minority of the gynecologists. The majority of these respondents prefer to perform anterior or posterior colporrhaphy after the laparoscopy. In literature, it is thought that elevating the vaginal vault or cervix may correct anterior and posterior vaginal wall prolapse. Furthermore, deep attachment of the anterior and posterior mesh can repair anterior and posterior vaginal wall prolapses as well [26]. However, there is a risk of enterotomy or cystotomy [20]. In a retrospective cohort study comparing outcomes after abdominal sacrocolpopexy with and without concomitant paravaginal anterior wall repair, no statistical differences in recurrence rates of cystoceles were detected [27]. With respect to the posterior wall, abdominal sacrocolpopexy without concomitant posterior repair sufficiently restores posterior vaginal wall support in most women at one year follow up [28]. Furthermore, concomitant posterior repair after robotic sacrocolpopexy did not significantly affect bowel symptoms [29].
The strength of this study is the high response rate to this questionnaire $(71 \%)$. There are also some limitations of the study protocol. A questionnaire is always a simplification of the reality. It is impossible to question all minor details of the surgical technique, which will be influenced by various details of the surgical anatomy and expertise of the surgeon. Furthermore, the questionnaire is anonymous and we did not ask for the hospital where the respondent is currently working. Therefore, it might be possible that a part of respondents work in the same hospital using the same local protocol. These factors might influence the data and its interpretation. Another limitation of this study is the small number of respondents who perform LSH and LSC $(n=49)$. Membership of the Dutch Society of Obstetrics \& Gynecology (NVOG) is compulsory for all Dutch practicing gynecologists. The NVOG has several subdivisions including the Dutch Society for Gynecological Endoscopy and Minimally Invasive Surgery or the Dutch Society for Urogynecology. All gynecologists who perform laparoscopic prolapse surgery are most likely member of one or both societies. Therefore, we are quite certain that we have reached all the relevant gynecologists. Because laparoscopic prolapse repair is reserved for expert gynecologists, this procedure is only performed by the minority of Dutch gynecologists. That might explain that, in a small country as the Netherlands with a total of 958 gynecologists, there will be a small number of gynecologists who perform LSH and LSC. However, despite this small group, we were able to demonstrate great variations in the practice of these techniques.

Laparoscopic POP surgery seems to be on the increase, probably because of the long-term complications of the vaginal mesh [3]. However, a standard protocol for laparoscopic prolapse repair is lacking. While laparoscopic application of these procedures might be relatively new, the abdominal approach has been performed for many years. Surgical techniques for this open procedure have been described in literature $[5,6,9,10]$. Several studies have compared the open abdominal sacrocolpopexy to the laparoscopic sacrocolpopexy. In these studies, the technique for open procedure is identical to the laparoscopic procedure on several key points $[9,10]$. Despite the experience in abdominal sacrohysteropexy and sacrocolpopexy, our study demonstrates that gynecologists differ in performing LSH and LSC on several key points like level of dissection along the anterior and posterior wall of the vagina, type of mesh used, type of sutures used, tension of the implanted mesh and reperitonealization of the mesh. To monitor outcome and long-term complications of laparoscopic prolapse repair, consensus of how to perform these procedures seems mandatory. It might not be possible to achieve complete standardization, since some points depend on the preference of the gynecologist. In addition, it can be discussed if standardization of the whole procedure would be relevant, as some variables probably 
do not contribute to the surgical outcome (like trocar placement and cystoscopy afterwards). In some key points of the procedure, standardization would be realizable and beneficial in comparing surgical outcomes. In our opinion, these are extent of dissection, attachment of the mesh to cervix or vaginal vault (anterior and/or posterior) and type of sutures for attachment of the mesh. The varying surgical techniques and the absence of accepted guidelines make comparing of surgical outcomes difficult. Unfortunately, this is not unique for the laparoscopic prolapse repair. The same concerns consist about almost every urogynecological procedure, such as anterior colporrhaphy [30]. There is no consensus on antibiotics, use of suture material and so on.

We conclude that there are variations in the practice of LSH and LSC performed by a selected group of gynecologists. This variation is a reflection of the absence of a clearly defined guideline. However, conducting a guideline is complex since there is no evidence in the specific parts of the procedure. A well-designed prospective study or randomized controlled trial with regard to the specific parts of these procedures is needed to provide evidence for the best surgical technique. The outcomes of these studies will help establish an evidence-based guideline. This may reduce the variations in practice and enable comparison of surgical outcomes.

\section{REFERENCES}

1. Smith FJ, Holman CD, Moorin RE, Tsokos N (2010) Life time risk of undergoing surgery for pelvic organ prolapse. Obstet Gynecol 116(5):1096-100

2. Rogo-Gupta L, Rodriguez LV, Litwin MS, Herzog TJ, Neugut Al, Lu YS, Raz S, Hershman DL, Wright JD (2012) Trends in surgical mesh use for pelvic organ prolapse from 2000 to 2010. Obstet Gynecol 120(5):1105-15

3. Food and Drug Administration (2011) FDA Safety Communication: UPDATE on Serious Complications Associated with Transvaginal Placement of Surgical Mesh for Pelvic Organ Prolapse. http://www.fda.gov/MedicalDevices/ Safety/AlertsandNotices/ucm262435. htm. Accessed 13 July 2011

4. Maher C, Feiner B, Baessler K, Schmid C (2013) Surgical management of pelvic organ prolapse in women. Cochrane Database of Systematic Reviews DOI: 10.1002/14651858.CD004014.pub5

5. Leron E, Stanton SL (2001) Sacrohysteropexy with synthetic mesh for the management of uterovaginal prolapse. BJOG 108(6):629-33

6. Nygaard IE, McCreery R, Brubaker L, Connolly A, Cundiff G, Weber AM, Zyczynski H (2004) Abdominal sacrocolpopexy: a comprehensive review. Obstet Gynecol 104(4):805-23

7. Rosenblatt PL, Chelmow D, Ferzandi TR (2008) Laparoscopic sacrocervicopexy for the treatment of uterine prolapse: a retrospective case series report. J Minim Invasive Gynecol 15(3):268-72

8. Nezhat CH, Nezhat F, Nezhat C (1994) Laparoscopic sacrocolpopexy for vaginal vault prolapse. Obstet Gynecol 84(5): 885-8

9. Freeman RM, Pantazis $K$, Thomson A, Frappell J, Bombieri L, Moran P, Slack M, Scott P, Waterfield M (2013) A randomised controlled trial of abdominal versus laparoscopic sacrocolpopexy for the treatment of post-hysterectomy vaginal vault prolapse: LAS study. Int Urogynecol J 24(3):37784

10. Coolen AL, van Oudheusden AM, van Eijndhoven HW, van der Heijden TP, Stokmans RA, Mol BW, Bongers MY (2013) A comparison of complications between open abdominal sacrocolpopexy and laparoscopic sacrocolpopexy for the treatment of vault prolapse. Obstet Gynecol Int doi:10.1155/2013/528636

11. Pollard ME, Eilber KS, Anger JT (2013) Abdominal approaches to pelvic prolapse repairs. Curr Opin Urol 23:306-11

12. Sarlos D, Kots L, Rye G, Schaer G (2014) Longterm follow-up of laparoscopic sacrocolpopexy. Int Urogynecol J 25(9):1207-12

13. Rahmanou P, Price N, Jackson S (2014) Laparoscopic hysteropexy: a novel technique for uterine preservation. Int Urogynecol J 25(1):13940

14. Won H, Maley P, Salim S, Rao A, Campbell NT, Abbott JA (2013) Surgical and patient outcomes using mechanical bowel preparation before laparoscopic gynecological surgery: a randomized controlled trial. Obstet Gynecol 121(3):538-46

15. Siedhoff MT, Clark LH, Hobbs KA, Findley AD, Moulder JK, Garrett JM (2014) Mechanical bowel preparation before laparoscopic hysterectomy: a randomized controlled trial. Obstet Gynecol 123:562-7

16. Ramanah R, Ballester M, Chereau E, Bui C, Rouzier $R$, Daraï E (2012) Anorectal symptoms before and after laparoscopic sacrocolpoperineopexy for pelvic organ prolapse. Int Urogynecol J 
23(6):779-83

17. De Tayrac R, Sentilhes $L$ (2013) Complications of pelvic organ prolapse surgery and methods of prevention. Int Urogynecol J 24(11):1859-72

18. Antiphon $P$, Elard $S$, Benyoussef A, Fofana $M$, Yiou R, Gettman M, Hoznek A, Vordos D, Chopin DK, Abbou CC. Laparoscopic promontory sacral colpopexy: is the posterior, recto-vaginal mesh mandatory? Eur Urol 45(5):655-61

19. Boukerrou M, Orazi G, Nayama M, Boodhun R, Crépin G, Cosson M (2003) Promontofixation procedure: use of non-absorbable sutures or tackers. J Gynecol Obstet Biol Reprod 32(6):5248

20. Parkes IL, Shveiky (2014). Sacrocolpopexy for the treatment of vaginal apical prolapse: evidence based surgery. J Minim Invasive Gynecol 21(4):546-57

21. Elneil $S$, Cutner AS, Remy $M$, Leather AT, Toozs-Hobson P, Wise B (2005) Abdominal sacrocolpopexy for vault prolapse without burial of mesh: a case series. BJOG 112(4):486-9

22. Frenkl TL, Rackley RR, Vasavada SP, Goldman HB (2008) Management of iatrogenic foreign bodies of the bladder and urethra following pelvic floor surgery. Neurourol Urodyn 27(6):491-5

23. Maher C, Feiner B (2011) Laparoscopic removal of intravesical mesh following pelvic organ prolapse mesh surgery. Int Urogynecol J 22(12):1593-5

24. Manchouri E, Cohen SL, Sandberg EM, Kibel AS, Einarsson J (2012) Ureteral injury in laparoscopic gynecologic surgery. Rev Obstet Gynecol 5(2):106-11

25. Sandberg EM, Cohen SL, Hurwitz S, Einarsson JI (2012) Utility of cystoscopy during hysterectomy Obstet Gynecol 120(6):1363-70.

26. Dimitri S, Thomas A, Gabriel S (2014) Laparoscopic sacrocolpopexy with deep attachment of anterior and posterior mesh. Int Urogynecol J 25(11):1591-2

27. Shippey SH, Quiroz LH, Sanses TV, Knoepp LR, Cundiff GW, Handa VL (2010) Anatomic outcomes of abdominal sacrocolpopexy with or without paravaginal repair. Int Urogynecol J 21(3):279-83 28. Guiahi M, Kenton K, Brubaker L (2008) Sacrocolpopexy without concomitant posterior repair improves posterior compartment defects. Int Urogynecol J Pelvic Floor Dysfunct 19(9):1267-

29. Crane AK, Geller EJ, Matthews CA (2013) Outlet constipation 1 year after robotic sacrocolpopexy with and without concomitant posterior repair. South Med J 106(7):409-14

30. Lensen EJM, Stoutjesdijk JA, Withagen MIJ, Kluivers KB, Vierhout ME (2011) Technique of anterior colporrhaphy: a Dutch evaluation. Int Urogynecol J 22:557-561 
DUTCH WOMEN'S ATTITUDES TOWARDS HYSTERECTOMY AND UTERUS PRESERVATION IN SURGICAL TREATMENT OF PELVIC ORGAN PROLAPSE

M.N. van IJsselmuiden R.J. Detollenaere M.B.E. Gerritse K.B. Kluivers M.Y. Bongers H.W.F. van Eijndhoven 


\section{ABSTRACT}

\section{OBJECTIVE}

To investigate Dutch women's attitudes and preferences towards hysterectomy or uterus preservation in surgical treatment of pelvic organ prolapse.

\section{STUDY DESIGN}

Women's attitude was assessed by a structured questionnaire in one university hospital and one non-university teaching hospital in the Netherlands. Between December 2013 and November 2014, 102 women referred with prolapse complaints, without previous prolapse surgery, responded to the questionnaire received by mail prior to gynaecological consultation. Main outcome was the preference for uterus preserving surgery versus hysterectomy. Furthermore we studied the impact of uterus preservation and hysterectomy on body image and sexual function and the importance of treatment success, risk of urinary incontinence after surgery, complication risk, recovery time, length of hospital stay, costs and the risk of developing endometrial cancer.

\section{RESULTS}

Assuming that functional and anatomical outcomes after hysterectomy and uterus preserving surgery were equal, more women expressed preference for uterus preservation (43\%, 44 out of 102 women) compared to hysterectomy $(27 \%, 27$ out of 102 women). The majority of women expected a similar improvement in sexuality and body image after the two treatment modalities. Treatment success, risk for urinary incontinence after surgery and complication risk were the most important factors. Taken the future risk of endometrial cancer into account, $18 \%$ of the women preferred hysterectomy because of this risk.

\section{CONCLUSIONS}

This study demonstrated that women referred with prolapse complaints have a preference for uterus preservation in case outcomes after both interventions are expected to be equal. The majority of women expected that body image and sexual function would equally improve after both interventions

\section{INTRODUCTION}

Different surgical techniques are available to treat pelvic organ prolapse (POP). Whether or not the uterus should be preserved in case of uterine prolapse is still a matter of debate. Well-executed randomized controlled trials to evaluate these therapies are limited and based on current literature no clear superiority in favour of uterus preserving surgery or hysterectomy is known [1-3].

In the Netherlands, a trend towards uterus preserving surgery was found during the last decade [4]. A similar change was observed in Taiwan and the US [5,6]. A possible explanation for this phenomenon could be that gynaecologists have become more familiar with the different operative uterus sparing techniques. Also women's belief and opinion about the treatment of uterine prolapse may have changed. In literature it is often stated that more women want to preserve the uterus in case of surgical management of uterine prolapse. However, little is known on women's attitude, preference and belief with respect to uterus preservation or hysterectomy in surgical management.

The aim of this study was to explore attitude towards hysterectomy and uterus preservation in Dutch women referred with POP complaints. Patient's perception and belief on the impact of uterus preservation and hysterectomy on body image and sexual function were also studied. Furthermore we studied the importance of treatment success, risk of urinary incontinence after surgery, complication risk, recovery time, length of hospital stay, costs and the risk of developing endometrial cancer when choosing one of the treatment options.

\section{MATERIALS AND METHODS}

Women's attitude towards hysterectomy or preservation was assessed by a structured preference questionnaire in a cross-sectional study during one year. The study took place in a large teaching hospital (Isala Zwolle) and a university hospital (Radboud University Nijmegen Medical Centre) in The Netherlands from December 2013 to November 2014. Both hospitals obtained approval for this study from the central medical ethical committee of the Isala (March 8, 2012, registration number 12.0326)

All women aged 18 years or older, referred with POP complaints by their general practitioner (GP), without previous prolapse surgery or hysterectomy, could participate 
in the study. Eligible patients were identified and selected based on the referral letters by the investigators ( $M v I J, M G$ and KK). Prior to the scheduled gynaecological consultation, eligible patients received written and oral study information and were asked to participate. Written informed consent was obtained from all participants. Demographic data were collected and prior to the consultation all patients were requested to complete two questionnaires: 1) a self-developed questionnaire and 2) a standardized validated quality of life and disease-specific questionnaire as recommend by the Dutch Urogynaecological Society including the Urogenital Distress Inventory (UDI) [7-9].

Primary objective of the study was to assess women's attitudes towards uterus preservation versus vaginal hysterectomy in surgical treatment of uterine prolapse. Secondary objectives were women's perception and opinion on: 1. Impact of uterus preserving surgery and vaginal hysterectomy on body image and sexuality, 2. Importance of treatment success, risk of urinary incontinence after surgery, complication risk, recovery time, length of hospital stay, costs and sexual functioning after surgery in choosing a surgical POP procedure and 3. Influence of future risk to develop endometrial cancer on the preferred treatment.

In the self-developed questionnaire the aim of the study was explained and information on POP and surgical treatment was given. First, women were questioned whether they preferred uterus preserving surgery or vaginal hysterectomy assuming equivalence. Furthermore we counselled women based on what was known from literature at that time based on the latest reviews regarding this subject: 1. Complication risk during and short after surgery (bleeding, infection, damage to the bladder or bowel and problems with micturition) appears to be equal after both interventions [10]; 2 . Some women need repeat surgery because of recurrent prolapse. It is not clear whether this is more frequent after uterus preservation or hysterectomy $[1,2,10]$; 3 . It is possible that surgery relieves symptoms but also other complaints can occur. A small number of women will suffer from urinary incontinence after surgery for POP. Removal of the uterus is possibly more often associated with urinary incontinence in comparison to uterus preservation, but this need further investigation [11]; 4. Some uterus preserving procedures are associated with a shorter hospital stay (about a day shorter) and a faster recovery after surgery $[10,12,13]$. Women were asked whether this information affected their preferred surgical approach. Subsequently, there were specific questions about the influence of the type of surgical approach on the expected change in improvement of body image and sexual functioning after surgery. Furthermore, women rated on a 5 -point Likert scale ( $1=$ not important, $5=$ very important) the importance of the following factors when choosing surgery: 1. treatment success, 2. risk of urinary incontinence after surgery, 3. post-operative recovery, 4. costs, 5. length of hospital stay, 6. complications, and 7. sexual functioning after surgery. Finally, patients were explained that with uterus preservation there is a small risk of getting endometrial cancer. Information was given that in The Netherlands every year approximately 1900 women develop endometrial cancer and this type of cancer is usually discovered at an early stage due to blood loss. The 5-year overall survival of this early stage disease is $95 \%$. Women were asked whether the risk of endometrial cancer influenced their preferred treatment.

A pilot study was performed among 15 women referred with POP complaints. These women completed the preference questionnaire under supervision of one of the study investigators (RJD) and several modifications were made to increase the readability and to clarify some questions. The results of this pilot study were not used in the final analysis. The POP-Q score and UDI questionnaire scores were collected from the medical record to describe baseline characteristics.

All statistical analyses were performed in SPSS for Windows version 22.0.0.1 (SPSS Inc., Chicago, IL, USA). Patient characteristics were summarized using descriptive statistics for continuous variables presented with medians, means, range and standard deviations as appropriate. Differences were compared using one-way analysis of variance (ANOVA).

\section{RESULTS}

During one year, a total of 200 women were asked to participate of which 102 women (52\%) actually responded and filled out the questionnaire. In 30 women (29\%) UDI scores were missing. Table 1 shows the baseline characteristics. Of the participating women, 36 (35\%) were aged 18 to 50 years and 66 (65\%) over 50 years of age. Furthermore, 32 (33\%) appeared to have a uterine prolapse POP-Q stage two or higher assessed by POP-Q examination. Anterior vaginal wall prolapse stage two or higher was found in $68 \%$ of the women and $24 \%$ had posterior vaginal wall prolapse stage two or higher.

Outcomes on preference for hysterectomy or uterus preservation assuming equivalence are shown in table 2. In case of equal outcome $43 \%$ (44 out of 102) preferred uterus preservation, $27 \%$ (27 out of 102) preferred hysterectomy and 30\% (31 out of 102) had no preference. 
TABLE 1. Baseline and anatomical characteristics

\section{Characters}

Age, years $\mathrm{n}=102$

Parity

Body mass index, $\mathrm{kg} / \mathrm{m}^{2}$

Postmenopausal

Highest educational level:

Primary / secondary school

High school

Bachelor, master or academic degree

VAS general health $(0-100)$

UDI subscale prolapse*

Overall POP-Q stage prolapse"

Data are presented as mean (SD) or number (percentage) as appropriate. Percentages were calculated using

non-missing data. UDI: Urogenital Distress inventory; VAS: visual analogue scale score;

UDI. $0=$ no symptoms or not bothersome and $100=$ most bothersome symptoms.

"Evaluated by Pelvic Organ Prolapse Quantification (POP-Q) examination.

TABLE 2. Preference for hysterectomy or uterus-preserving surgery assuming equal outcome

\begin{tabular}{lc}
\hline & Number of women (\%) \\
\hline Uterus preservation & $44(43 \%)$ \\
Hysterectomy & $27(27 \%)$ \\
No preference & $31(30 \%)$ \\
\hline
\end{tabular}

Table 3 demonstrates the relation between the women's preference and their characteristics. In the hysterectomy preference group, the percentage of postmenopausal women is higher as compared to the uterus preservation preference group (not significant). No significant differences in preoperative bladder and prolapse symptoms were found between the preference groups.

TABLE 3. Characteristics by treatment preference

\begin{tabular}{|c|c|c|c|}
\hline & $\begin{array}{l}\text { Uterus preservation } \\
\qquad(n=44)\end{array}$ & $\begin{array}{l}\text { Hysterectomy } \\
(n=27)\end{array}$ & $\begin{array}{c}\text { No preference } \\
(n=31)\end{array}$ \\
\hline Postmenopausal status & $24(55 \%)$ & $17(63 \%)$ & $24(77 \%)$ \\
\hline \multicolumn{4}{|c|}{ Bladder and prolapse symptoms (UDI)* } \\
\hline Obstructive micturition & $29.2(26.7) n=28$ & $33.3(24.7) n=21$ & $18.8(23.7) n=23$ \\
\hline Overactive bladder & $25.5(23.0) n=27$ & $23.2(21.1) n=22$ & $25.9(24.1) n=24$ \\
\hline Urinary incontinence & $23.2(24.1) n=28$ & $30.8(27.7) n=20$ & $32.6(28.0) n=24$ \\
\hline Discomfort/pain & $30.3(24.9) n=28$ & $38.3(24.8) n=20$ & $25.4(20.0) n=23$ \\
\hline Genital prolapse & $40.1(33.7) n=27$ & $25.4(29.6) n=21$ & $37.5(29.2) n=24$ \\
\hline
\end{tabular}

In table 4 the results of counselling are demonstrated. Statistically significant more women preferred uterus preservation $(65 \%)$ compared to hysterectomy $(13 \%$, $p=0.001$ ). Of women who preferred uterus preservation $96 \%$ opted for the same treatment after counselling. However, of the women who preferred hysterectomy, $50 \%$ changed their mind after counselling and preferred uterus preservation instead. Among the women who had no preference, $45 \%$ preferred uterus preservation after written counselling.

Several reasons were given to sustain the choice of treatment. Most common reasons to choose for hysterectomy were: to avoid problems in the future (43\%), menstrual disorders $(17 \%)$ and the feeling that the uterus has become unnecessary $(13 \%)$. Most frequently mentioned reasons for uterus preservation were: less invasive procedure $(22 \%)$, the wish to stay as complete as possible $(22 \%)$, not unnecessarily remove a healthy organ (22\%). Only one woman had preference for uterus preservation because of future childbearing.

TABLE 4. Preference for hysterectomy or uterus-preserving surgery assuming equal outcome and crossover of the preference after counselling.

\begin{tabular}{lcccc}
\hline \multicolumn{4}{c}{ Q1. Preference in case of equal outcome } \\
& Uterus preservation & Hysterectomy* & No preference & Total \\
\hline Q2. Preference after counselling* & & & & \\
Uterus preservation & $42(96 \%)$ & $10(36 \%)$ & $14(45 \%)$ & $66(65 \%)$ \\
Hysterectomy & $0(0 \%)$ & $13(50 \%)$ & $0(0 \%)$ & $13(13 \%)$ \\
No preference & $2(4 \%)$ & $3(12 \%)$ & $17(55 \%)$ & $22(22 \%)$ \\
\hline Data are presented as number (percentage). & & &
\end{tabular}
* Preference after counselling in one patient was missing with preference for hysterectomy in case of equal

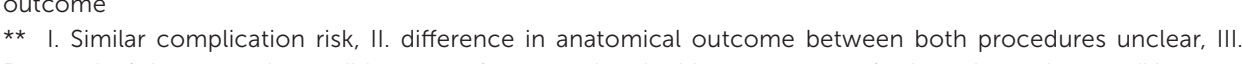
Removal of the uterus is possibly more often associated with occurrence of urinary incontinence, IV. uterus preserving procedures are associated with shorter hospital stay and faster post-operative recovery ${ }^{1.29 .9-12 !}$.

Table 5 shows outcomes on the improvement of body image and sexuality. The majority of women $(77 \%)$ expected that the improvement in body image would be the same after both interventions. Regarding sexuality, also $79 \%$ expected that both interventions would equally improve sexuality.

On a 5 -point Likert scale, treatment success $(4.5 \pm 0.7)$, risk of urinary incontinence after surgery $(4.5 \pm 0.8)$, complication risk $(4.4 \pm 0.7)$ and postoperative recovery (4.0 \pm 1.0 ) were the most important factors for the preference of a surgical intervention. 
In addition, sexual functioning after surgery was also regarded to be important (3.8 $\pm 1.1)$. Length of hospital stay $(2.9 \pm 1.1)$ and costs $(3.0 \pm 1.1)$ were found to be less important.

TABLE 5. Improvement in body image and sexuality

\begin{tabular}{lc}
\hline & No of women (\%) \\
\hline Body image & $79(77 \%)$ \\
Equally improvement in body image & $18(18 \%)$ \\
Uterus preservation more improves body image & $4(4 \%)$ \\
Hysterectomy more improves body image & $1(1 \%)$ \\
Missing & \\
Sexuality & $81(79 \%)$ \\
Equally improvement sexuality & $13(13 \%)$ \\
Uterus preservation more improves sexuality & $4(4 \%)$ \\
Hysterectomy more improves sexuality & $4(4 \%)$ \\
Missing &
\end{tabular}

Data are presented as number (percentage).

With respect to the future risk of endometrial cancer, $18 \%$ answered they preferred hysterectomy because of this risk, $52 \%$ would not necessarily choose hysterectomy and $30 \%$ did not know. Excluding women without a specific preference and taking age into account, more women aged under 50 years $(47.8 \%)$ preferred hysterectomy because of the future risk of endometrial cancer compared to women aged above 50 years $(14.6 \%, p=0.003)$.

\section{COMMENT}

This study analysed women's attitude towards hysterectomy and uterus preserving surgery in treatment of POP. In case of equal outcomes after these procedures, more women preferred uterus preservation (43\%) compared to hysterectomy $(27 \%)$. After counselling based on current knowledge from literature, the percentage of women preferring uterus preservation increased to $65 \%$. Our findings suggest that preoperative bladder and prolapse symptoms do not influence the preference. In the hysterectomy preference group, the percentage postmenopausal women is higher as compared to the uterus preservation preference group suggesting that having a menstrual cycle or having bleeding disorders does not influence the choice for hysterectomy over uterus preservation.
In the US, a preference assessment in women with POP symptoms reported that a higher proportion of women with prolapse symptoms preferred uterine preservation, instead of hysterectomy [14]. Assuming outcomes were equal between hysterectomy and uterine preservation, $36 \%$ of the women preferred uterine preservation; $20 \%$ of the women preferred hysterectomy, and $44 \%$ of the women had no clear preference. Overall, this is in line with our findings. Geographic region, education level, and belief that the uterus is important 'for a sense of self' were predictors of preference for uterine preservation. Another study with respect to patients' attitude toward the uterus and its role in surgery found that women had relatively neutral attitudes on the uterus being beneficial to sexuality or femininity [15]. The majority of respondents disagreed that the uterus was important for body image and sexuality. This is in line with our results; since approximately $80 \%$ of the women reported that the effect of uterus preservation and hysterectomy would be the same on sexuality and body image. A study among predominately Hispanic women concluded that $32 \%$ would choose to preserve their uterus at the time of prolapse repair [16].

In our study women completed the questionnaire before gynaecological consultation and examination. Therefore our results could not be influenced by the preference of the gynaecologist, or information on their actual prolapse. On the other hand, the fact that women did not really know whether or not they had an uterine prolapse or indication for surgery may have introduced bias into the study as their prolapse complaints could also be caused by anterior and/or posterior vaginal wall prolapse. Only one third of the respondents had an uterine prolapse POP-Q stage two or higher assessed by POP-Q examination. It is known that pelvic floor symptoms and prolapse complaints have a weak correlation with prolapse in a specific compartment or severity of the prolapse [17]. Therefore, we believe that this population was representative for this study. Another limitation was the relatively low response rate, with only half of the patients giving approval. The most important reason to decline participating was the bother to fill out the questionnaire. One could argue that the counselling statements encouraged women to choose for uterus preservation. The study would have been more complete if we had also used counter-factual statements providing advantages for hysterectomy over uterine preservation. The lack of providing such statements might be regarded as a limitation. The statements used for counselling were based on the best available evidence at the moment of project development. Furthermore, we did not ask the participants if their family was complete or if there was still a wish for childbearing. However, only one woman preferred uterus preservation because of future childbearing. Therefore, we assume that most participants had complete families. 
We have used a written, non-validated questionnaire, which was send to women prior to gynaecological consultation. Advantage of this type of survey is that it allows women to fill it out at their own convenience. Furthermore it is relatively inexpensive and little time consuming to health care providers, which makes it possible to send it to a large number of women with the possibility to analyse the results more objectively than other types of preference survey. Disadvantages of non-validated written questionnaires are low response rates and a level of subjectivity in the answers since people may read differently into each question and therefore reply based on their own interpretation. We tried to avoid this issue by piloting the questionnaire among women before the trial started.

In a previous study we found a trend toward more uterus preservation among gynaecologists in the Netherlands with large variation in treatment of uterine prolapse [4]. Better understanding of women's thoughts and preference on surgical treatment of uterine prolapse makes that more specific information can be given prior to surgery. This is valuable in the shared-decision making process prior to surgery. Women appointed treatment success, risk on urinary incontinence, complication risk and recovery time as important factors in the choice of a surgical procedure. In addition it seems that counselling had a significant effect on the preferred treatment. Caregivers must be aware of these points and evidence based information should be given prior to surgery. However, shared decision making is a delicate process. Not only the characteristics of the procedure should be discussed with the patient, but also the risk of future malignancy.

Data from randomised trials on the outcomes of different prolapse operations are sparse. Recently, we published the results from the SAVE U trial, comparing uterus preserving sacrospinous hysteropexy with vaginal hysterectomy with suspension of the uterosacral ligaments [3]. This RCT demonstrated that both anatomical and functional outcome were equal for these operative techniques. Although vaginal hysterectomy used to be the most performed operation in case of uterine prolapse ${ }^{[4,18,19]}$, these results provide us an alternative comparable treatment. Better understanding of women's preferences on uterus preservation makes it possible to come to a process of shared-decision making with respect for women's attitudes in case of POP surgery.

\section{CONCLUSIONS}

This study demonstrated that women referred with prolapse complaints have a preference for uterus preservation in case the outcomes after both interventions are equal. The majority of women expected that body image and sexual function would equally improve after both interventions. 


\section{REFERENCES}

1. Maher C, Feiner B, Baessler K, Schmid C. Surgical management of pelvic organ prolapse in women. Cochrane Database Syst Rev 2013;30;4:CD004014.

2. Gutman R, Maher C. Uterine-preserving POP surgery. Int Urogynecol J 2013;24:1803-13

3. Detollenaere RJ, den Boon J, Stekelenburg J et al. Sacrospinous hysteropexy versus vaginal hysterectomy with suspension of the uterosacral ligaments in treatment of uterine prolapse stage two or higher: multicentre randomised noninferiority trial. BMJ 2015;351:h3717

4. Detollenaere RJ, den Boon J, Kluivers KB, Vierhout $M E$, van Eijndhoven HW. Surgical management of pelvic organ prolapse and uterine descent in the Netherlands. Int Urogynecol J 2013;24:781-8

5. Wu MP, Long CY, Huang KH, Chu CC, Liang CC, Tang CH. Changing trends of surgical approaches for uterine prolapse: an 11-year population-based nationwide descriptive study. Int Urogynecol J 2012;23:865-72

6. Khan AA, Eilber KS, Clemens JQ, Wu N, Pashos CL, Anger JT. Trends in management of pelvic organ prolapse among female Medicare beneficiaries. Am J Obstet Gynecol 2015; 212(4): 463

7. van der Vaart $\mathrm{CH}$, de Leeuw JR, Roovers JP, Heintz AP. Measuring health-related quality of life in women with urogenital dysfunction: the urogenital distress inventory and incontinence impact questionnaire revisited. Neurourol Urodyn 2003:22:97-104

8. Roovers JP, van der Bom JG, van der Vaart $\mathrm{CH}$, Heintz AP. Prediction of findings at defecography in patients with genital prolapse. BJOG 2005:112:1547-53

9. Lamers LM, Stalmeier PF, McDonnell J, Krabbe PF. van Busschbach JJ. Measuring the quality of life in economic evaluations: the Dutch EQ-5D tariff. Ned Tijdschr Geneeskd 2005;149:1574-8.

10. Dietz V, Schraffordt Koops SE, van der Vaart $\mathrm{CH}$. Vaginal surgery for uterine descent; which options do we have? A review of the literature. Int Urogyn J; 2009:20:349-56

11. Altman D, Granath $F$, Cnattingius $S$, Falconer C: Hysterectomy and risk of stress-urinaryincontinence surgery: nationwide cohort study. Lancet 2007:370:1494-9.

12. Dietz $\mathrm{V}$, van der Vaart $\mathrm{CH}$, van der Graaf $\mathrm{Y}$, Heintz P, Schraffordt Koops SE. One-year follow-up after sacrospinous hysteropexy and vaginal hysterectomy for uterine descent: a randomized study. Int Urogyn J 2010;2:209-16.

13. De Boer TA, Milani AL, Kluivers KB, Withagen MI, Vierhout ME. The effectiveness of surgical correction of uterine prolapse: cervical amputation with uterosacral ligament plication (modified Manchester) versus vaginal hysterectomy with high uterosacral ligament plication. Int Urogyn J 2009;20:1313-19

14. Korbly NB, Kassis NC, Good MM et al. Patient preferences for uterine preservation and hysterectomy in women with pelvic organ prolapse. Am J Obstet Gynecol 2013;209:470. e1-6.

15. Frick AC, Barber MD, Paraiso MF, Ridgeway B, Jelovsek JE, Walters MD. Attitudes toward hysterectomy in women undergoing evaluation for uterovaginal prolapse. Female Pelvic Med Reconstr Surg 2013;19: 103-9.

16. Wong K, Jakus-Waldman S, Yazdany T. Patient beliefs regarding hysterectomy in women seeking surgery for pelvic organ prolapse: findings in a predominantly Hispanic population. Female Pelvic Med Reconstr Surg 2014;20:267-71.

17. Ellerkmann RM, Cundiff GW, Melick CF, Nihira MA Leffler K, Bent AE. Correlation of symptoms with location and severity of pelvic organ prolapse. Am J Obstet Gynecol. 2001;185:1332-7.

18. Jha S, Moran P. The UK national prolapse survey: 5 years on. Int Urogynecol J 2011;22:517-28.

19. Vanspauwen R, Seman E, Dwyer P. Survey of current management of prolapse in Australia and New Zealand. Aust N Z J Obstet Gynaecol 2010:50:262-7. 


\section{HYSTEROPEXY IN THE TREATMENT OF UTERINE}

PROLAPSE STAGE 2 OR HIGHER:

A MULTICENTER RANDOMISED CONTROLLED NON-INFERIORITY TRIAL COMPARING LAPAROSCOPIC SACROHYSTEROPEXY WITH VAGINAL SACROSPINOUS HYSTEROPEXY (LAVA-TRIAL, STUDY PROTOCOL)

M.N. van IJsselmuiden A.L. Coolen R.J. Detollenaere

J. den Boon

M.Y. Bongers

G. van de Pol

A. Vollebregt

C.M. Radder

J. Deprest

H.W.F. van Eijndhoven 


\section{ABSTRACT}

\section{BACKGROUND}

Pelvic organ prolapse is a common health problem: the lifetime risk of undergoing surgery for pelvic organ prolapse by the age of 85 years is $19 \%$. Pelvic organ prolapse has significant negative effects on a woman's quality of life. Worldwide, vaginal hysterectomy is the leading treatment method for patients with symptomatic uterovaginal prolapse. Several studies have shown that vaginal sacrospinous hysteropexy and laparoscopic sacrohysteropexy are safe and effective alternatives in treating uterine descent. To date, it is unclear which of these techniques leads to the best operative result and the highest patient satisfaction. Therefore, we conducted the LAVA trial.

\section{METHODS}

The LAVA trial is a randomized controlled multicenter non-inferiority trial. The study compares laparoscopic sacrohysteropexy with vaginal sacrospinous hysteropexy in women with uterine prolapse stage 2 or higher. The primary outcome of this study is surgical success of the apical compartment at 1 and 5 years follow-up. Secondary outcomes are subjective improvement on urogenital symptoms and quality of life (assessed by disease-specific and general quality of life questionnaires), complications following surgery, hospital stay, post-operative recovery, sexual functioning and costs-effectiveness. Evaluation will take place pre-operatively, and 6 weeks, 6 months, 12 months and annually till 60 months after surgery. Validated questionnaires will be used.

Analysis will be performed according to the intention to treat principle. Based on comparable recurrence rates of $3 \%$ and a non-inferiority margin of $10 \%, 62$ patients are needed in each arm to prove the hypothesis with a $95 \%$ confidence interval.

\section{DISCUSSION}

The LAVA trial is a randomized controlled multicenter non-inferiority trial that will provide evidence whether the efficacy of laparoscopic sacrohysteropexy is noninferior to vaginal sacrospinous hysteropexy in women with symptomatic uterine prolapse stage 2 or higher.

\section{TRIAL REGISTRATION}

Netherlands Trial Register (NTR): NTR4029

\section{BACKGROUND}

Pelvic organ prolapse (POP) is defined as the descent of one or more of the pelvic organs. $\mathrm{POP}$ is a common health problem. As life expectancy increases, a significantly greater number of women will present with POP requiring surgery. Given a life expectancy of 85 years, the lifetime risk of women undergoing a surgery for POP is 19\% [1]. POP has significant negative effects on a woman's quality of life, ranging from physical discomfort, psychological and sexual complaints to occupational and social limitations.

The etiology of POP is multifactorial: vaginal childbirth, advancing age and increasing body-mass index are the most consistent risk factors [2,3].

Women can present with prolapse of one or more compartments. Symptoms are commonly attributed to the anatomical compartments that are involved. Anterior vaginal wall prolapse concerns the urethra and/or the bladder (uretrocele, cystocele). Apical prolapse entails either the uterus or vaginal cuff after hysterectomy. Posterior vaginal wall prolapse involves the rectum (rectocele) but can also include the small intestines (enterocele). This study will focus on the treatment of uterine prolapse.

Worldwide, vaginal hysterectomy is the most frequently used treatment method for patients with symptomatic uterovaginal prolapse: it appeared to be the procedure of choice in the United Kingdom (82\%) [4] and in Australia and New Zealand (79\%) [5]. Although the literature is inconclusive, it has been suggested that hysterectomy may cause nerve supply damage and disrupt supportive structures of the pelvic floor, subsequently increasing the risk of future vaginal vault prolapse [6]. Furthermore, hysterectomy alone fails to address the underlying deficiencies in pelvic support causing uterine prolapse. Up to $11.6 \%$ of women undergoing hysterectomy subsequently present with vaginal vault prolapse [7] and the frequency of posthysterectomy vault prolapse requiring surgical repair is estimated between 6 and $8 \%$ [8].

Many other techniques for the treatment of uterine descent have been described, including vaginal, abdominal and laparoscopic procedures.

One procedure for uterine descent with uterine preservation is vaginal sacrospinous hysteropexy. In this procedure, the uterus is suspended to the sacrospinous ligaments with permanent sutures. In several studies, this technique was demonstrated to be a safe procedure for the primary treatment of uterine descent ${ }^{[9-11]}$. In a prospective observational study of 72 women with uterine descent, sacrospinous hysteropexy 
significantly reduced all urogenital and several defecatory symptoms and significantly improved quality of life. It also cured the uterine descent in $93.1 \%$ of women [12]. The procedure is associated with a few serious complications. The most common complication is buttock pain on the side where the sacrospinous sutures have been passed, this occurs in approximately $10-15 \%$ of the women, but resolves typically in days to months. One prospective study and one retrospective study comparing vaginal hysterectomy to vaginal sacrospinous hysteropexy demonstrated no significant difference in anatomical outcome, while hospital stay was shorter, less pain was experienced and recovery was quicker in the latter group [13-15]. These studies conclude that vaginal sacrospinous hysteropexy is associated with a faster complete recovery compared to vaginal hysterectomy. In both groups high recurrence rates of anterior vaginal wall prolapse were demonstrated (50\% in hysterectomy group vs. $65 \%$ in sacrospinous hysteropexy group) [15]. It is believed that the change in axis of the vagina after a sacrospinous hysteropexy predisposes to an increased risk of prolapse of the anterior compartment [16].

Another procedure for uterine descent with uterine preservation is laparoscopic sacrohysteropexy. In this procedure, the uterus is elevated by attaching the cervix to the sacral promontory using a mesh. One prospective study suggests that sacrohysteropexy is a safe and effective procedure. One out of 13 women $(7.7 \%)$ had a recurrence of uterine prolapse (mean follow-up 15.6 months) [17]. However, in this study the sacrohysteropexy was performed by laparotomy.

Another prospective study in which laparoscopic sacrohysteropexy was performed in 51 women showed no objective recurrence of uterine prolapse during a 10 weeks follow-up [18]. Laparoscopic sacrohysteropexy may have some complications. Typically, the use of a synthetic mesh implies a theoretical risk of erosion into an adjacent structure, or adhesion of bowel to the mesh, with subsequent development of symptoms and eventually signs of acute or chronic obstruction. Current experience anticipates a mesh related complication in less than $1 \%$ using a polypropylene mesh in abdominal correction of pelvic organ prolapse [19].

Based on literature, it seems that uterus suspension in case of uterine descent could be an effective procedure and avoid the limitations of hysterectomy. To our knowledge, a study comparing vaginal sacrospinous hysteropexy with laparoscopic sacrohysteropexy for the treatment of uterine descent has not been performed yet. It is unclear which of these treatment modalities results in the best anatomic correction and the highest patient satisfaction. Herein, we report on a multicenter randomized controlled trial to determine whether the efficacy of laparoscopic sacrohysteropexy is similar to vaginal sacrospinous hysteropexy in women with symptomatic uterine prolapse POP-Q stage 2 or higher.

\section{METHODS/DESIGN}

\section{STUDY OBJECTIVES}

The objective of this study is to determine whether laparoscopic sacrohysteropexy in women with uterine descent stage 2 or higher improves outcome in terms of recurrence of prolapse, quality of life, complications, hospital stay, post-operative recovery, sexual functioning and costs-effectiveness compared to vaginal sacrospinous hysteropexy.

\section{HYPOTHESIS}

Based on the literature, we hypothesize that there is no difference in surgical success rate of the apical compartment between laparoscopic sacrohysteropexy and vaginal sacrospinous hysteropexy in symptomatic women with uterine descent POP-Q stage 2 or higher. Possibly, laparoscopic sacrohysteropexy might be associated with quicker post-operative recovery, less dyspareunia post-operatively and lower recurrence rates with respect to the anterior vaginal wall.

\section{STUDY DESIGN}

The LAVA-trial is a prospective, randomized controlled non-blinded clinical trial conducted with the aim to determine non-inferiority of the primary endpoint (surgical success of the apical compartment) between laparoscopic sacrohysteropexy and vaginal sacrospinous hysteropexy. The study will be an open label study, as it is impossible to blind the participating patients and the health care workers for the surgical procedure to which the woman is allocated. However, follow-up at six months, one and five years will be done by a physician not involved in the surgery. Post-operative followup will take place after 6 weeks, 6 months, 12 months and annually thereafter until 5 years. Patients will undergo a standard gynecological examination (including a POP-Q examination) and fill in questionnaires. The study design is presented in figure 1.

\section{PRIMARY AND SECONDARY OUTCOMES}

Surgical success of the middle compartment at 1 and 5 years follow-up in both study groups will be considered as the primary outcome. Success is defined as position of the cervix at or above the mid-vagina $(C<-T V L / 2)$, no symptoms (defined as no symptoms of vaginal bulging and protrusion on the validated questionnaire) and no 
reoperation or pessary use for recurrent apical prolapse [20]. Failure in one of these areas will constitute a failure. Secondary outcomes of this study include prolapse of the anterior and posterior compartment, subjective outcome and improvement in general and disease-specific quality of life, complications, hospital stay, postoperative recovery, sexual functioning, cost-effectiveness and uterine issues (e.g. abnormal bleeding, cervical dysplasia) and the need for subsequent hysterectomy.

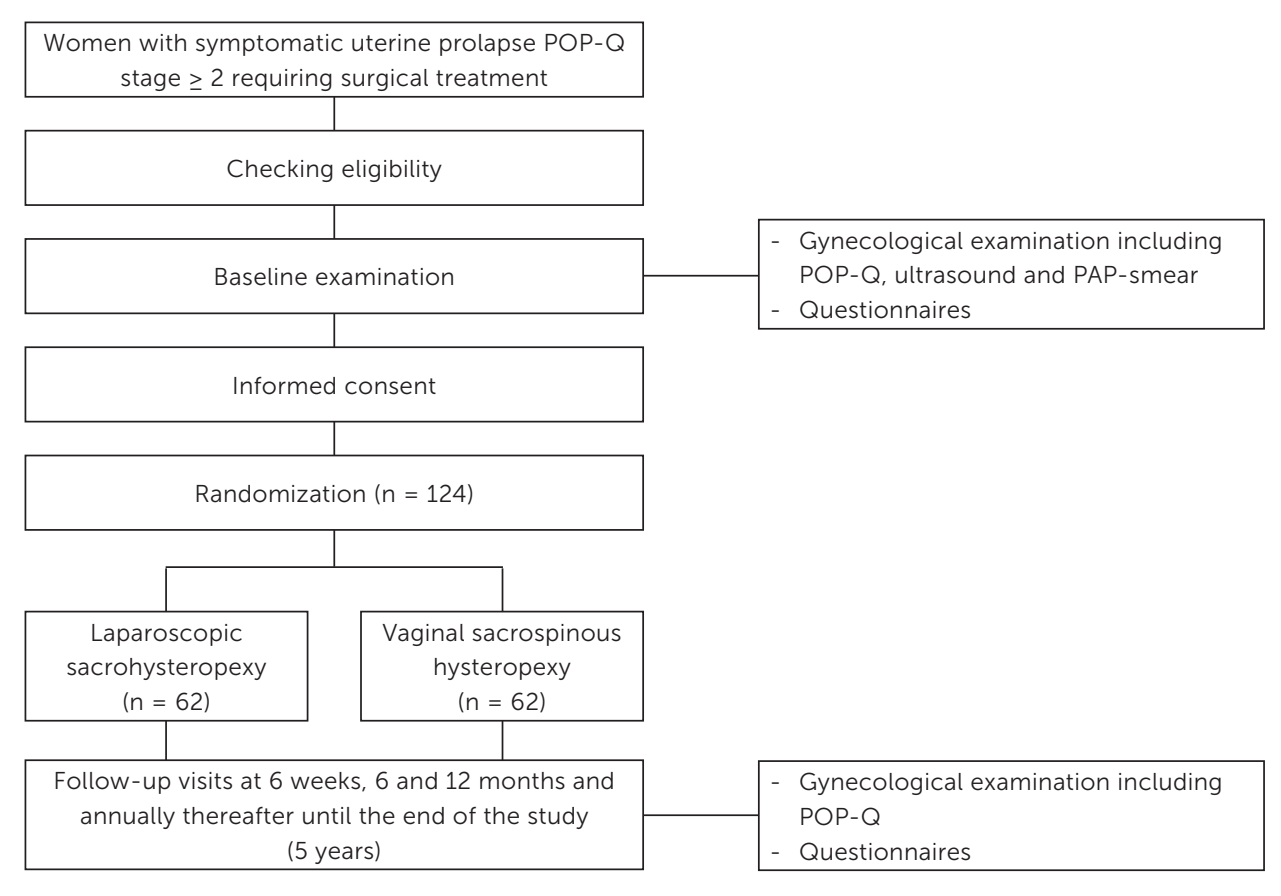

FIGURE 1. Study design

\section{STUDY POPULATION AND RECRUITMENT}

All women seeking treatment for symptomatic pelvic organ prolapse with uterine descent POP-Q stage 2 or higher are eligible for inclusion in the LAVA trial. A total of 124 women will participate in this trial. Patients with co-existing anterior/posterior defects or concomitant incontinence surgery can be included.

Women with previous pelvic floor or prolapse surgery, known malignancy of the cervix or cervical dysplasia, language barriers, a wish to preserve fertility, presence of immunological or hematological disorders interfering with recovery after surgery, contraindications for laparoscopic surgery (e.g. ileus, risk of severe adhesions), abnormal ultrasound findings of uterus or ovaries which cause symptoms and/ or require treatment, abnormal uterine bleeding that requires surgical treatment, postmenopausal bleeding in the past year and women who are unwilling to return for follow-up are excluded from this study. Inclusion of women with cervical elongation will be left to the discretion of the enrolling surgeon. In case the surgeon believes that surgical shortening of the cervix or even vaginal hysterectomy is more appropriate because of a distinct elongation the patient should not be enrolled. Assessment for eligibility is performed by a gynecologist of the participating hospital. Patients eligible for this study are counseled about the long duration of follow-up required for this study. Subsequently, written patient information is provided, which contains information on the objectives, design, methods, possible advantages and disadvantages of the study treatments, and information that non-co-operation with the study or withdrawal will not have consequences for their treatment. An interval of at least one week between the primary visit and the next appointment allows sufficient time for women to think about participation. Before randomization, written informed consent is obtained.

\section{PARTICIPATING HOSPITALS}

One university hospital in Belgium and five Dutch general hospitals will participate in this trial and enroll patients.

\section{RANDOMIZATION}

After patients have consented for participating in this study, randomization will be performed by the coordinating researcher centrally through a website using a computer-generated randomization table. The subjects are assigned in a 1:1 ratio to either vaginal sacrospinous hysteropexy or laparoscopic sacrohysteropexy. The randomization will be stratified according to the center and severity of prolapse (POP-Q stage 2, 3 or 4). The order of outcomes is unknown to the investigators or to the participating gynecologists. Data will be kept anonymous. The subjects will be informed about the allocated operative procedure shortly after the randomization. Subjects that withdraw after randomization will be treated according to the preference of the gynecologist.

\section{DATA COLLECTION}

All patients will undergo routine gynecological examination, which is part of the standard procedure before surgery. This examination includes pelvic ultrasound to exclude uterine or ovarian disease, routine PAP-smear and vaginal inspection in $45^{\circ}$ semi-upright position for staging uterovaginal prolapse by a POP-Q examination. Maximum prolapse is demonstrated and identified by asking the patient to cough and 
has been developed for both surgical procedures. All procedures are performed according to this standardized protocol and also all participating hospitals use the same materials for the procedures (e.g. sutures, mesh). At least twenty procedures must have been performed by participating gynecologists to eliminate a learning curve effect. It is allowed for participating gynecologists to partner with a colleague gynecologist: one gynecologist can perform the laparoscopic sacrohysteropexy and the other gynecologist can perform the vaginal sacrospinous hysteropexy. of life questionnaires (RAND 36, Euroqol 5D, Urogenital Distress Inventory, Defecatory
Distress Inventory, Incontinence Impact questionnaire) and two questionnaires regarding sexual functioning (Pelvic Organ Prolapse/Urinary Incontinence Sexual Questionnaire and selected items from the "Vragenlijst Seksuele Disfuncties") [2129]. Preoperative urodynamic evaluation is only performed in women with bladder dysfunction.

During hospitalization and in the first 6 weeks after surgery patients are asked to keep a diary which contains the following items: postoperative pain measured by Visual Analogue Score (VAS), used pain medication and the RI-10 recovery questionnaire. The RI-10 recovery questionnaire is a validated quality of life questionnaire measuring subjective postoperative recovery [30]. To prevent a recall bias as much as possible, patients will be phoned two and four weeks after surgery as a reminder to fill in the diary. After surgery, patients will visit the hospital at 6 weeks (routine post-operative consultation), 6 months, 12 months and yearly thereafter. The total duration of the follow-up is 5 years. Each check-up includes a standardized written questionnaire regarding quality of life and sexual functioning (similar to the questionnaires at baseline) and a clinical examination (including POP-Q)

The validated questionnaires will be administered by the participating hospitals (baseline and 6 weeks postoperative) and by the coordinating hospital (6 months postoperative and yearly thereafter).

\section{INTERVENTIONS}

Eligible women will be randomly allocated to receive either a laparoscopic sacrohysteropexy or a vaginal sacrospinous hysteropexy. The vaginal procedure can be performed under general or spinal anesthesia, according to the preference of patient and anesthesiologist. The laparoscopic procedure will be performed under general anesthesia. All women receive peri-operative antibiotics and thrombosis prophylaxis. Post-operatively a bladder catheter is placed and removed after one day. Patients will receive analgesics if necessary in accordance with local hospital protocol. All patients are advised to abstain from heavy physical work for a minimal period of 6 weeks.

In order to standardize both procedures and prevent variation in procedures, a protocol

\section{LAPAROSCOPIC SACROHYSTEROPEXY}

The patient is placed in lithotomy position. An uterine manipulator (Clearview, Clinical Innovations LLC, Murray, UT, USA) is placed to provide visualization of the surgical site. Four laparoscopic ports (umbilical, suprapubic, two lateral ports) will be placed and a pneumoperitoneum will be created. The colon sigmoid can be removed from the operating field by attaching it to the abdominal wall by a suture through some plica epiploica. The peritoneum over the sacral promontory will be incised; the right ureter will be identified. Each broad ligament at the level of the cervico-uterine junction will be opened. The vesico-uterine peritoneum will be incised and the bladder will be dissected distally for $2-3 \mathrm{~cm}$. A bifurcated polypropylene flat mesh (Gynemesh, Ethicon bilaterally through windows created in the broad ligaments. Permanent sutures (Mersilene 2.0, Ethicon, Sommerville, NJ, USA) will be placed through the arms of the mesh and the anterior cervix (2-3 sutures) and the posterior cervix (4 sutures). The surgeon is allowed to place the mesh further down the anterior and posterior vaginal wall to treat compartment specific prolapses. The mesh will be tacked to the sacral promontory using $5.3 \times 3.7 \mathrm{~mm}$ titanium staples (Endoscopic Multifeed Stapler-20, Ethicon Inc, Sommerville, NJ, USA) to elevate the uterus (3 staples). The peritoneum will be closed with $5.3 \times 3.7 \mathrm{~mm}$ titanium staples (Endoscopic Multifeed Stapler-20, Ethicon, Sommerville, NJ, USA) covering the promontory part of the mesh and a running suture (Vicryl 2.0, Ethicon Inc, Sommerville, NJ, USA) covering the cervical part of the mesh. The laparoscopic ports will be removed and the wounds will be closed. Vaginal examination after the laparoscopic hysteropexy is part of the protocol and additional anterior and/or posterior colporrhaphy or incontinence surgery can be performed if necessary, according to the standard procedures of the hospital.

\section{VAGINAL SACROSPINOUS HYSTEROPEXY}

The patient is placed in lithotomy position. Access to the sacrospinous ligament is obtained through the pararectal space. The posterior vaginal wall will be incised and separated from the rectum. The right ischial spine will be localized digitally and after Inc, Sommerville, NJ, USA) will be used. The arms of the mesh will be introduced 
retractor positioning, the ligament is made visible through blunt dissection. Two permanent sutures (Prolene 1.0, Ethicon Inc, Sommerville, NJ, USA) will be placed under direct vision through the right sacrospinous ligament at least $2 \mathrm{~cm}$ from the ischial spine. Hereafter, an additional anterior and/or posterior colporrhaphy or incontinence surgery can be performed, according to the standard procedures of the hospital. The permanent sutures will be placed through the posterior side of the cervix and two thirds of the posterior vaginal wall will be closed with absorbable sutures (Vicryl 2.0, Ethicon Inc, Sommerville, NJ, USA). The permanent sutures will be tightened and the cervix will be redressed. The remainder of the vaginal wall will be closed.

\section{STATISTICAL ANALYSIS}

Sample size and power calculations

Sample size calculation was performed using Sample Power 2.0 (SPSS inc. Chicago, IL, USA). The aim of the study is to clarify whether laparoscopic sacrohysteropexy and vaginal sacrospinous hysteropexy do not differ significantly in surgical success rates of the apical compartment. We aim to demonstrate non-inferiority of the laparoscopic sacrohysteropexy at both 1 and 5 year (i.e. two tests on the primary endpoint will be performed). Two groups of 55 patients will be included to yield a $80 \%$ power for a non-inferiority margin of $10 \%$ assuming a recurrence rate of prolapse at the apical compartment of $3 \%$. Taking into account $10 \%$ attrition, a number of 62 patients will be included in each study arm. This dropout can be expected when patients, after randomization, are unhappy with their allocated treatment and choose to end their participation in the study or due to incomplete or lost data. A total number of 124 women will be included in this study.

\section{Data analysis}

The analysis will be performed by intention to treat, and stratified for center and severity of prolapse. Patient characteristics will be summarized using descriptive statistics for continuous variables (mean \pm standard deviation, minimum, maximum and sample size) and frequency tables for categorical variables (numbers and percentages). Statistical analysis of the data will be conducted at 12 and 60 months follow-up.

Surgical success of the apical compartment at 1 and 5 years follow-up in both study groups will be considered as the primary outcome. Success is defined as position of the cervix at or above the mid-vagina $(C<-T V L / 2)$, no symptoms (defined as no symptoms of vaginal bulging and protrusion on the validated questionnaire) and no reoperation or pessary use for recurrent apical prolapse. Failure in one of these areas will constitute a failure. Non-inferiority of laparoscopic sacrohysteropexy to vaginal sacrospinous hysteropexy will be concluded if the $95 \%$ confidence interval (Cl) does not exceed the non-inferiority margin of $10 \%$ (figure 2, scenario B). If the whole $95 \% \mathrm{Cl}$ exceeds the non-inferiority margin of $10 \%$, the laparoscopic sacrohysteropexy will be considered to be inferior (scenario C). If the $95 \% \mathrm{Cl}$ for the difference in surgical success rates lies left from zero, it can be concluded that there is evidence of superiority of laparoscopic sacrohysteropexy over vaginal sacrospinous hysteropexy (scenario A) [31,32].

\section{Ethics}

The study is conducted in accordance with the principles of the Declaration of Helsinki. The LAVA trial has been approved by the Medical Ethical Committee of the Isala Klinieken Zwolle (METC 13/0320) and the local Ethical Committees of the participating centers. Prior to randomization, informed consent will be obtained.

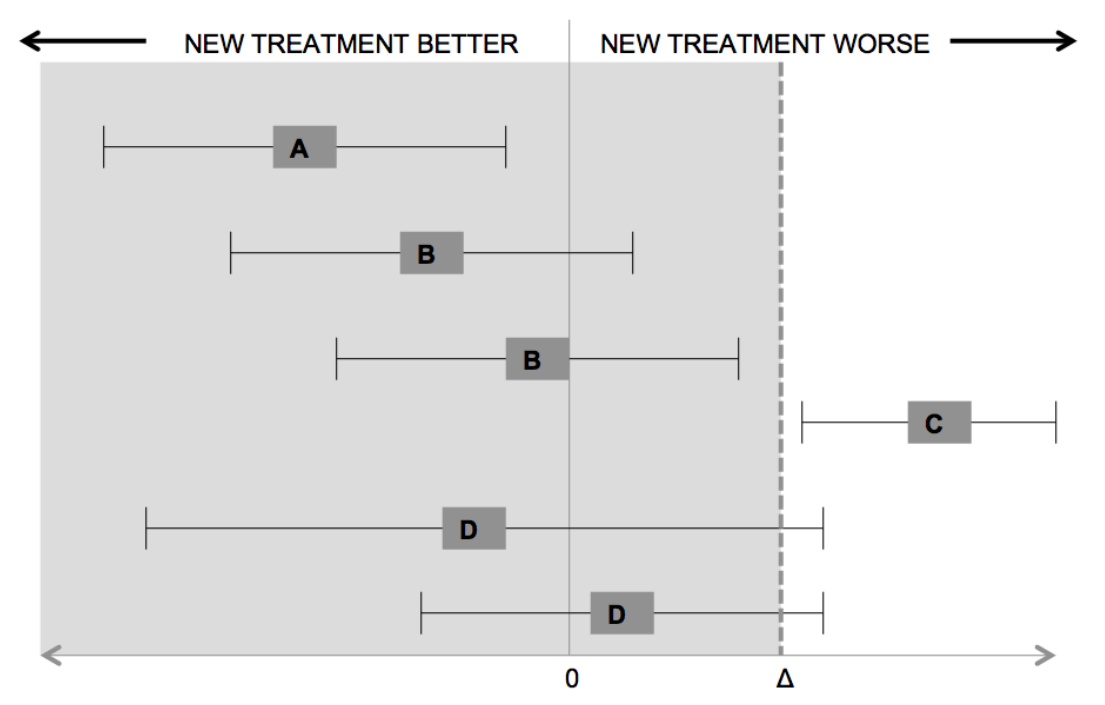

FIGURE 2. Interpreting results of non-inferiority trials. Interpreting results of non-inferiority trials: $\Delta$ stands for the non-inferiority margin. The results of a non-inferiority trial can conclude superiority (A) as the $95 \%$ confidence interval (CI) lies left from zero (A), non-inferiority as the $95 \% \mathrm{Cl}$ does not exceed $\Delta(B)$ and inferiority as the $95 \%$ exceed $\Delta$ (C). If the $95 \% \mathrm{Cl}$ included $\Delta$, the results are inconclusive (D). 


\section{DISCUSSION}

In several studies it has been shown that uterine suspension in case of uterine descent is a safe and effective procedure compared to hysterectomy ${ }^{[1,13-15,18]}$. To our knowledge, a study that compares vaginal sacrospinous hysteropexy with laparoscopic sacrohysteropexy for the treatment of uterine descent has not been performed yet. It is unclear which treatment option gives the best anatomical outcome and the highest patient satisfaction. Therefore, a sufficiently powered randomized controlled trial with long-term follow-up is required to provide evidence based decisions on the preferred treatment. If non-inferiority in surgical success is found, the comparison of the secondary outcomes will be essential in selecting the preferred strategy.

\section{REFERENCES}

1. Smith FJ, Holman CD, Moorin RE, Tsokos N: Lifetime risk of undergoing surgery for pelvic organ prolapse. Obstet Gynecol 2010,116(5):1096-100.

2. Jelovsek JE, Maher C, Barber MD: Pelvic organ prolapse. Lancet 2007, 369:1027-38.

3. Dietz HP: The aetiology of prolapse. Int Urogynecol J 2008, 19:1323-1329.

4. Jha S, Moran P: The UK national prolapse survey: 5 years on. Int Urogynecol J 2011, 22(5): 517-528.

5. Vanspauwen R, Seman E, Dwyer P: Survey of current management of prolapse in Australia and New Zealand. Aust N Z J Obstet Gynaecol 2010. 50(3): 262-7.

6. Diwan A, Rardin CR, Strohsnitter WC, Weld A. Rosenblatt P, Kohli N: Laparoscopic uterosacral ligament uterine suspension compared with vaginal hysterectomy with vaginal vault suspension for uterovaginal prolapse. Int Urogynecol J 2005, 17: 79-83.

7. Marchionni M, Bracco GL, Checcucci V Carabaneanu A, Coccia EM, Mecacci F, Scarselli G: True incidence of vaginal vault prolapse. Thirteen years of experience. J Reprod Med 1999 44(8):679-684.

8. Aigmueller T, Dungl A, Hinterholzer S, Geiss I, Riss P: An estimation of the frequency of surgery for posthysterectomy vault prolapse. Int Urogynecol J 2010, 21(3): 299-302.

9. Dietz V, de Jong J, Huisman M, Schraffordt Koops S, Heintz P, van der Vaart H: The effectiveness of the sacrospinous hysteropexy for the primary treatment of uterovaginal prolapse. Int Urogynecol J 2007, 18:1271-76.

10. Hefni M, El-Toukhy T, Bhaumik J, Katsimanis E: Sacrospinous cervicocolpopexy with uterine conservation for uterine prolapse in eldery women: an evolving concept. Am J Obstet Gynecol 2003, 188(3): 645-50.

11. Detollenaere RJ, den Boon J, Stekelenburg $J$, Kluivers KB, Vierhout ME, van Eijndhoven HWF: Short term anatomical results of a randomized controlled non inferiority trial comparing sacrospinous hysteropexy and vaginal hysterectomy in treatment of uterine prolapse stage 2 or higher. Int Urogynecol J 2013, 24(Suppl): S1-S2

12. Dietz V, Huisman M, de Jong JM, Heintz PM, van der Vaart $\mathrm{CH}$ : Functional outcome after sacrospinous hysteropexy for uterine descensus. Int J Urogynecol 2008, 19:747-52.

13. Brummen $\mathrm{HJ}$, van de Pol G, Aalders CIM, Heintz APM, van der Vaart CH: Sacrospinous hysteropexy compared to vaginal hysterectomy as primary surgical treatment for a descensus uteri: effect on urinary symptoms. Int Urogynecol J 2003, 14:350-55.

14. Maher CF, Cary MP, Slack MC, Murray CJ, Milligan $M$, Schluter $P$ : Uterine preservation or hysterectomy at sacrospinous colpopexy for uterine prolapse? Int Urogynecol J 2001, 12: 38185.

15. Dietz $\mathrm{V}$, van der Vaart $\mathrm{CH}$, van der Graaf $\mathrm{Y}$, Heintz AP, Schraffordt Koops SE: One-year followup after sacrospinous hysteropexy and vaginal hysterectomy for uterine descent: a randomized study. Int Urogynecol J 2010, 21(2): 209-16.

16. Aigmueller Th, Riss P, Dungl A, Bauer H. Longterm follow-up after vaginal sacrospinous fixation: patient satisfaction, anatomical results and quality of life. Int Urogynecol J 2008, 19:965969.

17. Leron E, Stanton SL: Sacrohysteropexy with 
synthetic mesh for the management of uterovaginal prolapse. BJOG 2001, 108: 629-33.

18. Price N, Slack A, Jackson SR: Laparoscopic hysteropexy: the initial results of a uterine suspension procedure for uterovaginal prolapse. BJOG 2010, 117: 62-68.

19. Murphy M: Use of mesh and materials in pelvic floor surgery. Obstet Gynecol Clin N Am 2009, 36:615-35. Ware JE, Kosinski M, Keller SD: SF36 physical and mental component summary measures - a users' manual. Boston: New England Medical Center, The Health Institute; 1994.

20. Barber MD, Brubaker L, Nygaard I, Wheeler TL 2nd, Schaffer J, Chen Z, Spino C. Defining success after surgery for pelvic organ prolapse. Obstet Gynecol 2009, 114:600-9

21. Van der Zee KI, Sanderman R: Het meten van de gezondheidstoestand met de Rand-36, een handleiding. Groningen: Noorderlijk centrum voor gezondheidsvraagstukken; 1993.

22. Van der Vaart $\mathrm{CH}$, de Leeuw JR, Roovers JP, Heintz AP: Measuring health-related quality of life in women with urogenital dysfunction: the urogenital distress inventory and incontinence impact questionnaire revisited. Neurourol Urodyn 2003, 22: 97-104.

23. Roovers JP, van der Bom JG, van der Vaart CH, Heintz AP: Prediction of findings at defecography in patients with genital prolapse. BJOG 2005 , 112:1547-53.

24. Dolan P: Modeling valuations for EuroQol health states. Med Care 1997, 35: 1095-1108.

25. Lamers LM, Stalmeier PF, McDonnell J, Krabbe PF, van Busschbach JJ: Measuring the quality of life in economic evaluations: the Dutch EQ-5D tariff. Ned Tijdschr Geneeskd 2005, 149: 1574-78.

26. Espuña Pons M: Sexual health in women with pelvic floor disorders: measuring the sexual activity and function with questionnaires - a summary. Int Urogynecol J Pelvic Floor Dysfunct 2009, 20:S65-S71.

27. Schweitzer KJ, de Jong M, Milani AL: Prolaps en seks: hoe meten we de relatie? NTOG 2008, 121:79-82.

28. Vroege JA: De vragenlijst voor het signaleren van seksuele dysfuncties (VSD). Bruikbaarheid in de klinische praktijk. Delft; 2003.

29. Ware JE, Kosinski M, Keller SD: SF-36 physical and mental component summary measures - a users' manual. In Boston. The Health Institute: New England Medical Center; 1994.

30. Kluivers KB, Hendriks JC, Mol BM, Bongers MY, Vierhout ME, Brölmann HA, de Vet HC: Clinimetric properties of 3 instruments measuring postoperative recovery in a gynecologic surgical population. Surgery 2008, 144(1):12-21.

31. Christensen E: Methodology of superiority vs. equivalence trials and non-inferiority trials. J Hepatol 2007, 46(5):947-54.

32. Soonawala D, Dekkers OM: Non-inferiority trials: tips for the critical reader. Research methodology 3. Ned Tijdschr Geneeskd 2012, 156(19):A4665. 


\section{HYSTEROPEXY IN THE TREATMENT OF UTERINE \\ PROLAPSE STAGE 2 OR HIGHER:}

LAPAROSCOPIC SACROHYSTEROPEXY VERSUS SACROSPINOUS HYSTEROPEX - A MULTICENTRE RANDOMISED TRIAL (LAVA-TRIAL)

M.N. van IJsselmuiden A.M.J van Oudheusden

G. van de Pol

A. Vollebregt

C.M. Radder

S. Housmans

S.M.J. van Kuijk

J. Deprest

M.Y. Bongers

H.W.F. van Eijndhoven 


\section{ABSTRACT}

\section{OBJECTIVE}

To investigate whether laparoscopic sacrohysteropexy (LSH) is non-inferior to vaginal sacrospinous hysteropexy (SSHP) in the surgical treatment of uterine prolapse.

\section{DESIGN}

Multicentre randomised controlled non-blinded non-inferiority trial.

\section{SETTING}

5 non-university teaching hospitals in the Netherlands, one university hospital in Belgium.

\section{POPULATION}

126 women with uterine prolapse stage 2 or higher undergoing surgery without previous pelvic floor surgery.

\section{METHODS}

Randomisation in a 1:1 ratio to LSH or SSHP, stratified per centre and severity of the uterine prolapse. The predefined inferiority margin was an increase in surgical failure rate of $10 \%$

\section{MAIN OUTCOME MEASURES}

Primary outcome was surgical failure, defined as reoccurrence of uterine prolapse ( $P O P-Q \geq 2$ ) with bothersome bulging/protrusion symptoms and/or repeat surgery or pessary at 12 months postoperative. Secondary outcomes were anatomical recurrence (any compartment), functional outcome and quality of life.

\section{RESULTS}

LSH was non-inferior for surgical failure $(n=1,1.6 \%)$ compared with SSHP $(n=2,3.3 \%$, difference $-1.7 \%, 95 \% \mathrm{Cl}-7.1$ to 3.7$) 12$ months postoperatively. Overall anatomical recurrences and quality of life did not differ. More bothersome symptoms of overactive bladder $(\mathrm{OAB})$ and faecal incontinence were reported after LSH. Dyspareunia was more frequently reported after SSHP.

\section{CONCLUSION}

LSH was non-inferior to SSHP for surgical failure of the apical compartment at 12 months' follow-up. Following LSH, bothersome $\mathrm{OAB}$ and faecal incontinence were more frequent, yet dyspareunia was less frequent.

\section{FUNDING}

Research grant from Isala research foundation, The Netherlands

\section{TRIAL REGISTRATION}

trialregister.nI NTR4029

\section{INTRODUCTION}

Pelvic organ prolapse (POP) is a common health problem and associated with a significant impairment in quality of life [1]. The lifetime risk of undergoing surgery for POP or urinary incontinence is $20 \%$ by the age of 80 years [2]. As result of an aging population, a significant increase in both the number of women with POP and those seeking care for POP is expected to occur over the next 20-40 years [3]. Consequently, surgical rates for POP and urinary incontinence in the United States are estimated to increase with $42.7 \%$ by 2050 [3].

Traditionally, uterine prolapse is treated by vaginal hysterectomy and suspension of the vaginal vault, despite the fact that the uterus is not the cause but only a passive structure in the development of prolapse $[4,5]$. However, uterus preservation techniques are gaining interest and more of these operations are being done in recent years [6-9]. In concert with this, more women express a preference for uterine preservation [10-12]. Women may want to avoid hysterectomy because they feel the uterus is important for a sense of self-esteem and plays a role in sexual satisfaction, the added surgical risk of hysterectomy, and/or a desire to preserve fertility $[10,12,13]$. In addition, data showing an added value of hysterectomy over uterus preservation are lacking.

Several studies have demonstrated that uterine suspension is as effective and safe as prolapse surgery including vaginal hysterectomy [13-16]. Laparoscopic, robotic, abdominal and vaginal procedures have been described to suspend the uterus. To date, no randomised controlled trials (RCTs) are available comparing these different routes [13]. Surgical access route and method of uterus suspension mostly depends on the preference of the surgeon. Whether or not these operations have comparable anatomical and functional outcomes remains unclear. 
Vaginal sacrospinous hysteropexy (SSHP) is the most studied technique for uterus suspension, its efficacy has been demonstrated in several RCTs [15-17]. However, laparoscopic sacrohysteropexy (LSH) has not been compared directly in a randomised trial against SSHP, although it seems to become increasingly popular [7,18]. Therefore, we tested the hypothesis that LSH is non-inferior to SSHP regarding surgical failure at 12 months' follow-up.

\section{METHODS}

\section{STUDY DESIGN}

The trial protocol of this study was previously published [19]. The study was approved by the ethics committee of the Isala Hospital (file number NL43801.075.13) and all additional centres, and was registered in the Dutch Trial Register (NTR4029). Briefly, all women with uterine prolapse at stage 2 or higher (uterine prolapse $1 \mathrm{~cm}$ above the hymen and beyond) and opting for surgical treatment were invited to participate. Women with coexisting anterior and/or posterior vaginal wall prolapse or concomitant incontinence surgery were also eligible. Exclusion criteria were previous pelvic floor or prolapse surgery, known cervical dysplasia/malignancy, language barriers, the wish to preserve fertility, presence of immunological or haematological disorders potentially interfering with postoperative recovery, contraindications for laparoscopic surgery (e.g. ileus, risk of severe adhesions), suspect findings of uterus and/or ovaries on ultrasound causing symptoms and/or requiring surgical treatment, abnormal uterine bleeding requiring surgical treatment, postmenopausal bleeding in the past year and unwillingness to return for follow-up. Participants were randomly assigned to LSH or SSHP.

Before enrolment, gynaecological examination was performed including pelvic ultrasound to exclude uterine or ovarian disease, a cervical smear test and vaginal inspection in $45^{\circ}$ semi-upright position for staging uterovaginal prolapse using the pelvic organ prolapse quantification system (POP-Q) [20].

Five non-university teaching hospitals in the Netherlands and one university hospital in Belgium participated in this study. In order to standardize surgery, a detailed protocol was developed for both operations during a specific study masterclass in which all centres participated. All procedures were performed according to this standardized protocol using the same materials (e.g. sutures, mesh). To eliminate a learning curve effect, each surgeon should have performed at least 20 procedures of each prior to the recruitment of the first patient for this study.
Written information was provided to eligible women and informed consent was obtained. Participants were then randomly assigned in a 1:1 ratio to either SSHP or LSH using a web-based computer application with generated computer randomisation tables in blocks of four. Randomisation was stratified per centre and severity of the uterine prolapse (POP-Q stage 2, 3 or 4). Surgeons and women were not blinded to the allocated surgical procedure. A physician trained in urogynaecology who was not involved in the management of patients performed the follow-up at 12 months.

\section{PRIMARY AND SECONDARY OUTCOMES}

The primary outcome was a composite outcome of surgical failure of the apical compartment at 12 months' follow-up, defined as reoccurrence of uterine prolapse (POP-Q $\geq$ stage 2) with bothersome bulging/protrusion symptoms and/or requiring therapy, whether it was repeat surgery or pessary [21]. Additional outcomes were anatomical failure in any compartment ( $P O P-Q \geq$ stage 2 in any compartment); surgical success defined as no prolapse beyond the hymen, neither bothersome bulge symptoms, and no therapy for recurrent prolapse within 12 months; and overall surgical failure, which was defined as $P O P-Q \geq$ stage 2 , pessary use, or repeat surgery for recurrent prolapse in any compartment within 12 months. Other secondary outcomes were functional outcome, quality of life and sexual functioning

\section{DATA COLLECTION}

POP measurements using the POP-Q system were performed at baseline, at six weeks, six months and 12 months after surgery. At those visits, women completed questionnaires regarding health-related and disease-specific quality of life (short form-365, Euroqol 5D, urogenital distress inventory, defecatory distress inventory and incontinence impact questionnaire) [22-25]. We defined the presence of bothersome bulge symptoms after surgery as any positive answer to any of the following two questions from the urogenital distress inventory: "Do you experience a sensation of bulging or protrusion from the vagina" and "Do you have a bulge or something protruding that you can see in the vagina?" in combination with a response "somewhat bothered" to "very much bothered" to the question "how much does this bother you?". To assess sexual functioning, the 12 -item pelvic organ prolapse/urinary incontinence sexual questionnaire was used [26]. Data were entered and registered using a webbased application facilitated by the Isala.

\section{INTERVENTIONS}

Women received perioperative antibiotics, thrombosis prophylaxis, a bladder catheter and postoperative analgesia according to local hospital protocols. LSH was performed 
under general anaesthesia. For SSHP, patients received general or spinal anaesthesia, according to patient or physician's preference. Participants were advised to abstain from heavy physical duties for six weeks.

Vaginal SSHP - The posterior vaginal wall was incised and separated from the rectum. The right ischial spine was localized digitally. After retractor positioning, the sacrospinous ligament was exposed through blunt dissection. Under direct vision, two permanent sutures (Prolene 1/0, Ethicon Inc., Sommerville, NJ, USA) were placed through the right sacrospinous ligament at least $2 \mathrm{~cm}$ from the ischial spine. After placing the polypropylene sutures through the posterior aspect of the cervix, these were tightened to redress the uterus. Finally, the posterior vaginal wall was closed using absorbable sutures. Concomitant anterior or posterior colporrhaphy and antiincontinence surgery were performed if indicated.

LSH - First a uterine manipulator (Clearview, Clinical Innovations LLC, Murray, UT, USA) was placed. After placing four laparoscopic ports (umbilical, suprapubic, two lateral ports) and creating a pneumoperitoneum, the peritoneum over the sacral promontory was incised. The broad ligament at the level of the cervico-uterine junction was opened. The vesico-uterine peritoneum was incised and the bladder dissected distally for 2-3 cm. The arms of a bifurcated polypropylene flat mesh (Gynemesh, Ethicon Inc., Sommerville, NJ, USA) were introduced bilaterally through windows created in the broad ligaments. Non-absorbable sutures were placed through the arms of the mesh and the anterior (2-3 sutures) and posterior (4 sutures) aspect of the cervix, respectively. The mesh was attached to the sacral promontory using three 5.3×3.7 mm titanium staples (Endoscopic Multifeed Stapler-20, Ethicon Inc., Sommerville, NJ, USA). The peritoneum was then closed using a resorbable running suture (Vicryl 2.0, Ethicon Inc., Sommerville, NJ, USA). After removing the laparoscopic ports, the wounds were closed. If indicated, anterior and/or posterior vaginal wall repair was performed laparoscopically (by extended dissection) or vaginally afterwards (anterior and/or posterior colporrhaphy). Furthermore, anti-incontinence surgery was performed if necessary.

\section{STATISTICAL ANALYSIS}

Sample size calculation was based on the primary outcome. We assumed a failure rate of 3\% based on outcomes of SSHP in a previous prospective study [17]. The noninferiority margin was set at $10 \%$. This means that when the upper limit of the $95 \%$ confidence interval $(\mathrm{Cl})$ for the estimated difference in recurrence rate after $\mathrm{LSH}$ versus SSHP exceeded $10 \%$, LSH would be regarded as inferior to SSHP. Assuming an absolute recurrence risk of $3 \%$ in both groups and a two-sided a risk of 0.05 , with two groups of 55 women each, the trial had $80 \%$ power with a prespecified non-inferiority margin of $10 \%$. Considering a 10\% loss to follow-up, we required 124 women, hence 62 in each study arm.

Data were analysed primarily according an intention to treat (ITT) principle. However, a per protocol (PP) analysis was done as well. In case of missing data on anatomical outcome at 12 months, this was reported, and we applied the last observation carried forward (LOCF) strategy with data at the six-month follow-up if available. If these data were not available, data of 6 weeks follow-up was used. In case the six-week followup was missing, we left the women out of the ITT-LOCF analysis. In case of missing questionnaires, we obtained information on the presence or absence of bothersome bulge symptoms from the 12-month follow-up visit. As sensitivity analysis we applied conservative imputation (worst-case scenario, failure) in which all patient lost to follow-up at the 12 months' visit were regarded as having experienced a recurrence.

The PP-analysis was performed on the primary and secondary outcomes for anatomical and surgical failure. This analysis included women who completed the entire treatment protocol as originally planned, with availability of the POP-Q scores at 12 months and absence of major deviations from the protocol.

For exploratory purposes of the results, chi-square or Fisher's exact test was used to compare proportions and a Mann-Whitney $U$ test was used to compare continuous variables between the groups. A p-value of less than 0.05 was considered to be significant. In case of statistical significance, linear regression was used to assess confounding with baseline values.

All statistical analyses were performed using SPSS for Windows (version 22.0.0.1). 


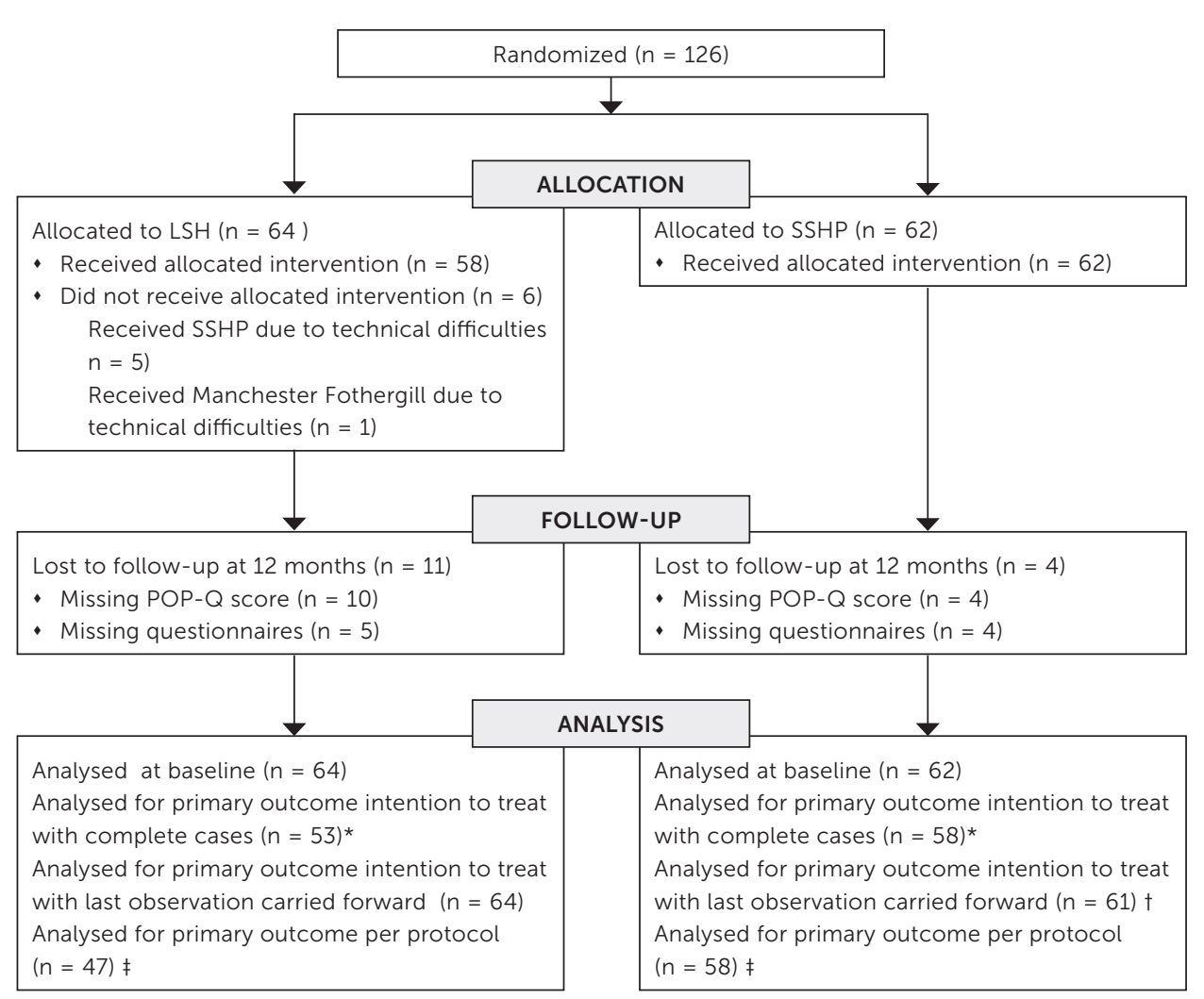

FIGURE 1. Flow of women through the study

* Intention to treat complete cases: only patients with complete follow-up (e.g. POP-Q and questionnaire) were analyzed according to allocation. Six patients allocated to LSH received a vaginal procedure and were analyzed in the laparoscopic group.

I Intention to treat with last observation carried forward: missing data was imputed with data at the six months follow-up visit if available $(n=12)$ or, in case these data is also missing, data of 6 weeks follow-up visit $(n=2)$. In one patient allocated to the SSHP, no follow-up data was available.

₹ Per protocol analysis: 6 patients did not received intended treatment. Excluded per protocol analysis: lost to follow-up at 12 months $(n=15)$ and major protocol deviations $(n=7)$.

\section{RESULTS}

In total, 126 women were randomly assigned to LSH $(n=64)$ or SSHP $(n=62)$ between August 2013 and September 2016. Figure 1 displays the flowchart of this study. Seven protocol deviations occurred. Five women received SSHP instead of LSH (crossovers). Reasons for crossover were intra-abdominal adhesions making mesh placement difficult $(n=2)$, excessive intra-abdominal fat tissue with impaired visualization of the promontory $(n=1)$, an enlarged uterus reaching up to the promontory $(n=1)$ and perforation of the vaginal wall during dissection $(n=1)$. In one woman allocated to $\mathrm{LSH}$, it was impossible to attach the mesh to the promontory due to extremely hard tissue. Consequently, a Manchester Fothergill procedure was performed. Finally, in one woman an endometrial polyp was removed during LSH. Histopathologic examination demonstrated endometrial carcinoma. Two months after surgery, a total laparoscopic hysterectomy was performed. Baseline characteristics and prolapse staging were comparable between groups (table 1).

In the SSHP group, there were significantly more additional anterior (SSHP: $n=61,98.4 \%$; LSH: $n=55,85.9 \%, p 0.010$ ), but not posterior vaginal wall repairs (SSHP: $n=14,22.6 \%$; LSH: $n=13,20.3 \%$ ). In the SSHP group, all anterior vaginal wall repair was performed by anterior colporrhaphy, while in the LSH group the majority of the anterior repair was performed laparoscopically $(n=44,80 \%$; vaginally $n=11,20 \%$ ). When looking at posterior repair, women in the SSHP group underwent posterior colporrhaphy $(n=12$, $86 \%)$ or perineorrhaphy $(n=2,14 \%)$. In the LSH group, posterior repair was performed laparoscopically $(n=5,38 \%)$, by posterior colporraphy $(n=5,38 \%)$ or by perineorraphy $(n=3,23 \%)$. Three associated tension-free vaginal tapes were placed, two after $L S H$ (3.1\%) and one (1.6\%) after SSHP

Table 2 presents the results on the primary outcome and the additional definitions of surgical failure. Regarding the primary outcome, LSH was non-inferior to SSHP: LSH $1.6 \%(n=1)$ versus SSHP $3.3 \%(n=2)$, difference $-1.7 \%(95 \% \mathrm{Cl}-7.1$ to 3.7$)$ for the ITT-LOCF approach. Non-inferiority of the LSH was also shown in the ITT- analysis with complete cases and the PP-analysis. The worst-case scenario did not lead to different conclusions. No difference was found in overall surgical failure, composite outcome of success and anatomical failure. Most anatomical failures occurred in the anterior compartment.

Table 3 provides details on the functional outcomes. After LSH, significantly more bothersome symptoms of overactive bladder (OAB) were reported (UDI-score LSH: 11 (interquartile range (IQR) 0-22); SSHP 0 (IQR 0-11), $\mathrm{p}=0.012$ ). De novo OAB occurred in 2 women (4.0\%) after LSH. However, $28 \%(14 / 50)$ of women after LSH who suffered already of $O A B$ before surgery reported persistent $O A B$ symptoms after surgery as compared to $13.5 \%(7 / 52)$ of women after SSHP.

Significantly more bothersome symptoms of faecal incontinence following LSH were reported (DDI-score LSH: 0 (IQR 0-17); SSHP: 0 (IQR 0-0), $p=0.017$ ). Persistent faecal incontinence was reported in 3.4\% (2/58) of women after SSHP, as compared to $10.2 \%$ (6/59) of women after LSH. De novo faecal incontinence was reported in 5 women (8.5\%) after LSH compared to 4 women (6.9\%) after SSHP. 
Table 1. Baseline characteristics and pelvic measurements of women. Values are numbers (percentages) unless stated otherwise.

\begin{tabular}{|c|c|c|}
\hline Characteristics & $\begin{array}{l}\text { Laparoscopic } \\
\text { sacrohysteropexy }(n=64)\end{array}$ & $\begin{array}{l}\text { Vaginal sacrospinous } \\
\text { hysteropexy }(n=62)\end{array}$ \\
\hline Mean (SD) age (years) & $61.08(9.8)$ & $60.76(10.7)$ \\
\hline Mean (SD) body mass index $\left(\mathrm{kg} / \mathrm{m}^{2}\right)$ & $26.6(3.4)$ & $26.6(2.9)$ \\
\hline \multicolumn{3}{|l|}{ Comorbidity: } \\
\hline Cardiovascular disease & $29(45.3)$ & $19(30.6)$ \\
\hline Respiratory disease & $4(6.3)$ & $4(6.5)$ \\
\hline Diabetes Mellitus & $3(4.7)$ & $4(6.5)$ \\
\hline Current smoker (self-reported) & $5(7.8)$ & $7(11.3)$ \\
\hline Median (range) number of vaginal deliveries & $2(1-10)$ & $2(1-5)$ \\
\hline Median (range) number of caesarean deliveries & $0(0-1)$ & $0(0-1)$ \\
\hline \multicolumn{3}{|l|}{$P O P-Q$ stage uterine prolapse $(\text { point } C)^{*}$ : } \\
\hline 2 & $45(70.3)$ & $39(62.9)$ \\
\hline 3 & $18(28.1)$ & $19(30.6)$ \\
\hline 4 & $1(1.6)$ & $4(6.5)$ \\
\hline \multicolumn{3}{|l|}{ POP-Q stage 2-4: } \\
\hline Anterior prolapse $(\mathrm{Ba} \geq-1)$ & $59(92.2)$ & $56(90.3)$ \\
\hline Posterior prolapse $(\mathrm{Ba} \geq-1)$ & 14 (22) & $16(25.8)$ \\
\hline \multicolumn{3}{|l|}{ Prolapse beyond hymen: } \\
\hline Apical (POP-Q C >0) & $30(46.6)$ & $28(45.2)$ \\
\hline Anterior (POP-Q Aa or $\mathrm{Ba}>0$ ) & $51(81)$ & $45(72.6)$ \\
\hline Posterior (POP-Q Ap or $\mathrm{Bp}>0$ ) & $2(3.2)$ & $3(4.8)$ \\
\hline \multicolumn{3}{|l|}{ Vaginal bulge symptoms: } \\
\hline Any & $56 / 62(90.3)$ & $56 / 59(94.9)$ \\
\hline Bothersome & $54 / 62(87.1) \dagger$ & $56 / 59$ (94.9) \\
\hline
\end{tabular}

$\mathrm{POP}-\mathrm{Q}=$ pelvic organ prolapse quantification

Percentages were calculated using non-missing data. All patients were analyzed as allocated.

* System involves quantitative measurements of various points of vaginal wall with hymen as reference point. Degree of prolapse of anterior vaginal wall (Aa and Ba), posterior vaginal wall (Ap and Bp) and uterus (C) is measured in centimeters above or proximal to hymen (negative number) of beyond or distal to the hymen (positive number), with plane of hymen defined as zero. Point A represents the descent of a measurement point 3 $\mathrm{cm}$ proximal to the hymen on the anterior ( $\mathrm{Aa}$ ) and posterior (Ap) vaginal wall. B is the most descended edge on the anterior $(\mathrm{Ba})$ and posterior $(\mathrm{Bp})$ vaginal wall.

POP-Q stage 2: most distal prolapse is between $1 \mathrm{~cm}$ above and $1 \mathrm{~cm}$ beyond hymen

POP-Q stage 3: most distal prolapse is $>1 \mathrm{~cm}$ beyond hymen, but no further than $2 \mathrm{~cm}$ less than total vaginal length;

POP-Q stage 4: total prolapse.

† Not all women reported bothersome prolapse symptoms at baseline. The questionnaire was provided after women consented to participate, therefore amount of bother as reported at outpatient clinic could differ.

Quality of life and sexual functioning were not different. In the laparoscopic group, $84 \%(42 / 50)$ of the women were sexually active as compared to $75.5 \%(40 / 53)$ in the vaginal group. Of the sexually active women, 13 out of 39 women $(33.3 \%$ ) of the SSHP group reported dyspareunia, which is almost three times as often compared to the laparoscopic group $(5 / 37,13.5 \%, p=0.042)$. De novo dyspareunia was found in respectively 5 women $(13.2 \%$, SSHP) and 3 women ( $8.1 \%$, LSH, not significant).
Table 2. Outcomes for pelvic organ prolapse at 12 month follow-up. Values are numbers (percentages) of women unless states otherwise.

\begin{tabular}{|c|c|c|c|}
\hline Outcomes & $\begin{array}{l}\text { Laparoscopic } \\
\text { sacrohysteropexy }\end{array}$ & $\begin{array}{l}\text { Vaginal sacrospinous } \\
\text { hysteropexy }\end{array}$ & Difference $(95 \% \mathrm{Cl})$ \\
\hline \multicolumn{4}{|l|}{ Primary outcome surgical failure* } \\
\hline ITT analysis with complete cases & $1 / 54(1.9 \%)$ & $2 / 58(3.4 \%)$ & $-1.6(-7.5$ to 4.3$)$ \\
\hline ITT analysis with LOCF & $1 / 64(1.6 \%)$ & $2 / 61(3.3 \%)$ & $-1.7(-7.1$ to 3.7$)$ \\
\hline Per protocol analysis & $1 / 47(2.1 \%)$ & $2 / 58(3.4 \%)$ & $-1.3(-7.6$ to 4.9$)$ \\
\hline \multicolumn{4}{|l|}{ Overall surgical failuret } \\
\hline ITT analysis with complete cases & $39 / 57(68.4 \%)$ & $37 / 58(63.8 \%)$ & $4.6(-12.7$ to 21.9$)$ \\
\hline ITT analysis with LOCF & $42 / 64(65.6 \%)$ & $38 / 61(62.3 \%)$ & $3.3(-13.5$ to 20.2$)$ \\
\hline Per protocol analysis & $35 / 50(70 \%)$ & $37 / 58(63.8 \%)$ & $6.2(-11.5$ to 23.9$)$ \\
\hline \multicolumn{4}{|l|}{ Composite outcome success $\ddagger$} \\
\hline ITT analysis with complete cases & $44 / 55(80.0 \%)$ & $48 / 58(82.8 \%)$ & $-2.8(-17.1$ to 11.6$)$ \\
\hline ITT analysis with LOCF & $53 / 64(82.8 \%)$ & $51 / 61(83.6 \%)$ & $-0.8(-13.9$ to 12.3$)$ \\
\hline Per protocol analysis & $38 / 49(77.6 \%)$ & $48 / 58(82.8 \%)$ & $-5.2(-20.4$ to 10.0$)$ \\
\hline \multicolumn{4}{|l|}{ Anatomical failure $\mathbb{\Xi}$} \\
\hline Overall anatomical failure & $35 / 55(63.6 \%)$ & $36 / 58(62.1 \%)$ & $1.6(-16.3$ to 19.4$)$ \\
\hline Apical compartment & $2 / 55(3.6 \%)$ & $2 / 58(3.4 \%)$ & $0.2(-6.6$ to 7.0$)$ \\
\hline Anterior compartment & $28 / 55(50.9 \%)$ & $33 / 58(56.9 \%)$ & $-6.0(-24.3$ to 12.4$)$ \\
\hline Posterior compartment & $10 / 55(18.2 \%)$ & $4 / 58(6.9 \%)$ & $11.3(-0.8$ to 23.4$)$ \\
\hline \multicolumn{4}{|l|}{ Prolapse beyond hymen } \\
\hline Apical (POP-Q C >0) & $0 / 64(0 \%)$ & $1 / 61(1.6 \%)$ & $-1.6(-4.8$ to 1.5$)$ \\
\hline Anterior (POP-Q Ba > 0) & $6 / 64(9.4 \%)$ & $5 / 61(8.2 \%)$ & $1.2(-8.7$ to 11.1$)$ \\
\hline Posterior (POP-Q Bp >0) & $0 / 64(0 \%)$ & $0 / 61(0 \%)$ & n.a. \\
\hline \multicolumn{4}{|l|}{ Repeat surgery } \\
\hline Overall repeat surgery & $2 / 64(3.1 \%)$ & $3 / 61(4.9 \%)$ & $-1.8(-8.7$ to 5.1$)$ \\
\hline Apical compartment & $0 / 64(0 \%)$ & $2 / 61(3.3 \%)$ & $-3.3(-7.7$ to 1.2$)$ \\
\hline Anterior compartment & $2 / 64(3.1 \%)$ & $1 / 61(1.6 \%)$ & $1.5(-3.1$ to 1.6$)$ \\
\hline Posterior compartment & $0 / 64(0 \%)$ & $0 / 61(0 \%)$ & n.a. \\
\hline Primary surgery different site ** & 0/64 (0\%) & $0 / 61(0 \%)$ & n.a. \\
\hline \multicolumn{4}{|c|}{ Surgery for non-prolapsed conditions } \\
\hline Anti-incontinence & $4 / 64(6.3 \%)$ & $2 / 61(3.3 \%)$ & $3.0(-4.5$ to 10.4$)$ \\
\hline Hysterectomy & $1 / 64(1.6 \%)$ & $0 / 61(0 \%)$ & n.a. \\
\hline \multirow{2}{*}{\multicolumn{4}{|c|}{$\begin{array}{l}\text { ITT = intention to treat; } L O C F=\text { last observation carried forward; } P O P-Q=\text { pelvic organ prolapse quantification } \\
\text { Percentages were calculated using non-missing data. }\end{array}$}} \\
\hline & & & \\
\hline \multirow{5}{*}{\multicolumn{4}{|c|}{$\begin{array}{l}\text { Percentages were calculated using non-missing data. } \\
\text { * Recurrent apical prolapse POP-Q } \geq 2 \text { with bothersome symptoms or repeat surgery or pessary for } \\
\text { apical prolapse } \\
\dagger \text { Prolapse POP-Q stage } \geq 2 \text { (any compartment) or repeat surgery or pessary use } \\
\text { ‡ No prolapse beyond hymen (any compartment), absence of bothersome bulge symptoms, and no repeat } \\
\text { surgery or pessary use }\end{array}$}} \\
\hline & & & \\
\hline & & & \\
\hline & & & \\
\hline & & & \\
\hline $\begin{array}{l}\text { I Prolapse POP-Q stage } \geq 2 \\
\text { IITT with LOCF }\end{array}$ & & & \\
\hline & & & \\
\hline
\end{tabular}


We found a relatively high crossover rate from LSH to SSHP. Nevertheless, all gynaecologists participated in this trial were fully trained in advanced laparoscopic urogynaecology procedures and performed LSH at a regular base prior to the start of this study. Reasons for crossover were adhesions, excessive intra-abdominal tissue, an enlarged uterus and perforation of the vaginal wall during dissection.

Due to different definitions of the primary outcome and the usage of different questionnaires to analyse functional outcome and quality of life, it is difficult to make a comparison with other studies [21]. In addition, studies on the outcome of LSH are limited, typically pooling data with outcomes after the sacrocolpopexy (either for vault prolapse or concomitant (subtotal) hysterectomy), making it hard to separate outcomes.

There are however several RCTs that compare SSHP with vaginal hysterectomy [15,17]. In these, SSHP is as effective as vaginal hysterectomy for the treatment of uterine prolapse. Our findings are in line with those studies though we observed a higher overall surgical failure after SSHP at one-year follow-up (62.3\% versus 51\%) [15]. This might be caused by a higher incidence of prolapse of the anterior compartment in our study group (56.9\% versus $47 \%$ ), whereas the rates of concomitant anterior colporrhaphy were comparable $(98.4 \%$ and $97 \%)$. This may be attributed to the large variation in surgical technique of anterior colporrhaphy, though other factors may play a role as well [36]. Compared to the RCT of Dietz et al. we found less anatomical recurrences of the apical compartment (3.4\% versus $21 \%)$ after SSHP [17]. The latter number is rather high for SSHP $[15,16,37]$. In the RCT of Dietz et al, performed between 2001 and 2005, SSHP was perhaps a relatively novel technique for those surgeons. The differences between anatomical recurrence might be explained by more experience nowadays.

In a prospective study by Price et al, one surgical failure (2\%) of the apical compartment was observed at 10-week follow-up, compared to $1.9 \%$ in our study [38]. In a prospective observational study 3 women (2\%) required reoperation for apical support for symptomatic prolapse at a mean follow-up of 2.1 years [39]. In our study, no reoperation of the apical compartment was observed at a follow-up of 12 months, probably due to the shorter follow-up. Furthermore, our findings are mainly in line with a randomised study comparing LSH to vaginal hysterectomy [40]. However, we found lower reoperation rates of the apical compartment at one-year follow-up $(0 \%$ versus $6 \%$ ). Differences in sample size (37 versus 59 ) and subtle differences in surgical protocol might explain this finding

\section{STRENGTHS AND LIMITATIONS}

Strengths of this study are the randomised multicentre design and the large sample size. Also, prior to first enrolment, all study centres participated in a masterclass, where both surgeries were discussed in detail and a standardized approach was demonstrated. The primary outcome was defined as prolapse of the apical compartment in combination with bothersome bulge symptoms or repeat surgery or pessary use for recurrent prolapse. It is known that treatment success varies widely depending on the definition of surgical success. However, definitions of treatment success that require patient reported outcome are more clinically relevant and meaningful to the patient than those that include anatomical criteria only [21].

We acknowledge some limitations. First, our findings are based on a short-term followup. However, a recent cohort study demonstrated that the highest risk for undergoing repeat surgery for POP is within the first year [41]. Another limitation is the relatively high loss to follow-up in the laparoscopic group, with an eventual number of women with data available lower than what was required ( $<55$ women). Two women withdrew the informed consent due to intercurrent illness, and nine did not report at 12 months, hence observations at 6 months were used. At that time, no anatomical recurrence of the apical compartment was observed.

\section{INTERPRETATION}

This study provides evidence that on the short-term LSH is as effective as SSHP for the treatment of uterine prolapse. The subtle differences in secondary outcomes may help in the process of shared-decision making and choose the optimal surgical route for a specific patient. Symptoms of OAB seem to improve more after SSHP as compared to LSH. Furthermore, persistent faecal incontinence was reported more frequent after LSH compared to SSHP. Since treatment options are limited and associated with sideeffects, faecal incontinence is a devastating outcome [42,43]. Dyspareunia occurred more frequently after SSHP.

There is a wide variation in LSH techniques on several key points such as level of dissection, mesh type and tension of the mesh [44]. This variation could play a role in both anatomical and functional results. Well-designed trials regarding the procedure are needed to provide evidence for the best surgical technique. 


\section{CONCLUSIONS}

Twelve months after the index procedure, LSH was non-inferior to SSHP for bothersome bulging/protrusion symptoms and/or therapy for recurrent prolapse in the middle compartment.

Following $\mathrm{LSH}$, bothersome $\mathrm{OAB}$ and faecal incontinence were more frequent, yet dyspareunia was less frequent.

\section{REFERENCES}

1. Fritel $X$, Varnoux $N$, Zins $M$, Breart $G$, Ringa $V$. Symptomatic pelvic organ prolapse at midlife, quality of life, and risk factors. Obstet Gynecol. 2009;113(3):609

2. Wu JM, Matthews CA, Conover MM, Pate V. Jonsson Funk M. Lifetime risk of stress urinary incontinence or pelvic organ prolapse surgery. Obstet Gynecol 2014;123(6):1201-6

3. Dieter AA, Wilkins MF, Wu JM. Epidemiological trends and future care needs for pelvic floor disorders. Curr Opin Obstet Gynecol 2015;27(5):380-4

4. Gutman R, Maher C. Uterine-preserving POP surgery. Int Urogynecol J 2013:24(11):1803-13

5. DeLancey JO. Anatomic aspects of vaginal eversion after hysterectomy. Am J Obstet Gynecol 1992;166(6):1717-24

6. Madsen AM, Raker C, Sung VW. Trends in hysteropexy and apical support for uterovaginal prolapse in the united states from 2002 to 2012. Female Pelvic Med Reconstr Surg 2017;23(6):365-

7. Jha S, Cutner A, Moran P. The UK national prolapse survey: 10 years on. Int Urogynecol J 2018;29(6):795-801

8. Wu MP, Long $\mathrm{CY}$, Huang $\mathrm{KH}$, Chu CC, Liang CC, Tang $\mathrm{CH}$. Changing trends of surgical approaches for uterine prolapse: an 11-year population based nationwide descriptive study. Int Urogynecol J 2012;23(7):865-72.

9. Detollenaere RJ, den Boon J, Kluivers KB, Vierhout $M E$, van Eijndhoven HW. Surgical management of pelvic organ prolapse and uterine descent in the Netherlands. Int Urogynecol J 2013;24(5):781-8

10. Korbly NB, Kassis NC, Good MM, Richardson ML, Book NM, Yip S et al. Patient preferences for uterine preservation and hysterectomy in women with pelvic organ prolapse. Am J Obstet Gynecol 2013;209(5):470

11. Van IJsselmuiden MN, Detollenaere RJ, Gerritse MBE, Kluivers KB, Bongers MY, van Eijndhoven HWF. Dutch women's attitude towards hysterectomy and uterus preservation in surgical treatment of pelvic organ prolapse. Eur J Obstet Gynecol Reprod Biol 2018;220:79-83

12. Frick $A C$, Barber MD, Paraiso MF, Ridgeway B, Jelovsek JE, Walters MD. Attitudes toward hysterectomy in women undergoing evaluation for uterovaginal prolapse. Female Pelvic Med Reconstr Surg 2013;19(2):103-9

13. Bradley S, Gutman RE, Richter LA. Hysteropexy. an option for the repair of pelvic organ prolapse. Curr Urol Rep 2018;19(2):15

14. Meriwether KV, Antosh DD, Olivera CK, Kim-Fine S, Balk EM, Murphy M et al. Uterine preservation vs hysterectomy in pelvic organ prolapse surgery: a systematic review with meta-analysis and clinical practice guidelines. Am J Obstet Gynecol 2018;219(2):129-46

15. Detollenaere RJ, den Boon J, Stekelenburg J, IntHout J, Vierhout ME, Kluivers KB et al. Sacrospinous hysteropexy versus vaginal hysterectomy with suspension of the uterosacral ligaments in women with uterine prolapse stage 2 or higher: multicentre randomised non-inferiority trial. BMJ 2015;351:h3717

16. Schulten SFM, Detollenaere RJ, Stekelenburg J, IntHout J, Kluivers KB, van Eijndhoven HWF. Sacrospinous hysteropexy versus vaginal hysterectomy with uterosacral ligament suspension in women with uterine prolapse stage 2 or higher: observational follow-up of a 
multicentre randomized trial. BMJ 2019;366:15149

17. Dietz V, van der Vaart CH, van der Graaf $Y$, Heintz P, Schraffordt Koops S. One-year followup after sacrospinous hysteropexy and vaginal hysterectomy for uterine descent: a randomized study. Int Urogynecol J 2010;21(2):209-16

18. Zacche MM, Mukhopadhyay S, Giarenis I. Trends in prolapse surgery in England. Int Urogynecol J 2018;29(11):1689-95

19. van IJsselmuiden MN, Coolen AWM, Detollenaere RJ, den Boon J, Bongers MY, van de Pol G et al. Hysteropexy in the treatment of uterine prolapse stage 2 or higher: a multicenter randomized controlled non-inferiority trial comparing laparoscopic sacrohysteropexy with vaginal sacrospinous hysteropexy (LAVA-trial, study protocol). BMC Women's Health 2014, 14:112.

20. Bump RC, Mattiasson A, Bø K, Brubaker LP DeLancey JO, Klarskov $P$ et al. The standardization of terminology of female pelvic organ prolapse and pelvic floor dysfunction. Am J Obstet Gynecol 1996;175:10-7.

21. Barber MD, Brubaker L, Nygaard I, Wheeler TL $2^{\text {nd }}$, Schaffer J, Chen $Z$ et al. Defining surgical success after surgery for pelvic organ prolapse. Obstet Gynecol 2009;114:600-9.

22. Ware JE, Kosinksi M, Keller SD. SF-36 physical and mental component summary measures - a user's manual. 1994. New England Medical Center, The Health Institute.

23. van der Vaart $\mathrm{CH}$, de Leeuw JR, Roovers JP, Heintz AP. Measuring health-related quality of life in women with urogenital dysfunction: the urogenital distress inventory and incontinence impact questionnaire revisited. Neurourol Urodyn 2003;22:97-104.

24. Roovers JP, van der Bom JG, van der Vaart $\mathrm{CH}$, Heintz AP. Prediction of findings at defecography om patients with genital prolapse. $\mathrm{Br} \mathrm{J}$ Obstet Gynaecol 2005;112:1547-53.

25. Lamers LM, Stalmeier PF, McDonnell K, Krabbe PF, van Busschbach JJ. Measuring the quality of life in economic evaluations: the Dutch EQ-5D tariff. Ned Tijdschr Geneeskd 2005;149:1574-8

26. Schweitzer KJ, de Jong M, Milani AL. Prolaps en seks: hoe meten we de relatie. Ned Tijdschr Obst Gyn 2008;121:79-82

27. Abdullah B, Nomura J, Moriyama S, Huang T, Tokiwa S, Togo M. Clinical and urodynamic assessment in patients with pelvic organ prolapse before and after laparoscopic sacrocolpopexy. Int Urogynecol J 2017;28:1543-9

28. Johnson JR, High RA, Dziadek O, Ocon A, Muir TW, Xu J et al. Overactive bladder symptoms after pelvic organ prolapse repair. Female Pelvic Med Reconstr Surg 2019 [Epub ahead of print]

29. Kummeling MT, Rietbergen JB, Withagen MI, Mannaerts GH, van der Weiden RM. Sequential urodynamic assessment before and after laparoscopic sacrocolpopexy. Acta Obstet Gynecol Scan 2013;92(2):172-7

30. Rogowkski A, Bienkowski P, Tosiak A, Jerzak M, Mierzejewski P, Baranowski W. Mesh retraction correlates with vaginal pain and overactive bladder symptoms after anterior vaginal mesh repair. Int Urogynecol J 2013;24(12):2087-92

31. Nygaard IE, McCreery R, Brubaker L, Connolly A, Cundiff $G$, Weber AM et al. Abdominal sacrocolpopexy: a comprehensive review. Obstet Gynecol 2004;104(4):805-23

32. Christmann-Schmid C, Koerting I, Ruess E, Faehnle I, Krebs J. Functional outcome after laparoscopic nerve-sparing sacrocolpopexy: a prospective cohort study. Acta Obstet Gynecol Scand 2018;97(6):744-50

33. Richardson ML, Elliot CS, Sokol ER. Posterior compartment prolapse: a urogynecology perspective. Urol Clin North Am 2012;39(3):361-9

34. Detollenaere RJ, Kreuwel IA, Dijkstra JR, Kluivers KB, van Eijndhoven HW. The impact of sacrospinous hysteropexy and vaginal hysterectomy with suspension of the uterosacral ligaments on sexual function in women with uterine prolapse: a secondary analysis of a randomized comparative study. J Sex Med 2016:13(2):213-9

35. Morgan DM, Rogers MA, Huebner M, We JT, Delancey JO. Heterogeneity in anatomic outcome of sacrospinous ligament fixation for prolapse: a systematic review. Obstet Gynecol 2007:109:1424-33.

36. Lensen EJ, Stoutjesdijk JA, Withagen MI Kluivers KB, Vierhout ME. Technique of anterior colporrhaphy: a Dutch evaluation. Int Urogynecol J 2011;22(5):557-61.

37. Schraffordt DV, Koops SE, Vaart CH. Vaginal surgery for uterine descent: which options do we have? A literature review. Int Urogynecol J 2008:20(3):349-56

38. Price N, Slack A, Jackson SR. Laparoscopic hysteropexy: the initial results of a uterine suspension procedure for uterovaginal prolapse. BJOG 2010;117(1):62-8

39. Rahmanou P, White B, Price N, Jackson S. Laparoscopic hysteropexy: 1- to 4-year followup of women postoperatively. Int Urogynecol J 2014:25(1):131-8

40. Rahmanou P, Price N, Jackson SR. Laparoscopic hysteropexy versus vaginal hysterectomy for the treatment of uterovaginal prolapse: a prospective randomized pilot study. Int Urogynecol J 2015;26(11):1687-94

41. Løwenstein E, Møller LA, Laigaard J, Gimbel H. Reoperation for pelvic organ prolapse: a Danish cohort study with 15-20 years'follow-up. Int Urogynecol J 2018;29(1):119-124

42. Saldana Ruiz N, Kaiser AM. Fecal Incontinence Challenge and solutions. World J Gastroenterol 2017;7:23(1):11-24

43. Wald A. Diagnosis and management of Faecal Incontinence. Curr Gastroenterol Rep 2018;20:9.

44. Van IJsselmuiden MN, Kerkhof MH, Schellart RP, Bongers MY, Spaans WA, van Eijndhoven HWF. Variation in the practice of laparoscopic sacrohysteropexy and laparoscopic sacrocolpopexy for the treatment of pelvic organ prolapse: a Dutch survey. Int Urogynecol J 2015;26(5):757-64 
DYNAMIC MAGNETIC RESONANCE IMAGING TO QUANTIFY PELVIC ORGAN MOBILITY AFTER

TREATMENT FOR UTERINE DESCENT:

DIFFERENCES BETWEEN SURGICAL PROCEDURES

M.N. van IJsselmuiden

P. Lecomte-Grosbras

J.F. Witz

M. Brieu

M. Cosson

H.W.F. van Eijndhoven 


\section{ABSTRACT}

\section{INTRODUCTION}

Pelvic organ mobility is defined as the displacement of pelvic organs between rest and maximal straining. We hypothesized that pelvic organ mobility after vaginal sacrospinous hysteropexy (SSHP) is increased as compared to other surgeries for uterine descent, which might contribute to the high occurrence of postoperative cystocele after this surgery. Pelvic organ mobility and the vaginal axes after SSHP are compared with other surgical procedures for uterine descent: vaginal hysterectomy with uterosacral suspension (VH) and laparoscopic sacrohysteropexy (LSH).

\section{METHODS}

In this prospective pilot study, 15 women were included (5 of each procedure). Six months postoperative, POP-Q examination and dynamic MRI were performed and questionnaires were filled out regarding prolapse complaints. Pelvic organ mobility on MRI was defined as vertical displacement of pelvic organs at rest and maximal straining. The displacements and angles were measured using an image registration method. Furthermore, the angle of displacement of cervix/vaginal vault and vaginal axes were assessed

\section{RESULTS}

No anatomical recurrences of pelvic organ prolapse were found. No difference in pelvic organ mobility was demonstrated. After $\mathrm{VH}$, a more posterior position of the upper vagina was found compared to SSHP and LSH.

\section{CONCLUSION}

Based on these data, the higher recurrence risk in the anterior compartment after SSHP cannot be explained. Larger sample sizes, studying women with recurrence or de novo cystocele after SSHP or using an upright MRI scanner would be of interest to further assess the relation between pelvic organ mobility and the occurrence of anterior vaginal wall prolapse.

\section{INTRODUCTION}

Worldwide, vaginal hysterectomy $(\mathrm{VH})$ with uterosacral suspension of the vaginal vault is the most important treatment for symptomatic uterovaginal prolapse [1]. However, a high proportion of women with prolapse symptoms prefer uterine preservation instead of hysterectomy [2,3]. As a consequence, these preserving procedures are becoming more popular [4,5]. Recent studies have demonstrated that suspension of the cervix to the sacrospinous ligament, the so called vaginal sacrospinous hysteropexy (SSHP), is as effective as the $\mathrm{VH}[6,7]$.

The risk for recurrent prolapse of the anterior vaginal wall after SSHP is often discussed with incidences ranging from 5.8 to $21.3 \%[8,9]$. A recent randomized controlled trial comparing SSHP to $\mathrm{VH}$ demonstrated more anterior vaginal wall recurrences after SSHP $(40 \%$ vs $36 \%)$, but more posterior vaginal wall recurrences after $\mathrm{VH}(18 \%$ vs $5 \%)$ [6]. As for anatomic recurrence after SSHP, the cervix generally remains well fixed to the sacrospinous ligament, but the weak point is supposed to be the anterior compartment [10]. It is hypothesized that the high rates of recurrence in the anterior vaginal wall after SSHP may be related to the previously incurred damage of muscular supports (e.g. levator ani muscle injury, reduced muscle strength), the change in vaginal axis to a more posterior and horizontal position or a combination of these two $[9,11]$

Imaging has become an important complementary tool in the assessment of pelvic floor disorders. Magnetic resonance imaging (MRI) can simultaneously evaluate all the compartments of the pelvic floor, and provide information about muscles and ligaments. Furthermore, dynamic MRI of the pelvic floor allows detailed functional evaluation of the pelvic floor [12].

Pelvic organ mobility is defined as the displacement of pelvic organs between rest and maximal straining. Studying pelvic organ mobility after surgery for uterine prolapse is of interest, because it can provide more insight in the cause of recurrence of anterior vaginal wall prolapse after SSHP. It is unknown how mechanical forces after pelvic organ prolapse (POP) surgery change and whether or not they contribute to this recurrence. Numerical simulation methods have been developed to analyse pelvic organ mobility and to evaluate the role of support structure on mobility in general and in case of POP, or it can be used to simulate surgery [13-15]. These methods might also help to reveal the distribution of mechanic forces on pelvic structures after surgery and compare these forces between surgical procedures. With patient-specific analysis and simulations the process of decision making in case of surgical intervention 
could be improved. However, all these numerical methods have not been validated by quantitative measurements especially after POP surgery.

We hypothesized that, after SSHP, pelvic organ mobility of the cervix is decreased due to fixation of the cervix. Therefore, more pressure on the anterior vaginal wall occurs, resulting in an increase in pelvic organ mobility of the anterior vaginal wall. This might contribute to the occurrence of postoperative cystocele. In addition, vaginal axis and its change between rest and maximal straining is assessed, because this might contribute to the recurrence of anterior vaginal wall prolapse as well. In this study, the mobility of pelvic organs and the vaginal axis after vaginal SSHP are compared with $\mathrm{VH}$ with uterosacral suspension of the vaginal vault and laparoscopic sacrohysteropexy (LSH).

\section{MATERIALS AND METHODS}

This prospective observational pilot study was performed in the Isala hospital (Zwolle, the Netherlands) from November 2014 to May 2017. After approval from the medical ethics committee, eligible women were asked to participate in this study. Three different surgical procedures for uterine descent were analyzed: vaginal sacrospinous hysteropexy (SSHP), laparoscopic sacrohysteropexy (LSH) and vaginal hysterectomy $(\mathrm{VH})$. All women who recently underwent one of those procedures as a primary treatment for uterine descent were eligible to participate in this study. Exclusion criteria were factors that precludes MRI interpretation (e.g. prosthetic hip), contraindications for MRI (e.g. claustrophobia), patients with neurological disease affecting the pelvic floor or with previous pelvic floor surgery and patients who were not able to maintain Valsalva maneuver for at least 10 seconds (e.g. due to pulmonary problems). A total of 15 women were enrolled in this study (5 of each procedure). All women gave written informed consent. Six months after surgery, women underwent gynecological examination and dynamic MRI, and filled out validated questionnaires.

\section{SURGICAL TECHNIQUES}

SSHP was carried out by fixing the posterior side of the cervix to the sacrospinous ligament using two permanent sutures (Prolene 1/0, Ethicon Inc, Sommerville, NJ, USA). The sutures were placed unilaterally to the right sacrospinous ligament at least $2 \mathrm{~cm}$ from the ischial spine using a needle driver under direct visualization. Additional anterior or posterior vaginal wall repair was performed if indicated.LSH was performed using 4 laparoscopic ports (umbilical, suprapubic, 2 lateral ports). The peritoneum over the sacral promontory was incised and the broad ligament at the level of the cervicouterine junction was opened bilaterally. The arms of a bifurcated polypropylene mesh were introduced in these windows of the broad ligament, the mesh was attached to the anterior and posterior side of the cervix using permanent sutures. Afterwards, the mesh was attached to the sacral promontory using titanium staples. Afterwards, additional anterior and/or posterior colporrhaphy was performed if indicated.

Regarding $\mathrm{VH}$, the vaginal wall around the cervix was circumcised and, after bowel and bladder dissection, the anterior and posterior peritoneum were opened. The uterosacral ligaments were ligated and transected. Next, the uterus was released in several steps using clamps and sutures. After removing the uterus, the peritoneum was closed with absorbable sutures. Vault suspension was performed by suspension of the uterosacral ligaments. Such suspension involves the attachment of the uterosacral ligaments to the vaginal vault, thereby restoring normal support to the apical compartment. Concomitant anterior or posterior vaginal wall repair were performed afterwards if indicated.

\section{GYNECOLOGICAL EXAMINATION}

All women underwent gynecological examination, which includes vaginal inspection in $45^{\circ}$ semi-upright position for staging prolapse of the anterior, apical and posterior compartment using the Pelvic Organ Prolapse Quantification system (POP-Q) [16]. Maximum prolapse was demonstrated and identified by performing a Valsalva maneuver while each vaginal wall is individually exposed.

\section{QUESTIONNAIRES}

To assess the presence and discomfort of prolapse symptoms, women were asked to fill out a validated questionnaire. This questionnaire covers quality of life (Short Form-36, EQ-5D), urogenital and defecation symptoms (UDI, DDI, IIQ) and sexual dysfunction (PISQ-12) [17-21]. Furthermore, the questionnaire contained several questions with respect to parity, mode of delivery and BMI.

\section{DYNAMIC MRI}

All women underwent MRI in the supine position using a 1.5T MR scanner (Ingenia, Philips Healthcare, Best, the Netherlands) with an anterior coil centered low on the pelvis for better signal reception. Women were asked not to void their bladder one hour before examination, to ensure a moderate filled bladder. No intravenously administered contrast was used. Prior to the MRI, the rectum was filled with an aqueous gel (200 ml) with the patient in left lateral decubitus position, using a rectal catheter. Furthermore, 
Image at time $\mathrm{t}_{0}$

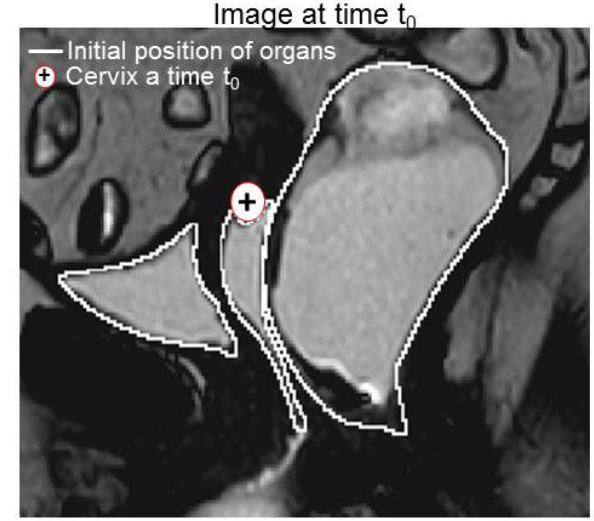

FIGURE 1 Assessing pelvic organ mobilty using MRI The white contours demonstrate the pelvic organs at rest The blue contours illustrate the position of these organs during straining. Pelvic organ mobility is the difference between the position at rest and during straining (in $\mathrm{mm}$ ) of these organs.

Three areas of the vagina were more particularly analyzed to compare mobility of each surgical procedures: anterior vagina (AV), posterior vagina (PV) and cervix/vaginal vault (figure 2). On each area, the mean vertical displacement was quantified in pixel (and converted in millimeters $(\mathrm{mm})$ ) by calculating the difference in position of these points in rest and during straining. Furthermore, the angle of displacement of the cervix/ vaginal vault was also assessed.
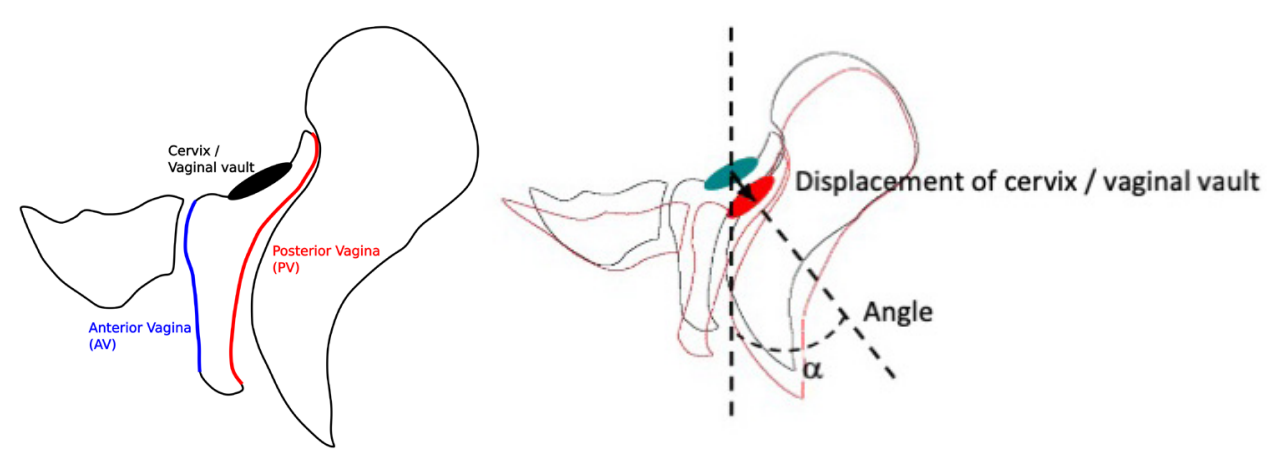
analyzing has been published previously [23]. This protocol was used to determine the displacement field on the contour of bladder, vagina and rectum as shown in figure 1.
FIGURE 2. a) Areas to quantify pelvic organ mobility in sagittal plane. Anterior vaginal wall (AV), posterior vaginal wall (PV) and cervix/vaginal vault were assessed. b) Angle of displacement of cervix/vaginal vault in sagittal plane. 
Moreover, each patient is applying loading more or less progressively, to compare mobility at same time or level of straining, a progress of the straining has been defined as a percentage calculated in time of straining

$$
\text { \%of strain }=\frac{t-t_{0}}{t_{\max }-t_{0}}
$$

Where $\%$ of strain is the percentage of progress of the loading time $t$ before the maximum strain, $t_{0}$ is the time of the beginning of the straining and $t_{\max }$ the time at maximum straining of the dynamic sequence. Figure 3 provides an example of the determination of pelvic organ mobility. In this sagittal plane, the organs are contoured at rest (in white) and during straining, the color bar corresponding to the displacement intensity on the contour of organs at 4 levels of straining.

\section{VAGINAL AXES}

To compare vaginal axes at rest, the vagina was separated into three distinct regions: upper, middle and lower vagina $[24,25]$. The upper region was defined as a straight line drawn from the anterior to the posterior fornix or from the anterior to the posterior aspect of the vaginal cuff in case of hysterectomy. The anterior vaginal wall (from introitus to anterior vaginal fornix or vaginal cuff) was divided in half, the proximal portion was defined as middle vaginal region, the distal portion was defined as lower vaginal region. Straight lines were drawn to fit both of these regions.

To correct for variations in pelvic inclination and to allow standardize measurements within the pelvis, the vaginal axis was measured as the angle between the vaginal axis and the pelvic inclination correction system (PICS line) [26]. This line is obtained by rotating a line from the inferior point of the pubic symphysis to the junction between the fifth lumbar vertebra and first sacral coccygeal bone (sacro-coccygeal inferior pubic point (SCIPP) line) by 34 degrees in a clockwise direction. For each vaginal region, vaginal axis was measured using this PICS line.

\section{STATISTICS}

All statistical analyses were performed with SPSS for Windows (version 24.0.0.1). Because the small number of participants in each group, data was assumed not being normally distributed. Consequently, medians and interquartile ranges were used as measures of central tendency and dispersion, respectively. Comparisons of continuous variables between the three surgeries were calculated using Kruskal Wallis tests, or, in case of two groups, Mann Whitney-U tests. Categorical variables were compared using Chi-square tests. P-values below 0.05 were considered to be significant.
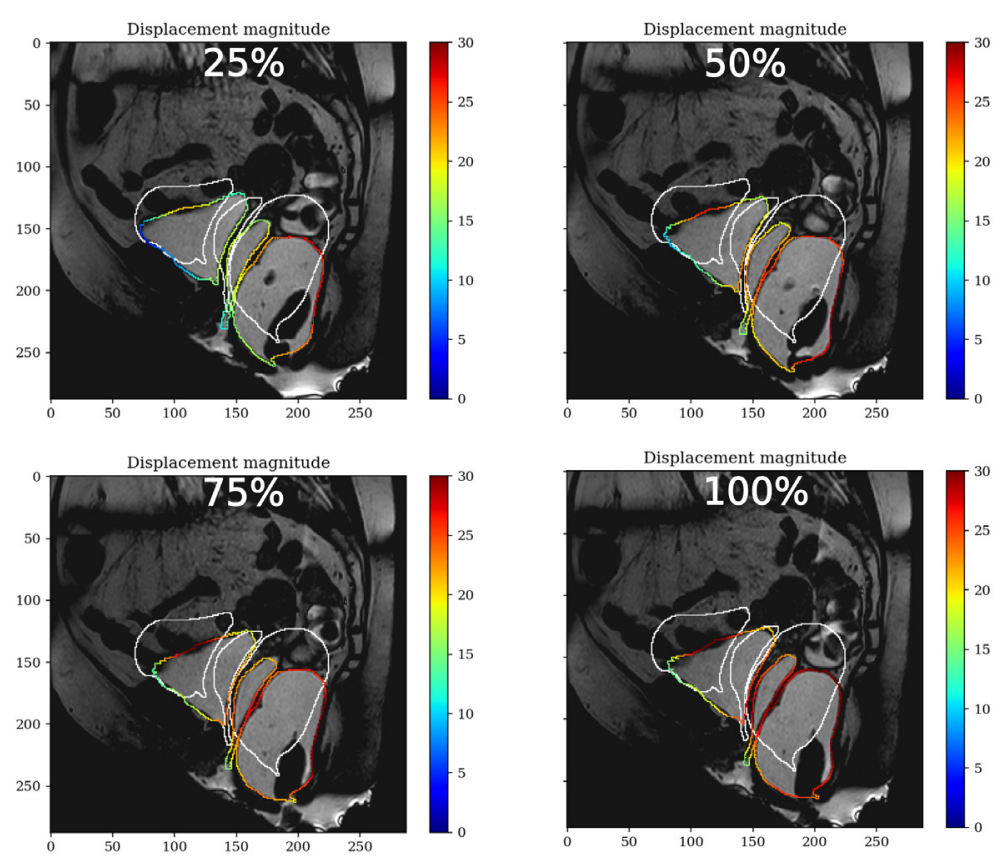

FIGURE 3. Vertical displacement field in $\mathrm{mm}$ on contour of pelvic organs at different percentage of maximum straining $(25 \%, 50 \%, 75 \%$ and $100 \%)$

\section{RESULTS}

Baseline characteristics were similar between the groups (table 1) with a median time between surgery and MR imaging of 31 weeks (LSH and VH group) and 33 weeks (SSHP group). Furthermore, concomitant surgeries were comparable between the three groups. The number of sexually active women was higher in the LSH and VH group $(n=4,80 \%)$ as compared to the SSHP group ( $n=1,33.3 \%$ ). However, only three out of five women of the SSHP group filled out the questionnaire regarding sexual functioning. In all groups, no anatomical recurrences of pelvic organ prolapse were found. Postoperative POP-Q assessment was comparable between the groups (table 1). 
Table 1. Characteristics of women. Values are numbers (percentages) unless stated otherwise.

\begin{tabular}{|c|c|c|c|c|}
\hline Characteristics & $\operatorname{SSHP}(n=5)$ & LSH (n=5) & $\mathrm{VH}(\mathrm{n}=5)$ & p-value* \\
\hline Median (IQR) age (years) & $62(57-68)$ & $68(48-70)$ & $57(50-68)$ & 0.690 \\
\hline Median (IQR) body mass index & $25.7(23.5-27.5)$ & $24.5(21.4-27.7)$ & $25.5(24.3-28.8)$ & 0.482 \\
\hline Median (IQR) parity & $3(2-6)$ & $3(2-6)$ & $3(2-4)$ & 0.815 \\
\hline \multicolumn{5}{|l|}{ Concomitant surgery } \\
\hline Anterior colporrhaphy & $4(80)$ & $3(60)$ & $5(100)$ & 0.287 \\
\hline Posterior colporrhaphy & $1(20)$ & $1(20)$ & $2(40)$ & 0.711 \\
\hline $\begin{array}{l}\text { Median time (IQR) between surgery } \\
\text { and MRI (weeks) }\end{array}$ & $33(31-39)$ & $31(27-44)$ & $31(28-34)$ & 0.606 \\
\hline Sexual active & $1(33.3)$ & $4(80)$ & $4(80)$ & 0.307 \\
\hline \multicolumn{5}{|c|}{ Preoperative POP-Q assessment (mean (SD)) } \\
\hline Аа & $0.8(2.4)$ & $-0.2(0.4)$ & $-0.2(0.8)$ & \\
\hline $\mathrm{Ba}$ & $1.0(2.3)$ & $1.2(1.3)$ & $1.4(0.9)$ & \\
\hline c & $-0.4(3.4)$ & $0.6(0.5)$ & $0.0(2.2)$ & \\
\hline gh & $3.4(0.5)$ & $4.2(0.8)$ & $4.6(0.5)$ & \\
\hline $\mathrm{pb}$ & $3(1.4)$ & $3.2(1.1)$ & $3.2(0.4)$ & \\
\hline TVL & $9.2(0.8)$ & $9.2(0.4)$ & $9.3(1.0)$ & \\
\hline Ap & $-1.6(1.5)$ & $-2.6(0.5)$ & $-2.6(0.5)$ & \\
\hline Bp & $-1.8(1.6)$ & $-2.6(0.5)$ & $-2.4(0.9)$ & \\
\hline D & $-4.2(3.6)$ & $-5(2.1)$ & $-7.5(0.6)$ & \\
\hline \multicolumn{5}{|c|}{ Postoperative POP-Q assessment (mean (SD)) } \\
\hline $\mathrm{Aa}$ & $-2.2(1.1)$ & $-2.2(1.1)$ & $-1.8(0.8)$ & 0.724 \\
\hline $\mathrm{Ba}$ & $-3.0(0)$ & $-2.4(0.9)$ & $-2.8(0.4)$ & 0.291 \\
\hline c & $-8.4(0.5)$ & $-8.0(0.7)$ & $-8.6(0.5)$ & 0.330 \\
\hline gh & $3.6(0.5)$ & $3.8(0.4)$ & $4.0(0.7)$ & 0.565 \\
\hline $\mathrm{pb}$ & $3.4(0.5)$ & $3.2(0.4)$ & $3.0(0.7)$ & 0.565 \\
\hline TVL & $10.0(0)$ & $10.0(0.7)$ & $9.4(1.1)$ & 0.418 \\
\hline Ap & $-1.6(2.6)$ & $-2.8(0.4)$ & $-2.8(0.4)$ & 0.638 \\
\hline Bp & $-2.8(0.4)$ & $-2.8(0.4)$ & $-2.8(0.4)$ & 1.000 \\
\hline D & $-8.6(0.9)$ & $-8.6(0.9)$ & N.A. & 1.000 \\
\hline
\end{tabular}

SSHP = vaginal sacrospinous hysteropexy, $\mathrm{LSH}=$ laparoscopic sacrohysteropexy, $\mathrm{VH}=$ vaginal hysterectomy $I Q R=$ interquartile range. $P O P-Q=$ pelvic organ prolapse quantification system, $S D=$ standard deviation

* p value using Chi-square test or Kruskal-Wallis as appropriate

In table 2, the postoperative prolapse complaints (assessed with UDI, DDI and IIQ) and health-related quality of life (SF-36) are showed. After LSH, more women reported bothersome pain during defecation as compared to SSHP and VH. Women of the SSHP group scored significantly higher on the item "embarrassment due to urinary incontinence and/or prolapse and/or defecatory problems" of the $\| Q$. No other significant differences between the groups were found regarding prolapse complaints 6 months after prolapse surgery. Furthermore, health-related quality of life was comparable between all groups.
Table 2. Postoperative functional outcome and quality of life. Values are medians (interquartile range) unless stated otherwise.

\begin{tabular}{|c|c|c|c|c|}
\hline Domains & $\operatorname{SSHP}(n=5)$ & LSH $(n=5)$ & $\mathrm{VH}(\mathrm{n}=5)$ & $\mathrm{p}$-value ${ }^{*}$ \\
\hline \multicolumn{5}{|l|}{ Urogenital distress inventory t: } \\
\hline Overactive bladder & $22(0-44)$ & $0(0-17)$ & $0(0-22)$ & 0.481 \\
\hline Urinary incontinence & $33(0-83)$ & $0(0-25)$ & $17(8-25)$ & 0.449 \\
\hline Obstructive micturition & $0(0-17)$ & $0(0-0)$ & $0(0-33)$ & 0.317 \\
\hline Genital prolapse & $0(0-8)$ & $0(0-8)$ & $0(0-8)$ & 1.000 \\
\hline Pain & $0(0-17)$ & $33(8-50)$ & $0(0-8)$ & 0.066 \\
\hline \multicolumn{5}{|l|}{ Defecatory distress inventory $t$ : } \\
\hline Obstipation & $0(0-25)$ & $0(0-0)$ & $0(0-25)$ & 0.289 \\
\hline Obstructive defecation & $0(0-0)$ & $0(0-4)$ & $0(0-8)$ & 0.311 \\
\hline Pain & $0(0-0)$ & $17(0-25)$ & $0(0-0)$ & 0.031 \\
\hline Incontinence & $0(0-0)$ & $0(0-8)$ & $0(0-0)$ & 0.368 \\
\hline \multicolumn{5}{|l|}{ Incontinence impact questionnaire ¥: } \\
\hline Mobility & $11(0-33)$ & $0(0-22)$ & $0(0-28)$ & 0.758 \\
\hline Physical & $0(0-33)$ & $0(0-0)$ & $0(0-8)$ & 0.265 \\
\hline Social & $0(0-17)$ & $0(0-6)$ & $0(0-0)$ & 0.581 \\
\hline Embarrassment & $17(0-33)$ & $0(0-0)$ & $0(0-0)$ & 0.031 \\
\hline Emotion & $0(0-22)$ & $0(0-0)$ & $0(0-11)$ & 0.311 \\
\hline \multicolumn{5}{|l|}{ Short form-36r: } \\
\hline Physical functioning & $95(83-98)$ & $90(80-93)$ & $85(70-93)$ & 0.392 \\
\hline Social functioning & $100(69-100)$ & $100(94-100)$ & $100(81-100)$ & 0.677 \\
\hline Role limitations physical & $100(63-100)$ & $100(100-100)$ & $100(63-100)$ & 0.362 \\
\hline Role limitations emotional & $100(50-100)$ & $100(100-100)$ & $100(50-100)$ & 0.584 \\
\hline Mental health & $88(62-88)$ & $92(82-92)$ & $84(72-92)$ & 0.560 \\
\hline Vitality & $75(55-90)$ & $80(75-83)$ & $70(33-75)$ & 0.204 \\
\hline Bodily pain & $100(72-100)$ & $90(68-95)$ & $90(56-100)$ & 0.746 \\
\hline General health perception & $85(70-88)$ & $80(48-88)$ & $65(60-83)$ & 0.571 \\
\hline Health change & $100(38-100)$ & $75(50-88)$ & $50(50-100)$ & 0.903 \\
\hline
\end{tabular}

SSHP = vaginal sacrospinous hysteropexy, $L S H=$ laparoscopic sacrohysteropexy, $\mathrm{VH}=$ vaginal hysterectomy t $0=$ no symptoms or not bothersome to $100=$ most bothersome symptoms

$10=$ best quality of life to $100=$ worst quality of life

$0=$ worst quality of life to 100 = best quality of life

Table 3 demonstrates the mean displacement of the anterior vaginal wall, the posterior vaginal wall and the cervix/vaginal vault during straining for each surgical procedure. Furthermore, the angle of displacement of the cervix/vaginal vault during straining is displayed in this table. Pelvic organ mobility was not significantly different between the three groups. In addition, no difference was found in the angle of displacement of the cervix/vaginal vault during straining between the three groups. Figure 4 illustrates the range of the pelvic organ mobility between groups. The largest dispersion was found in the $\mathrm{VH}$ group. 
Table 3. Pelvic organ mobility: displacement per area $(\mathrm{mm})$. Values are medians (interquartile range)

\begin{tabular}{|c|c|c|c|c|}
\hline Characteristics & SSHP ( $n=5)$ & LSH (n=5) & $\mathrm{VH}(\mathrm{n}=5)$ & p-value* \\
\hline \multicolumn{5}{|l|}{ Anterior vaginal wall } \\
\hline $25 \%$ of max straining & $11.1(10.0-21.8)$ & $16.8(15.4-23.7)$ & $22.1(1.3-27.9)$ & 0.566 \\
\hline $50 \%$ of max straining & $21.0(15.6-28.8)$ & $22.6(17.6-29.1)$ & $18.7(8.5-31.0)$ & 0.914 \\
\hline $75 \%$ of max straining & $24.9(16.5-30.7)$ & $23.9(15.9-32.1)$ & $25.5(9.6-34.9)$ & 0.932 \\
\hline $100 \%$ of max straining & $23.9(19.7-33.5)$ & $20.5(16.8-31.6)$ & $20.6(13.5-35.2)$ & 0.677 \\
\hline \multicolumn{5}{|l|}{ Posterior vaginal wall } \\
\hline $25 \%$ of max straining & $14.1(11.8-24.1)$ & $21.1(19.0-25.5)$ & $23.6(2.0-30.8)$ & 0.566 \\
\hline $50 \%$ of max straining & $24.4(18.1-30.2)$ & $27.9(21.7-31.9)$ & $20.5(10.3-34.4)$ & 0.677 \\
\hline $75 \%$ of max straining & $24.7(18.9-35.2)$ & $29.7(20.3-34.5)$ & $26.0(11.8-37.1)$ & 0.878 \\
\hline $100 \%$ of max straining & $22.7(16.4-35.2)$ & $23.6(21.1-33.3)$ & $22.7(16.4-35.2)$ & 0.651 \\
\hline \multicolumn{5}{|l|}{ Cervix/vaginal vault } \\
\hline $25 \%$ of max straining & $10.6(9.5-23.5)$ & $17.1(10.9-21.0)$ & $18.2(1.3-30.7)$ & 0.914 \\
\hline $50 \%$ of max straining & $18.5(13.2-28.7)$ & $21.3(14.2-29.0)$ & $17.1(8.0-34.1)$ & 0.852 \\
\hline $75 \%$ of max straining & $18.4(13.7-28.6)$ & $18.9(13.9-31.8)$ & $21.5(9.0-38.7)$ & 0.932 \\
\hline $100 \%$ of max straining & $17.8(16.1-34.8)$ & $18.2(11.3-34.8)$ & $19.5(11.2-38.4)$ & 0.961 \\
\hline \multicolumn{5}{|c|}{ Cervix/vaginal vault: angle of displacement (degrees) } \\
\hline $25 \%$ of max straining & $29.5(22.1-36.6)$ & $32.8(12.5-47.2)$ & $16.0(-26.3-45.1)$ & 0.698 \\
\hline $50 \%$ of max straining & $25.3(22.0-28.2)$ & $25.7(8.7-44.9)$ & $33.1(12.2-38.9)$ & 0.878 \\
\hline $75 \%$ of max straining & $26.2(20.1-30.2)$ & $29.2(7.8-47.6)$ & $31.7(20.1-37.8)$ & 0.651 \\
\hline $100 \%$ of max straining & $23.7(18.8-28.5)$ & $30.6(11.3-53.8)$ & $31.1(17.7-36.3)$ & 0.403 \\
\hline \multicolumn{5}{|l|}{ Vaginal axes (degrees) } \\
\hline Upper vaginal axist & $36.3(26.0-50.7)$ & $43.3(35.2-60.9)$ & $13.7(11.6-29.9)$ & 0.042 \\
\hline Middle vaginal axis $\dagger$ & $102.8(85.8-123.9)$ & $116.5(98.8-129.9)$ & $98.6(87.6-102.7)$ & 0.237 \\
\hline Lower vaginal axist & $116.4(109-124.9)$ & $106.8(97.7-113.1)$ & $108.3(105.8-116.9)$ & 0.151 \\
\hline Upper-middle vaginal axis & $120.7(97-126.4)$ & 110 (89.5-134.2) & $115.4(89.6-126.3)$ & 0.878 \\
\hline Middle-lower vaginal axis & $165.7(152.8-183.4)$ & $197.9(173.3-207.8)$ & $153.7(146.7-173)$ & 0.057 \\
\hline
\end{tabular}

After $\mathrm{VH}$, the vaginal axis of the upper vaginal region was significantly inferior as compared to SSHP and LSH (median 13.7 degrees, compared to resp. 36.3 and 43.3, p 0.042 , table 3). After $\mathrm{VH}$, the upper part of the vagina seems to be more posterior as compared to LSH and SSHP (figure 5). Regarding middle and lower vaginal region, no difference between procedures was found in vaginal axes.
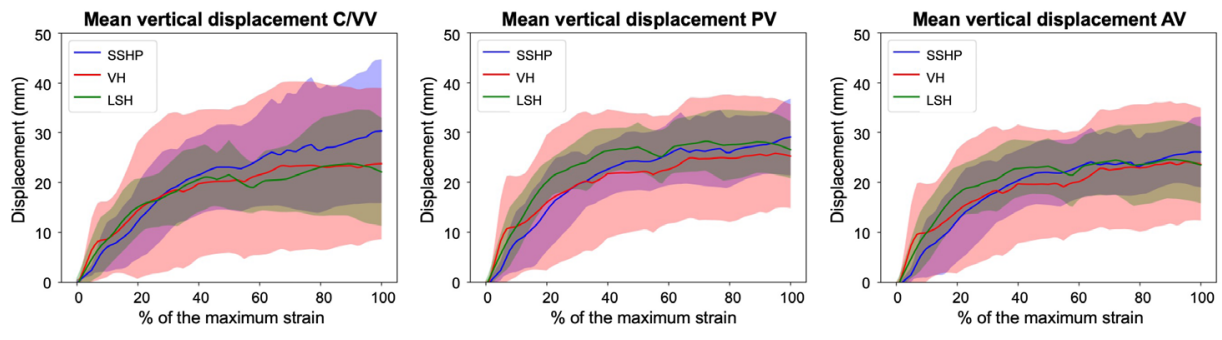

FIGURE 4. Mean vertical displacement ( $\mathrm{mm}$ ) in anterior vaginal wall (AV), posterior vaginal wall (PV) and cervix/vaginal vault (C/NV) assessed with MRI 6 months after vaginal sacrospinous hysteropexy (SSHP), vaginal hysterectomy (VH) and laparoscopic sacrohysteropexy (LSH).

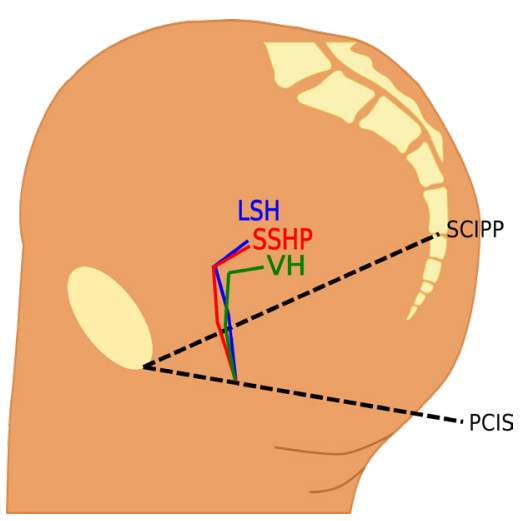

FIGURE 5. Vaginal axes after POP surgery. $L S H=$ laparoscopic sacrohysteropexy, SSHP $=$ sacrospinous hysteropexy, $\mathrm{VH}=$ vaginal hysterectomy, $\mathrm{SCIPP}=$ sacro-coccygeal inferior pubic point, $\mathrm{PCIS}=$ pelvic inclination correction system

\section{DISCUSSION}

In the present study, dynamic MRI 6 months after SSHP demonstrated no difference in pelvic organ mobility of the anterior vaginal wall, neither in the posterior vaginal wall and cervix/vaginal vault as compared to LSH and VH. Furthermore, the angle of displacement of the cervix/vaginal vault was comparable between the three groups and no significant difference was found in the vaginal axes after SSHP as compared to $\mathrm{LSH}$ and $\mathrm{VH}$. Based on these data, no explanation for the higher recurrence rate of cystocele after SSHP was found. 
Little is known about pelvic organ mobility after prolapse surgery. To the best of our knowledge, this is the first study to compare pelvic organ mobility after hysteropexy and hysterectomy. One prospective clinical trial compared preoperative pelvic organ mobility to postoperative pelvic organ mobility in women who underwent anterior and/or posterior mesh-repair surgery [27]. In this study, pelvic organ mobility improved significantly after surgery.

Vaginal configuration after POP surgery has been described in several studies. After attaching the post-hysterectomy vaginal vault to the sacrospinous ligaments, static magnetic resonance images demonstrate a distortion of the vaginal anatomy by pulling the upper vaginal plane superiorly and at times anteriorly $[24,28]$. This finding is contrary to the common belief that the vagina is pulled posteriorly by a sacrospinous fixation. A possible explanation may be that the vaginal vault is normally situated below the level of the ischial spines and sacrospinous ligaments, but suspension to these ligaments would pull the vagina superiorly resulting in a "straining" effect in the sagittal plane [28]. These findings are mainly in line with our study results. After SSHP, we found the vagina to be pulled more superior as compared to $\mathrm{VH}$, but vaginal axis after SSHP was comparable to LSH. A retrospective cohort study assessed vaginal axes using dynamic MRI in women after hysterectomy as compared to women with a uterus in place [25]. After hysterectomy, the middle vaginal axis was positioned more anteriorly as compared to the middle vaginal axis with a uterus in place. Furthermore, the angles between upper-middle vagina and between middle-lower vagina were more obtuse in women after hysterectomy, suggesting straightening of the vaginal axis. These findings are not in line with our study. After $\mathrm{VH}$, we found a more posterior position of the upper vagina as compared to SSHP and LSH, with no differences of the middle and lower vaginal region. A possible explanation for this difference might be in the fact that in the latter study hysterectomy was performed for various conditions, whereas in our study only women after POP treatment were included.

This study has several strengths and limitations. The strength of this report is that this is the first study to compare anatomical and functional results of SSHP, LSH and VH by MRI measurement including dynamic evaluation. Furthermore, MR images were analyzed by one experienced researcher, with no risk of inter-observer variability. In addition, images were obtained by a standardized protocol.

A limitation of this study is the small sample size. In this pilot study only 15 women were included in total (5 of each group). Furthermore, we did not investigate recurrences of POP in this study. It is unclear whether or not angle of displacement and vaginal axes are different in these women compared to our study group. All measurements were done in the midsagittal plane. The vaginal axis, especially in case of SSHP, might point to the right side what is not reflected by midsagittal measurements. Lacking of the 3D-aspect of the vaginal axis could be considered a weakness of this study. To evaluate pelvic organ mobility, patients were instructed to put strain of their pelvic floor by performing a Valsalva maneuver. However, the effect of Valsalva on the extent of POP (and thus pelvic organ mobility) is dependent on the instructions by the physician and the knowledge and the ability of the patients to relax (strain) their pelvic floor muscles [29]. In addition, repeating the Valsalva maneuver increases the induced maximal strain. Interpreting pelvic organ mobility using this method could be subjective. Literature suggests that upright MRI scanning both at rest and maximal straining shows a larger extent of the prolapse than that observed during supine straining [30]. At time of this study, an upright MRI scanner was not available in our clinic. However, it would be interesting to assess pelvic organ mobility using an upright MRI scanner.

In conclusion, we found no difference in pelvic organ mobility after SSHP as compared to other POP operations. Based on these data, we cannot explain the higher recurrence risk in the anterior compartment after SSHP. This might be due to the small sample size of this pilot study. It will be interesting to evaluate pelvic organ mobility after SSHP in a larger sample size and in women who have recurrence or de novo cystocele to further assess the relation between pelvic organ mobility and the occurrence of anterior vaginal wall prolapse. Furthermore, assessing pelvic organ mobility using an upright MRI scanner could provide additional insights. 


\section{REFERENCES}

1. Jha S, Cutner A, Moran P. The UK national prolapse survey: 10 years on. Int Urogynecol J 2018:29:795-801

2. Van IJsselmuiden MN, Detollenaere RJ, Gerritse MBE, Kluivers KB, Bongers MY, van Eijndhoven HWF. Dutch women's attitudes towards hysterectomy and uterus preservation in surgical treatment of pelvic organ prolapse. Eur J Obstet Gynecol Reprod Biol 2018;220:79-83

3. Korbly NB, Kassis NC, Good MM, Richardson ML, Book NM, Yip S, Saguan D, Gross C, Evans J, Lopes VV, Harvie HS, Sung VW. Patient preferences for uterine preservation and hysterectomy in women with pelvic organ prolapse. Am J Obstet Gynecol 2013:209(5):470

4. Madsen AM, Raker C, Sung VW. Trends in hysteropexy and apical support for uterovaginal prolapse in the united states from 2002 to 2012 Female Pelvic Med Reconstr Surg 2017;23(6):365371

5. Detollenaere RJ, den Boon J, Kluivers KB, Vierhout $M E$, van Eijndhoven HW. Surgical management of pelvic organ prolapse and uterine descent in the Netherlands. Int Urogynecol J 2013;24(5):781-8

6. Schulten SFM, Detollenaere RJ, Stekelenburg J,IntHout J, Kluivers KB, van Eijndhoven HWF Sacrospinous hysteropexy versus vaginal hysterectomy with uterosacral ligament suspension in women with uterine prolapse stage 2 or higher: observational follow-up of a multicentre randomized controlled trial. BMJ 2019;366:15149

7. Dietz V, van der Vaart $\mathrm{CH}$, van der Graaf $\mathrm{Y}$, Heintz P, Schraffordt Koops SE. One-year follow-up after sacrospinous hysteropexy and vaginal hysterectomy for uterine descent: a randomized study. Int Urogynecol J 2010;21(2):209-16

8. Petri E, Ashok K. Sacrospinous vaginal fixation - current status. Acta Obstet Gynecol Scand 2011;90:429-36

9. Morgan DM, Rogers MA, Huebner M, Wei JT, Delancey JO. Heterogeneity in anatomic outcome of sacrospinous ligament fixation for prolapse: a systematic review. Obstet Gynecol 2007:109:1424-33.

10. Aigmueller T, Riss P, Dungl A, Bauer H. Long-term follow-up after vaginal sacrospinous fixation: patient satisfaction, anatomical results and quality of life. Urogynecol J Pelvic Floor Dysfunct 2008:19:965-9

11. DeLancey JO, Morgan DM, Fenner DE, Kearney R, Guire K, Miller JM, Hussain H, Umek W, Hsu $Y$, Ashton-Miller JA. Comparison of levator ani muscle defects and function in women with and without pelvic organ prolapse. Obstet Gynecol 2007;109:295-302

12. Kobi M, Flusberg M, Paroder V, Chernyak V. Practical guide to dynamic pelvic floor MRI. J Magn Reson Imaging 2018;47:1155-70

13. Rubod C, Lecomte-Grosbas P, Brieu M, Giraudet G, Betrouni N, Cosson M. 3D simulation of pelvic system numerical simulation for a better understanding of the contribution of the uterine ligaments. Int Urogynecol J 2013;24:2093

14. Chen L, Ashton-Miller JA, DeLancey JO. A 3D finite element model of anterior vaginal wall support to evaluate mechanisms underlying cystocele formation. J Biomech 2009:42(10):1371-7.

15. Jeanditgautier $\mathrm{E}$, Mayeur $\mathrm{O}$, Brieu M, Lamblin $\mathrm{G}$. Rubod C, Cosson M. Mobility and stress analysis of different surgical simulations during a sacral colpopexy, using a finite element model of the pelvic system. Int Urogynecol J 2016;27(6):951-7

16. Bump RC, Mattiasson A, Bo $k$ et al. The standardization of terminology of female pelvic organ prolapse and pelvic floor dysfunction. Am J Obstet Gynecol 1996;175:10-7.

17. Ware JE, Kosinksi M, Keller SD. SF-36 physical and mental component summary measures - a user's manual. 1994. New England Medical Center, The Health Institute.

18. van der Vaart $\mathrm{CH}$, de Leeuw JR, Roovers JP Heintz AP. Measuring health-related quality of life in women with urogenital dysfunction: the urogenital distress inventory and incontinence impact questionnaire revisited Neurourol Urodyn 2003;22:97-104.

19. Roovers JP, van der Bom JG, van der Vaart $\mathrm{CH}$ Heintz AP. Prediction of findings at defecography om patients with genital prolapse. Br J Obstet Gynaecol 2005;112:1547-53

20. Lamers LM, Stalmeier PF, McDonnell K, Krabbe PF, van Busschbach JJ. Measuring the quality of life economic evaluations: the Dutch EQ-5D tariff. Ned Tijdschr Geneeskd 2005;149:1574-8

21. Schweitzer KJ, de Jong M, Milani AL. Prolaps en seks: hoe meten we de relatie. Ned Tijdschr Obs Gyn 2008;121:79-82

22. Klein S, Staring M, Murphy K, Viergever MA, Pluim JP. Elastix: a toolbox for intensity-based medical image registration. IEEE Trans Med Imaging 2010;29(1):196-205

23. Lecomte-Grosbras P, Witz JF, Faye N, Cosson M, Rubod C. Quantification of pelvic mobility on dynamic magnetic resonance images: using mechanical insight to help diagnose pelvic pathologies. Strain 2015:51:301-10

24. Sze EH, Meranus J, Kohli N, Miklos JR, Karram MM. Vaginal configuration on MRI after abdominal sacrocolpopexy and sacrospinous ligament suspension. Int Urogynecol J Pelvic Floor Dysfunct 2001;12:375-9

25. Lee DD, Siegelman ES, Chua WY, Arya LA, Harvie HS. Comparison of vaginal axis in women who have undergone hysterectomy versus women with an intact uterus. Female Pelvic Med Reconstr Surg 2019;25(4):313-17

26. Betschart C, Chen L, Ashton-Miller JA, Delancey JO. On pelvic reference lines and the $M R$ evaluation of genital prolapse: a proposal for standardization using the Pelvic Inclination Correction System. Int Urogynecol J 2013;24(9):1421-1428

27. Alt CD, Brocker KA, Lenz F, Sohn C, Kauczor HU, Hallscheidt P. MRI findings before and after prolapse surgery. Acta Radiol 2014;55(4):495-504

28. Rane A, Lim YN, Withey G, Muller R. Magnetic resonance imaging findings following three different vaginal vault prolapse repair procedures: a randomized study. Aust N Z J Obstet Gynaecol 2004:44:135-9.

29. Tumbarello JA, Hsu Y, Lewicky-Gaupp C, Rohrer S. DeLancey JO. Do repetitive Valsalva maneuvers change maximum prolapse on dynamic MRI? Int Urogynecol J 2010;21(10):1247-51

30. Grob ATM, Olde Heuvel J, Futterer JJ, Massop D, Veenstra van Nieuwenhoven AL, Simonis FFJ, van der Vaart $\mathrm{CH}$. Underestimation of pelvic organ prolapse in the supine straining position based on magnetic resonance imaging findings. Int Urogynecol J 2019;30(11):1939-44 


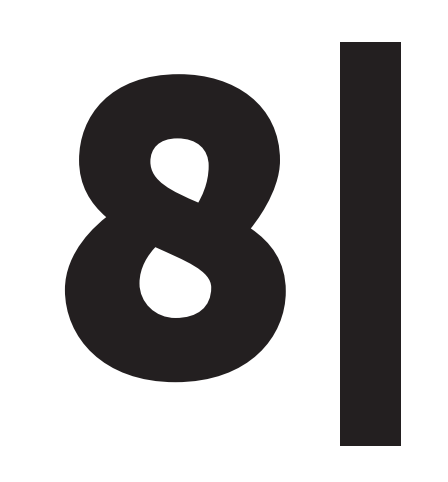

GENERAL DISCUSSION AND FUTURE PERSPECTIVES 
This thesis focuses on the surgical management of uterine prolapse with emphasis on surgical procedures for hysteropexy. We aimed to answer the following questions: do women prefer uterus preservation or hysterectomy in case of uterine prolapse? How do Dutch gynaecologists treat pelvic organ prolapse (POP) and which type of hysteropexy should be done in case of uterine descent?

\section{HOW DO DUTCH GYNAECOLOGISTS TREAT POP?}

The choice for surgical treatment for POP mainly depends on the prolapse severity, symptoms, the general health of the woman, and the preference and capabilities of the surgeon [1]. Practice pattern variation (PPV) is defined as the difference in care that cannot be explained by the underlying medical condition. We calculated PPV for POP and urinary incontinence (UI) in the Netherlands using data of health declaration codes (CHAPTER 2). In our small country, we found a high PPV in surgical management of POP and UI with respect to choose for surgical treatment and the type of surgery. Surgical treatment regarding uterine descent varies per hospital: non-teaching hospitals performed significantly more hysterectomies in relation to uterus-preserving techniques compared with teaching and university hospitals. Furthermore, the ratio between conservative and surgical management of POP and UI varies per hospital. As a consequence, a patient's odds of undergoing surgery for POP or UI seems to depend more on where the patient lives and to which hospital she is referred than on clinical circumstances.

In addition to practice pattern variation on POP and $\mathrm{UI}$ in general, we have studied practice variation on laparoscopic sacrohysteropexy and sacrocolpopexy in specific. In the Netherlands, a standardized approach or published guideline for these procedures is lacking. We performed a nationwide survey to assess variation among Dutch gynaecologist in executing laparoscopic sacrohysteropexy and laparoscopic sacrocolpopexy (CHAPTER 3). In the Netherlands these laparoscopic operations are performed only by a selected group of gynaecologists. Nevertheless, we found great variations in operative technique for these procedures. Variation was found in several important aspects of the surgery, such as level of dissection along the anterior and posterior vaginal wall, the type of mesh used, the type of sutures used, the tension of the implanted mesh, and reperitonealisation of the mesh.

\section{DISCUSSION ON PRACTICE PATTERN VARIATION OF POP}

Regional variation in the use of surgical procedures has long been recognized. In
1938, in some school districts in the UK more than half of the children had undergone tonsillectomies by age of 18 , as compared with fewer than $10 \%$ in other districts, even though only 7 miles separated [2]. In the last decades, several studies have documented wide regional variation in the rates of numerous other surgical procedures, e.g. surgery for hip, knee and spine, coronary bypass surgery and prostatectomy [4,5]. Furthermore, geographical variation in hysterectomy rates and surgical routes for hysterectomy (vaginal/abdominal) for benign diseases was observed in The Netherlands [5].

Little is known on practice pattern variation of POP. In the United States, a variation in the current practice of colpocleisis was found, likely because of lack of long-term prospective studies [6]. Furthermore, a variable surgical policy in case of uterine descent was found in the Netherlands and the United Kingdom [7,8]

Several factors contributing to PPV have been described. First, variation in patient characteristics and demand for surgery might contribute to PPV [4]. Some regions might have a higher rate of surgical procedures because of greater demand for surgery. Second, the preference of the patient can contribute to PPV [4]. For some clinical conditions, the decision to intervene should depend as much on patient preferences as on scientific evidence. Third, scientific uncertainty contributes to PPV [3]. In the evidence of professional consensus and evidence-based guidelines, individual or small groups of physicians can hold on their own clinical rules of defining which patient needs surgery. This might result in the "surgical signature" of a region: rates for specific surgical procedures that are peculiar to a region sometimes differ substantially between surrounding regions [3,9]. Last, surgical innovations and new technologies can pronounce PPV by increasing the number of therapeutic alternatives available to clinicians [4].

PPV is considered to be a great problem in controlling medical costs. Furthermore, PPV in the ratio between conservative and surgical management of POP might imply over- and undertreatment. In case of overtreatment, the patient is exposed to the unnecessary risks of surgery. However, in case of undertreatment, the patient might not receive adequate treatment. Since a gold standard for treating POP is still a matter of debate, the main question arises what the optimal ratio is between conservative and surgical management. As a result, it is difficult to conclude whether or not we observed over- or undertreatment.

To reduce PPV in POP, studies with respect to conservative versus surgical treatment, and randomized controlled trials between different types of surgery are needed to 
establish evidence-based guidelines to prevent over- and undertreatment in the future. Furthermore, shared decision making (SDM) might be an important tool in reducing PPV. Differences in the extent of which doctors incorporate preferences of the individual patient into treatment decisions might be an important factor underlying regional variation in surgery rates [4]. The concept of SDM is particularly relevant for preference-sensitive decisions, when two or more options are medically appropriate and the best choice for the individual patient depends on their own assessment of relative importance of different characteristics and outcomes [10]. Therefore, SDM can be perfectly applied in POP.

Patient decision aids are frequently used to support SDM. These tools provide patients with detailed information of the risks and benefits of different treatments for specific conditions. It can be delivered across various media formats, including mobile device applications, printed leaflets and interactive computer programs [11]. In the Netherlands, to our knowledge, no decision aid regarding treatment of POP has been developed so far. No studies have assessed whether decision aids reduce variation in procedure rates but several trials have shown significant decreases in overall surgery rates following their implementation [12-14]. Randomised controlled trials like the LAVA trial could not only help to establish evidence-based guidelines, but also help to develop a decision tool for SDM to reduce PPV.

\section{DISCUSSION ON VARIATION OF SURGICAL TECHNIQUE}

Several studies have demonstrated variation among surgeons in surgical technique of POP procedures. Considerable variation was found among UK-based surgeons in both native and mesh-augmented POP repairs [15]. In the Netherlands a variation has been found among Dutch gynaecologists in performing anterior colporrhaphy [16]. Furthermore, an international survey among members of the International Urogynaecology Association (IUGA) demonstrated variation in anterior and posterior vaginal wall repair [17]

The great variations in surgical technique may be a consequence of the lack of evidence as to what the best practice is. Furthermore, different techniques could affect surgical outcomes. To monitor the outcome and long-term complications of (laparoscopic) POP repair, consensus on how to perform these procedures seems mandatory. It may not be possible to achieve complete standardization of the surgical procedures, as some points depend on the preference of the gynaecologists. In addition, it can be discussed whether or not complete standardization would be relevant, as some variables probably do not contribute to the surgical outcome (e.g. trocar placement, cystoscopy afterwards). However, in some keypoints of the procedure, standardization could be realizable and beneficial.

Regarding laparoscopic sacrohysteropexy and laparoscopic sacrocolpopexy, in our opinion these keypoints are:

\section{Extent of dissection}

Attachment of the mesh to the cervix or vaginal vault (anterior and/or posterior)

Type of sutures to be used for attachment of the mesh

It is unknown to what level these keypoints contribute to the surgical outcome. Furthermore, conducting a guideline is complex as there is no evidence in the specific parts of the procedure. First, well-designed prospective studies or randomized controlled trials with regard to the specific parts of laparoscopic sacrohysteropexy and sacrocolpopexy are needed to provide evidence for the best surgical technique.

\section{WHAT DO WOMEN WANT?}

We studied Dutch women's attitudes and preferences regarding surgical management of uterine prolapse in a cross-sectional study using a questionnaire (CHAPTER 4). In case functional and anatomical outcomes after hysterectomy and uterus preserving surgery were equal, more women expressed preference for uterus preservation (43\%) over hysterectomy $(27 \%)$. The majority of women expected a similar improvement in sexuality and body image after both treatment modalities. Most common reasons to choose for hysterectomy were menstrual disorders, the believe that the uterus has become unnecessary and to avoid future problems. Reasons for uterus preservation were a less invasive procedure, the wish to stay as complete as possible and not removing a healthy organ. Treatment success, risk for urinary incontinence after surgery and complication risk were the most important factors when choosing a type of surgical intervention. The future risk of endometrial cancer was for the majority of women not a reason to choose for hysterectomy

\section{DISCUSSION ON PATIENT PREFERENCES}

Our study results are in line with two preference studies conducted in the USA, Germany and Russia [18-20].

Several studies determined patients' expectations regarding POP surgery. Physical improvement and symptom release are thought to be the most important factor to 
consider surgery a success, along with restoration of "normality" [21,22]. The majority of patients that are scheduled for POP surgery fear de novo symptoms, particularly urinary incontinence [22]. In our study, we have stated that hysterectomy might be more often associated with urinary incontinence. Unfortunately, no counter-factual statements were used providing advantages of hysterectomy over uterine preservation.

It is generally accepted that the uterus plays a passive role in the development of POP [23]. As a result, hysterectomy may not be necessary, to correct the underlying defect in the apical vaginal support structures. Incorporating patient preferences and expectations of POP surgery when choosing a surgical intervention is of importance to choose the best surgical option for the specific patient. Since the majority of women prefer uterine preservation, uterine sparing surgical options should be assessed and considered in the surgical decision-making process.

\section{HOW SHOULD UTERINE PROLAPSE BE TREATED?}

Traditionally, uterine prolapse was treated by vaginal hysterectomy and suspension of the vaginal vault. However, the uterus is not the cause but only a passive structure in the development of prolapse [23] and more women express a preference for uterus preservation [18-20,24]. Several studies have demonstrated that uterine suspension is as effective and safe as prolapse surgery including vaginal hysterectomy $[25,26]$. To suspend the uterus laparoscopic, robotic, abdominal and vaginal procedures have been described. To date, no randomized controlled trials are available to compare these different routes. As a result, whether or not these operations have comparable anatomical and functional outcomes remains unclear.

Vaginal sacrospinous hysteropexy is the most studied technique for uterus suspension, its efficacy has been demonstrated in several RCT'S [27-29]. Although, laparoscopic sacrohysteropexy seems to become increasingly popular [8,30] laparoscopic sacrohysteropexy has not been compared directly in a randomized trial against vaginal sacrospinous hysteropexy. Therefore, we initiated the LAVA-trial (LAparoscopic sacrohysteropexy versus VAginal sacrospinous hysteropexy in treatment of uterine prolapse) in 2013 (CHAPTER 5 AND 6). Women with uterine prolapse stage two or higher without previous prolapse surgery were randomized between laparoscopic sacrohysteropexy and vaginal sacrospinous hysteropexy. Main findings were that after a follow-up of 12 months, laparoscopic sacrohysteropexy was non-inferior to vaginal sacrospinous hysteropexy regarding anatomical recurrence in the apical compartment with bothersome symptoms or repeat surgery or pessary use compared to vaginal sacrospinous hysteropexy. Furthermore, quality of life did not differ between the surgeries. However, more bothersome symptoms of overactive bladder and faecal incontinence were reported after laparoscopic sacrohysteropexy. Dyspareunia was more frequently reported after vaginal sacrospinous hysteropexy.

\section{DISCUSSION ON THE LAVA TRIAL}

The LAVA trial is the first randomized controlled trial comparing two techniques for hysteropexy. The study demonstrated non-inferiority to vaginal sacrospinous hysteropexy for surgical failure in the apical compartment. Furthermore, no differences were found in anatomical and surgical failure in other compartments, neither in sexual functioning and quality of life. Our findings are mainly in line with data of several studies of vaginal sacrospinous hysteropexy versus vaginal hysterectomy and laparoscopic sacrohysteropexy versus vaginal hysterectomy

When looking at the vaginal sacrospinous hysteropexy, we observed a higher overall surgical failure after this procedure at one-year follow-up as compared to the SAVE U trial $(62.3 \%$ versus $51 \%)$ [27]. This might be caused by a higher incidence of prolapse of the anterior compartment in our study group (56.9\% versus $47 \%$ ), whereas the rates of concomitant anterior colporrhaphy were comparable (98.4\% and $97 \%)$. This finding may be attributed to the large variation in surgical technique of anterior colporrhaphy, which might lead to different surgical outcomes [16]. Compared to the randomized controlled trial of Dietz et al. we found less anatomical recurrences of the apical compartment (3.4\% versus $21 \%$ ) after vaginal sacrospinous hysteropexy [29]. In the randomized controlled trial of Dietz et al, which was performed between 2004 and 2006, vaginal sacrospinous hysteropexy was perhaps a relatively novel technique. The differences between anatomical recurrence might be explained by more experience nowadays and improvement in surgical skills.

Regarding laparoscopic sacrohysteropexy, our findings are mainly in line with other prospective studies and RCTs. In a prospective study by Price et al, one surgical failure (2\%) of the apical compartment was observed at 10-week follow-up, compared to $1.9 \%$ in our study [31]. In a prospective observational study 3 women (2\%) required reoperation for apical support for symptomatic prolapse at a mean follow-up of 2.1 years [32]. In our study, no reoperation of the apical compartment was observed at a follow-up of 12 months, probably due to the shorter follow-up. Furthermore, our findings are mainly in line with a randomised study comparing laparoscopic sacrohysteropexy to vaginal hysterectomy [33]. However, we found lower reoperation 
rates of the apical compartment at one-year follow-up (0\% versus $6 \%$ ). Differences in sample size (37 versus 59) and subtle differences in surgical protocol might explain this finding

Although the LAVA trial demonstrated no differences in primary outcome, we found some differences in secondary outcomes. First, more bothersome symptoms of overactive bladder were reported after laparoscopic hysteropexy. This finding seems mostly due to persistence of preoperative overactive bladder symptoms. Therefore, it implicates that women with preoperative symptoms of overactive bladder benefit more from vaginal sacrospinous hysteropexy. Second, more bothersome faecal incontinence has been reported after laparoscopic sacrohysteropexy as compared to vaginal sacrospinous hysteropexy. Since treatment options are limited and associated with side-effects, faecal incontinence is thought to be a devastating outcome [34,35]. Third, dyspareunia was reported almost three times as often after vaginal sacrospinous hysteropexy as compared to laparoscopic sacrohysteropexy. These differences in secondary outcomes may help in the process of shared decision making and choosing the optimal surgical route for the individual patient. One could hypothesize that for example a woman with overactive bladder symptoms is better off with a vaginal sacrospinous hysteropexy and sexually active women with a laparoscopic sacrohysteropexy.

The outcomes of the LAVA trial were evaluated at 12 months' follow-up. This follow-up is short for a treatment that should last a lifetime and recurrences may further develop during longer follow-up. As stated in our study protocol, included women will be followed until 60 months after surgery. However, a recent cohort study demonstrated that the highest risk for undergoing repeat surgery for POP is within the first year [36].

A major problem in evaluating results after POP surgery is the significant variation in how outcomes are reported [37]. It is known that treatment success varies widely depending on the definition of surgical success. However, definitions of treatment success that require patient reported outcome postoperatively are more clinically relevant and meaningful to the patient than those that include anatomical criteria only [37]. The absence of vaginal bulge symptoms postoperatively has a significant relationship with patient's assessment of overall improvement, while anatomical success has not. In the LAVA trial, we have chosen a composite outcome measure including anatomical outcome of the apical compartment, absence of bothersome bulge complaints and repeat surgery or pessary use. Furthermore, we reported on outcomes of the anterior and posterior compartment and also reported on outcomes using the hymen as threshold for success.

\section{PELVIC ORGAN MOBILITY AND VAGINAL AXES AFTER POP SURGERY}

The risk of recurrent prolapse of the anterior vaginal wall after vaginal sacrospinous hysteropexy is often discussed with incidences ranging from $5.8-21.3 \%$ [38,39]. We hypothesized that pelvic organ mobility, which is defined as the displacement of pelvic organs between rest and maximal straining, after vaginal sacrospinous hysteropexy is increased, which might contribute to the high occurrence of postoperative cystocele after this surgery. Pelvic organ mobility and the vaginal axes after vaginal sacrospinous hysteropexy were assessed by dynamic magnetic resonance imaging and compared with vaginal hysterectomy with uterosacral vault suspension and laparoscopic sacrohysteropexy (CHAPTER 7). We found no differences in pelvic organ mobility 6 months after vaginal sacrospinous hysteropexy as compared to vaginal hysterectomy and laparoscopic sacrohysteropexy. After vaginal hysterectomy, a more posterior position of the upper vagina was found as compared to vaginal sacrospinous hysteropexy and laparoscopic sacrohysteropexy. Based on our data, the higher recurrence risk in the anterior compartment after vaginal sacrospinous hysteropexy cannot be explained. This might be due to the small sample size of this pilot study. Little is known about pelvic organ mobility after prolapse surgery. To the best of our knowledge, this is the first study to compare pelvic organ mobility after hysteropexy and hysterectomy

It will be interesting to evaluate pelvic organ mobility after vaginal sacrospinous hysteropexy in a larger sample size and in women who have recurrence or de novo cystocele to further assess the relation between pelvic organ mobility and the occurrence of anterior vaginal wall prolapse. Furthermore, assessing pelvic organ mobility using an upright MRI scanner could provide additional insights.

\section{THE ROLE OF LAPAROSCOPIC UTERINE PRESERVATION}

Surgery for POP is performed traditionally via abdominal or vaginal approaches. Advances in minimal invasive surgery have led to an increase in adoption of laparoscopic techniques [40]. The laparoscopic approach has been successfully adopted for many procedures previously relied on an abdominal route. Laparoscopy should be considered only as a mode of abdominal access and not a change in operative technique [41]. The possible advantages of laparoscopic POP repair include improved visualization of anatomy because of laparoscopic magnification, insufflation effects and improved haemostasis; shortened hospitalization resulting in potential costs reduction; decreased postoperative pain and more rapid recovery and return to work; and better cosmetic appearance of smaller incisions [42] 
With the advantages of laparoscopic POP repair, laparoscopic procedures have mostly replaced the open abdominal procedures [43]. The main question is when to treat POP vaginally or laparoscopically. Hysteropexy is a relatively novel technique for treating uterus prolapse. Traditionally, surgical repair of uterine descent involved vaginal vault suspension utilizing native tissue or mesh with concurrent hysterectomy. Nowadays, there is a growing interest in uterine conservation and hysteropexy procedures. Although these procedures were first described more than a century ago, they have gained interest over the past decade [25]. Laparoscopic hysteropexy has never been compared to vaginal hysteropexy in a randomized controlled trial. However, abdominal sacrocolpopexy of the vaginal vault is well described in the literature. When compared with sacrospinous fixation of the vaginal vault, abdominal sacrocolpopexy has been shown to have a lower rate of recurrent apical prolapse, less post-operative dyspareunia and a lower reoperation rate. However, the open abdominal procedure takes longer to perform, has a longer recovery time and is more expensive [1]. Randomized controlled trials comparing laparoscopic to open abdominal sacrocolpopexy demonstrated less blood loss and shorter hospital stay after the laparoscopic approach, with equivalent functional and anatomical outcomes [43,44]

When looking at the evidence of laparoscopic sacrocolpopexy, laparoscopic hysteropexy in case of uterine descent seems promising. However, randomized controlled trials are needed to compare the efficacy of various hysteropexy procedures and to develop a decision aid with risks and benefits of both the vaginal and laparoscopic surgical route. The LAVA trial is the first randomized controlled trial comparing laparoscopic to vaginal hysteropexy demonstrating non-inferiority of the primary outcome (surgical failure)

Complex operative laparoscopy is associated with a steep and lengthy learning curve. A linear decrease in operation time was found after the initial procedure. In the first 30 consecutive cases, operation time declined rapidly reaching a steady state after 90 cases [45]. When attending a skills lab dedicated to endoscopic suturing and knot tying, stable operation time was achieved after 30 cases. However, when looking at complications and outcomes of the learning curve, adequate learning (no need for conversion to laparotomy, absence of anatomical failure and major complications) was showed after 60 cases [45]. Taking the learning curve into account, and assuming the gynaecologists needs to perform this procedure at a regular base to maintain the experience, it may not be possible to perform laparoscopic POP surgery in each hospital in the Netherlands. Therefore, in our opinion, complex laparoscopic POP surgery nowadays should be limited to specialized urogynaecology centres.

\section{FUTURE PERSPECTIVES}

With respect to future research, we would like to discuss the following topics:

1. Outcomes after POP treatment

2. Conservative versus surgical management of POP

3. Minimally invasive POP procedures

\section{OUTCOMES AFTER POP TREATMENT}

One major problem in evaluating effects of POP surgery is the range of outcome measures, which are not reported in a consistent manner. Treatment success varied widely depending on the definition used [37]. In a recent systematic review on reported outcomes in randomized controlled trials on apical prolapse surgery, 76 outcomes and 66 outcome measures were identified [46]. As a result of heterogenous outcomes, meaningful meta-analysis of POP outcome data is impossible [47]. This problem is not unique for uterine prolapse, it has been observed in surgery for posterior vaginal prolapse, stress urinary incontinence, childbirth perineal trauma research and heavy menstrual bleeding [48,51]. The development of a core-outcome set to standardize reporting outcomes will enable high-quality meta-analyses to be performed in the future. The core-outcomes can be determined by a group of selected stakeholders (for instance health care professionals, researchers and patient representatives. Core outcome have already established in fetal growth restriction and a protocol for developing and implementing core outcomes have been published for endometrioses and stress urinary incontinence [52-54]. In 2012, the International Urogynaecology Association (IUGA) and International Continence Society (ICS) published a terminology report with definitions of surgery and a structure for reporting the outcomes of surgical procedures [55]. However, this document does not define surgical success and failure. An ideal outcome measure should be valid, reliable, responsive, easily assessed, easily interpreted and clinically relevant [37]. Regarding POP surgery, any definition of success should include the absence of vaginal bulge symptoms in addition to anatomical criteria and the absence of re-treatment [37]. In addition, using the hymen as a threshold for anatomical success seems a reasonable and defensible approach since $40 \%$ of parous women have anatomical prolapse POP-Q grade 2 or higher.

However, patient reported outcome measurements (PROM's) on quality of life, functional outcome and sexual functioning are perhaps be the most important outcome since severity of the prolapse does not correlate with prolapse symptoms or the impact on quality of life [56]. In addition, the goals of POP treatment should always 
be reducing the symptoms and improving quality of life. Therefore, these PROM's should always take into account when evaluating surgical outcomes.

\section{CONSERVATIVE VERSUS SURGICAL MANAGEMENT OF POP}

In our study on PPV in the Netherlands, we observed large differences in the ratio between surgical and conservative treatment of POP. The absence of clearly defined guidelines regarding indications for conservative and surgical management of POP might contribute to this finding. Randomized controlled trials comparing conservative versus surgical treatment are lacking but are needed to establish guidelines. In 2015, the PEOPLE study, a prospective randomized controlled clinical trial was started in the Netherlands with the aim to determine non-inferiority of the endpoint (patient improvement) between pessary therapy and surgical treatment of POP.

We analysed practice pattern variation in the Netherlands based on data of 2010. Five years later, the results of the SAVE $U$ trial were published. This study demonstrated the vaginal sacrospinous hysteropexy to be non-inferior as compared to vaginal hysterectomy in case of uterine descent It [27]. It would be of interest to assess practice pattern variation after the publication of this study, to determine the implementation of this study in the Netherlands and to assess trends in practice pattern variation. We hypothesize that the ratio between hysterectomy and uterus preservation has changed in favour of the hysteropexy and that practice pattern variation will be lower due to more evidence.

\section{LAPAROSCOPIC UTERUS PRESERVING PROCEDURES}

This thesis focuses on laparoscopic sacrohysteropexy whereas the uterus is suspended to the sacral promontory using a mesh. While vaginally-placed mesh has decreased since the FDA notifications, laparoscopic sacrohysteropexy and sacrocolpopexy are considered to be a more durable procedure [25]. The use of abdominal mesh appears to result in lower rates of complications compared with transvaginal mesh [1]. However, long term data is limited for laparoscopic sacrohysteropexy to determine the number of mesh complications. Therefore, it is important to analyse other laparoscopic procedures for uterine suspension without mesh. The best-known laparoscopic procedure without mesh is the laparoscopic uterosacral hysteropexy [57]. This procedure involves placing sutures through the uterosacral ligaments to suspend the cervix with permanent sutures. With regard to this procedure, no large randomized controlled trials are available comparing its effectiveness to vaginal hysterectomy or other hysteropexy procedures. Comparing both laparoscopic hysteropexy techniques is of interest to determine which laparoscopic technique for uterus suspension has the best patient satisfaction and the best functional and anatomical outcome.

Furthermore, apical prolapse can be treated with a laparoscopic sacrocervicopexy. This surgical procedure combines laparoscopic assisted supracervical hysterectomy (LASH) with the suspension of the cervical stump to the sacral promontory using a mesh. Since laparoscopic sacrocervicopexy is not directly compared to laparoscopic sacrohysteropexy, it is unknown if concomitant supracervical hysterectomy leads to better surgical outcome and higher patient satisfaction. It would be interesting to compare these techniques in a RCT.

Finally, the technique of laparoscopic sacrohysteropexy can be optimized. We found practice variation in this surgical technique on several keypoints including extent of dissection, attachment of the mesh to cervix (anterior and/or posterior), and the type of sutures to be used for attachment of the mesh

Well-designed prospective studies or randomized controlled trials are needed to provide evidence for the best surgical technique and to assess to what level that specific technique contributes to surgical outcome. In these studies, specific techniques of the procedure (e.g. extent of dissection, staplers versus tackers to attach the mesh to the promontory) can be compared between study groups. Furthermore, large databases (big data) can contribute to answer these questions as well.

\section{GENERAL CONCLUSION}

According to this thesis, several conclusions can be made:

In case functional and anatomical outcomes after hysterectomy and uterus preserving surgery were equal, more women expressed preference for uterus preservation (43\%) over hysterectomy $(27 \%)$.

In our small country of the Netherlands, we found a high PPV in surgical management of POP and UI with respect to the choice for surgical treatment and the type of surgery. Furthermore, we found great variations in the practice of laparoscopic sacrohysteropexy and sacrocolpopexy, performed by a selected group of gynaecologists.

Laparoscopic sacrohysteropexy is non-inferior to vaginal sacrospinous hysteropexy for surgical failure of the apical compartment at 12 months' follow-up. Following laparoscopic sacrohysteropexy, bothersome overactive bladder and faecal incontinence were more frequent, yet dyspareunia was less frequent 
The higher recurrence risk in the anterior compartment after vaginal sacrospinous hysteropexy cannot be explained by differences in pelvic organ mobility or vaginal axes on dynamic magnetic resonance imaging.

\section{REFERENCES}

1. Maher C, Feiner B, Baessler K, ChristmannSchmid C, Haya N, Brown J. Surgery for women with apical prolapse. Cochrane Database Syst Rev 2018;10:CD012376

2. Glover JA. The incidence of tonsillectomy in school children. Proc R Soc Med 1938; $31: 1219$ 36

3. Weinstein JN, Bronner KK, Morgan TS, Wennberg JE. Trends and geographic variation in major surgery for degenerative diseases of the hip, knee and spine. Health Aff (Millwood) 2004 Suppl Variation: VAR81-9

4. Birkmeyer JD, Reames BN, McCulloch P, Carr AJ, Campbell WB, Wennberg JE. Understanding regional variation in the use of surgery. Lancet 2013;382(9898)1121-9

5. Hanstede MM, Burger MJ, Timmerman A, Burger MP. Regional and temporal variation in hysterectomy rates and surgical routes for benign diseases in The Netherlands. Acta Obstet Gynecol Scand 2012;91:220-5

6. Jones K. Wang $G$, Romano $R$, St Marie $P$ Harmanli O. Colpocleisis: a survey of curren practice patterns. Female Pelvic Med Reconstr Surg 2017;23(4):276-80

7. Detollenaere RJ, Boon J den, Kluivers $K B$, Vierhout ME, Eijndhoven HWF van (2012) Surgical management of pelvic organ prolapse and uterine descent in the Netherlands. Int Urogynecol J;24:781-788

8. Jha S, Cutner A, Moran P. The UK national prolapse survey: 10 years on. Int Urogynecol J 2018:29(6):795-801

9. Wennberg JE, Fisher ES, Skinner JS. Geography and the debate over Medicare reform. Health Aff (Millwood): Supp Web Exclusives: W96-114
10. Elwyn G, Dehlendorf, Epstein RM, Marrin K, White J, Frosch DL. Shared decision making and motivational interviewing achieving patientcentered care across the spectrum of health care problems. Ann Fam Med 2014;12(3):270-5

11. Poprzeczny AJ, Stocking K, Showell M, Duffy JMN. Patient decision aids to facilitate shared decision making in obstetrics and gynecology: a systematic review and meta-analysis. Obstet Gynecol 2020.Doi:10.1097 [E-pub ahead of print]

12. Kennedy AD, Sculpher MJ, Coulter A, Dwyer N, Rees M, Abrams KR et al. Effects of decision aids for menorrhagia on treatment choices, health outcomes, and costs: a randomized controlled trial. JAMA 2002;288(21):2701-8

13. Whelan T, Levine M, Willan A, Gafni A, Sanders K. Effect of a decision aid on knowledge and treatment decision making for breast cancer surgery: a randomized trial. JAMA 2004:292:435-41

14. Auvinen A, Hakama M, Ala-Opas M, Vornanen T. Leppilahti $M$ et al. A randomized trial of choice of treatment in prostate cancer: the effect of intervention on the treatment chosen. BJU Int 2004:93:52-6

15. Fairclough E, Myers J, Smith ARB, Breeman S, Reid F. A UK questionnaire survey of current techniques used to perform pelvic organ prolapse repair. Int Urogynecol J 2017;28(9):1367-76

16. Lensen EJ, Stoutjesdijk JA, Withagen MI, Kluivers KB, Vierhout ME. Technique of anterior colporrhaphy: a Dutch evaluation. Int Urogynecol J 2011;22(5):557-61

17. O'Brien S, Dua A, Vij M. Practices in pelvic organ prolapse operations among surgeons: an 
international survey identifying needs for further research. Int Urogynaecol J 2016;27:1221-26

18. Korbly NB, Kassis NC, Good MM et al. Patient preferences for uterine preservation and hysterectomy in women with pelvic organ prolapse. Am J Obstet Gynecol 2013;209:470. e1-6

19. Frick $A C$, Barber $M D$, Paraiso MF, Ridgeway B, Jelovsek JE, Walters MD. Attitudes toward hysterectomy in women undergoing evaluation for uterovaginal prolapse. Female Pelvic Med Reconstr Surg 2013:19:103-9.

20. Lyatoshinsky P, Fünfgeld C, Popov A, Bezhenar $V$, Krutova V, Ulrich D, Umek W. Pelvic organ prolapse patients $\rrbracket$ attitudes and preferences regarding their uterus: comparing German- and Russian-speaking women. Int Urogynecol J 2019:30(12):2077-83

21. Baskayne K, Willars J, Pitchforth E, Tincello DG. Women's expectations of prolapse surgery: a retrospective quality study. Neurourol Urodyn 2014;33(1):85-9

22. Lawndy SS, Withagen MI, Kluivers KB, Vierhout ME. Between hope and fear: patient's expectations prior to pelvic organ prolapse surgery. Int Urogynecol J 2011;22(9):1159-63

23. DeLancey JO. Anatomic aspects of vaginal eversion after hysterectomy. Am J Obstet Gynecol 1992;166:1717

24. Van IJsselmuiden MN, Detollenaere RJ, Gerritse MBE, Kluivers KB, Bongers MY, van Eijndhoven HWF. Dutch women's attitudes towards hysterectomy and uterus preservation in surgical treatment of pelvic organ prolapse. Eur J Obstet Gynecol Reprod Biol 2018:220:79-83

25. Bradley S, Gutman RE, Richter LA. Hysteropexy: an option for the repair of pelvic organ prolapse. Curr Urol Rep 2018;19(2):15
26. Meriwether KV, Antosh DD, Olivera CK, Kim-Fine $S$, Balk EM, Murphy $M$ et al. Uterine preservation vs hysterectomy in pelvic organ prolapse surgery: a systematic review with meta-analysis and clinical practice guidelines. Am J Obstet Gynecol 2018;219(2):129-46

27. Detollenaere RJ, den Boon J, Stekelenburg J, IntHout J, Vierhout ME, Kluivers KB et al. Sacrospinous hysteropexy versus vaginal hysterectomy with suspension of the uterosacral ligaments in women with uterine prolapse stage 2 or higher: multicentre randomised noninferiority trial. BMJ 2015;351:h3717

28. Schulten SFM, Detollenaere RJ, Stekelenburg J, IntHout J, Kluivers KB, van Eijndhoven HWF. Sacrospinous hysteropexy versus vaginal hysterectomy with uterosacral ligament suspension in women with uterine prolapse stage 2 or higher: observational followup of a multicentre randomized trial. BMJ 2019;366:15149

29. Dietz $\mathrm{V}$, van der Vaart $\mathrm{CH}$, van der Graaf Y, Heintz P. Schraffordt Koops SE. One-year follow-up after sacrospinous hysteropexy and vaginal hysterectomy for uterine descent: a randomized study. Int Urogynecol J 2010;21(2):209-16

30. Zacche MM, Mukhopadhyay S, Giarenis I. Trends in prolapse surgery in England. Int Urogynecol J 2018:29(11):1689-95

31. Price N, Slack A, Jackson SR. Laparoscopic hysteropexy: the initial results of a uterine suspension procedure for uterovaginal prolapse. BJOG 2010;117(1):62-8

32. Rahmanou P, White B, Price N, Jackson S. Laparoscopic hysteropexy: 1- to 4-year followup of women postoperatively. Int Urogynecol J 2014;25(1):131-8

33. Rahmanou P, Price N, Jackson SR. Laparoscopic hysteropexy versus vaginal hysterectomy for the treatment of uterovaginal prolapse: a prospective randomized pilot study. Int Urogynecol J 2015;26(11):1687-94

34. Saldana Ruiz N, Kaiser AM. Fecal Incontinence Challenge and solutions. World J Gastroenterol 2017;7:23(1):11-24

35. Wald A. Diagnosis and management of Faecal Incontinence. Curr Gastroenterol Rep 2018:20.9.

36. Løwenstein E, Møller LA, Laigaard J, Gimbel H Reoperation for pelvic organ prolapse: a Danish cohort study with 15-20 years'follow-up. Int Urogynecol J 2018;29(1):119-124

37. Barber MD, Brubaker L, Nygaard I, Wheeler TL $2^{\text {nd }}$, Schaffer J, Chen Z et al. Defining surgical success after surgery for pelvic organ prolapse. Obstet Gynecol 2009;114:600-9.

38. Petri E, Ashok K. Sacrospinous vaginal fixation - current status. Acta Obstet Gynecol Scand 2011;90:429-36

39. Morgan DM, Rogers MA, Huebner M, Wei JT, Delancey JO. Heterogeneity in anatomic outcome of sacrospinous ligament fixation for prolapse: a systematic review. Obstet Gynecol 2007:109:1424-33

40. Mahran MA, Herath RP, Sayed AT, Oligbo N Laparoscopic management of genital prolapse. Arch Gynecol Obstet 2011;283(5):1015-20

41 Price N, Jackson SR. Advances in laparoscopic techniques in pelvic reconstructive surgery for prolapse and incontinence. Maturitas 2009;62(3):276-80

42. Paraiso MF, Chen CC. Laparoscopic surgery or pelvic organ prolapse. Minerva Gineco 2006:58(5):381-91

43. Coolen AWM, van Oudheusden AMJ, Mol BWJ van Eijndhoven HWF, Roovers JWR, Bongers MY. Laparoscopic sacrocolpopexy compared with open abdominal sacrocolpopexy for vault prolapse repair: a randomized controlled trial. int Urogynecol J 2017;28(10):1469-79

44. Freeman RM, Pantazis K, Thomson A, Frappell J, Bombieri L, Moran P et al. A randomized controlled trial of abdominal versus laparoscopic sacrocolpopexy for the treatment of posthysterectomy vaginal vault prolapse: LAS study. Int Urogynecol J 2013;24(3):377-84

45. Claerhout F, Roovers JP, Lewi P, Verguts J, De Ridder D, Deprest J. Implementation of laparoscopic sacrocolpopexy - a single centre's experience. Int Urogynecol J Pelvic Floor Dysfunct 2009;20(9):1119-25

46. de Mattos Lourenço TR, Pergialiotis V, Durnea C, Elfituri A, Haddad JM, Betschart C, Falconi G, Doumouchtsis SK. A systematic review of reported outcomes and outcome measures in randomized controlled trials on apical prolapse surgery. Int J Gynaecol Obstet. 2019 Apr;145(1):4-11

47. Barber MD, Maher C. Epidemiology and outcome assessment of pelvic organ prolapse. Int Urogynecol J. 2013 Nov;24(11):1783-90

48. Herman MC, Penninx J, Geomini PM, Mol BW, Bongers MY.Choice of primary outcomes evaluating treatment for heavy menstrual bleeding. BJOG. 2016 Sep;123(10):1593-8.

49. Doumouchtsis SK, Pookarnjanamorakot P, Durnea C, Zini M, Elfituri A, Haddad JM, Falconi G, Betschart C, Pergialiotis V. A systematic review on outcome reporting in randomised controlled trials on surgical interventions for female stress urinary incontinence: a call to develop a core outcome set. BJOG. 2019 Nov:126(12):14171422

50. Rada MP, Jones S, Falconi G, Milhem Haddad J, Betschart C, Pergialiotis V, Doumouchtsis 
SK. A systematic review and meta-synthesis of qualitative studies on pelvic organ prolapse for the development of core outcome sets. Neurourol Urodyn. 2020. doi: 10.1002/ nau.24297. [Epub ahead of print]

51. Pergialiotis V, Durnea C, Elfituri A, Duffy J, Doumouchtsis SK. Do we need a core outcome set for childbirth perineal trauma research? A systematic review of outcome reporting in randomised trials evaluating the management of childbirth trauma. BJOG. 2018 Nov:125(12):1522-1531.

52. Healy P, Gordijn S, Ganzevoort W, Beune Baschat A, Khalil A, Kenny L, Bloomfield F, Daly M, Papageorghiou A, Devane D. Core Outcome Set for GROwth restriction: deVeloping Endpoints (COSGROVE). Trials. 2018 Aug 22;19(1):451

53. Rada MP, Pergialiotis $V$, Betschart $C$, Falconi $G$, Haddad JM, Doumouchtsis SK. A protocol fo developing, disseminating, and implementing a core outcome set for stress urinary incontinence. Medicine (Baltimore). 2019 Sep;98(37):e16876

54. Hirsch M, Duffy JM, Barker C, Hummelshoj L, Johnson NP, Mol B, Khan KS, Farquhar C. Protoco for developing, disseminating and implementing a core outcome set for endometriosis. BMJ Open. 2016 Dec 21;6(12):e013998

55. Toozs-Hobson P, Freeman R, Barber M Maher C, Haylen B, Athanasiou S, Swift S, Whitmore K, Ghoniem G, de Ridder D. An International Urogynecological Association (IUGA)/International Continence Society (ICS) joint report on the terminology for reporting outcomes of surgical procedures for pelvic organ prolapse. Neurourol Urodyn. 2012 Apr;31(4):415-21

56. Ellerkmann RM, Cundiff GW, Melick CF, Nihira MA, Leffler K, Bent AE. Correlation of symptoms with location and severity of pelvic organ prolapse. Am J Obstet Gynecol. 2001 Dec;185(6):1332-7

57. Wu MP. Laparoscopic uterine suspension for the treatment of uterovaginal prolapse. Int $\mathrm{J}$ Gynaecol Obstet 1997;59(3):259-60 


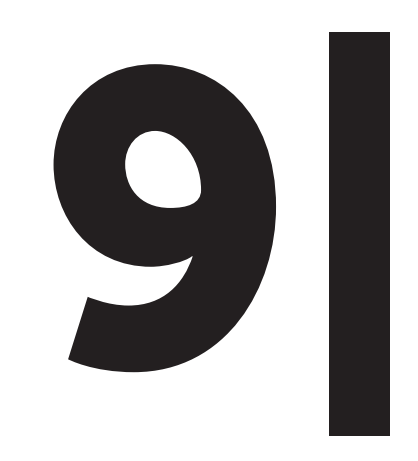

SUMMARY 
This thesis focuses on the surgical management of uterine prolapse. Emphasis is placed on uterine suspension in general and laparoscopic hysteropexy in specific. It comprises the results of the LAVA trial (laparoscopic sacrohysteropexy versus vaginal sacrospinous hysteropexy).

CHAPTER 1 provides a general introduction on pelvic organ prolapse (POP) and the management of uterine prolapse. POP is one of the most common gynaecological disorders. The lifetime risk of undergoing surgery for POP or stress urinary incontinence is estimated to be $20 \%$ by the age of 80 years. Although POP is not a life-threatening condition, it significantly affects a woman's quality of life and may influence urinary, gastrointestinal, sexual and psychological functioning. Traditionally, vaginal hysterectomy was the standard treatment for uterine prolapse but uterine suspension (hysteropexy) is becoming more popular. Although hysteropexy has been compared with hysterectomy in several randomized controlled trials, no randomized controlled trials have been performed comparing different hysteropexy routes so far. As a result, it is unclear whether or not these operations have comparable anatomical and functional outcomes.

In this thesis, the following objectives are addressed:

- How do doctors in the Netherlands treat uterine prolapse? And more specific, how do Dutch gynaecologists perform laparoscopic sacrohysteropexy and sacrocolpopexy?

- What treatment do women in the Netherlands prefer (uterus preservation or removal) when surgical management for uterine descent is indicated?

- How should women with uterine prolapse be treated and is there a difference in outcomes between laparoscopic and vaginal hysteropexy?

- Is there a difference in pelvic mobility after sacrospinous hysteropexy as compared to other treatments for uterine prolapse?

CHAPTER 2 describes practice pattern variation (PPV) in treatment of POP and urinary incontinence. The availability of many treatment modalities for POP can lead to variation in care between physicians and hospitals, the so-called PPV. PPV is defined as the difference in care that cannot be explained by the underlying medical condition. In this chapter we describe PPV in Dutch hospitals in treatment of POP and urinary incontinence. A high PPV per hospital and per region was found. In some hospitals, a hysterectomy was performed in all cases of uterovaginal prolapse, while in other hospitals uterus-preserving techniques were mostly performed. Furthermore, a high PPV of transvaginal mesh placement was found. In the small country of the
Netherlands, a patient's odds of undergoing surgery for POP and urinary incontinence seems to depend more on where the patient lives and to which hospital she is referred than on clinical circumstances. The choice of treatment is mainly subjected to the preference of the gynaecologists. The absence of clearly defined guidelines regarding indications for conservative and surgical management of POP and the availability of different surgical techniques might contribute to this high PPV.

CHAPTER 3 focuses on variation in practice of laparoscopic sacrohysteropexy and laparoscopic sacrocolpopexy. A standard approach or published guideline on how to perform these procedures is lacking. Different methods have been described in literature, and there is no consensus on how to perform these procedures. In this chapter we describe the variation in surgical technique of laparoscopic sacrohysteropexy and laparoscopic sacrocolpopexy among Dutch gynaecologists. A high practice variation was found on several key points, such as level of dissection along the anterior and posterior walls of the vagina, the type of mesh, the type of sutures, the tension of the implanted mesh and reperitonealisation of the mesh. To monitor the outcome and long-term complications of laparoscopic prolapse repair, consensus on how to perform these procedures is mandatory.

CHAPTER 4 presents the results of a prospective study regarding women's preferences towards hysterectomy or uterus preservation. It is believed that uterus preservation is becoming more popular because women want to retain their uterus more often. However, evidence to support this statement is lacking. In this chapter women's attitudes towards hysterectomy and uterus preservation in surgical management of uterine prolapse are described. In case outcomes after hysterectomy and uterus preserving surgery were expected to be equal, more women expressed preference for uterus preservation $(43 \%, 44 / 102$ women) compared to hysterectomy $(27 \%$, $27 / 102$ women). The majority of women expected that sexuality and body image would equally improve after both interventions. Treatment success, risk of urinary incontinence after surgery and complication risk were the most important factors for choosing a treatment. Taken the future risk of endometrial cancer into account, 18\% of the women preferred hysterectomy because of this risk.

CHAPTER 5 provides a study protocol for a randomised clinical trial to investigate outcomes after laparoscopic sacrohysteropexy and vaginal sacrospinous hysteropexy in women with uterine prolapse stage 2 or higher (LAVA-trial). The study was designed to test the hypothesis that laparoscopic sacrohysteropexy was non-inferior to vaginal sacrospinous hysteropexy regarding surgical failure at 12 months' follow-up. Primary 
outcome of this study was surgical failure, defined as either bothersome symptoms and/or repeat surgery for prolapse stage 2 or higher at point $C$ by 12 months postoperative. Secondary outcomes were overall anatomical recurrences, functional outcome, quality of life, complications, hospital stay, post-operative recovery, and sexual functioning. All women with uterine prolapse POP-Q stage 2 or higher without previous POP surgery are eligible for participation. Exclusion criteria are a known malignancy of the cervix or cervical dysplasia, language barriers, a wish to preserve fertility, presence of immunological or hematological disorders interfering with recovery after surgery, contraindications for laparoscopic surgery (e.g. ileus, risk of severe adhesions), abnormal ultrasound findings of uterus or ovaries which cause symptoms and/or require treatment, abnormal uterine bleeding that requires surgical treatment, postmenopausal bleeding in the past year and women who are unwilling to return for follow-up.

According to the power analysis, a total of 124 women (62 in each arm) were needed to prove the hypothesis.

CHAPTER 6 presents the results of the LAVA trial. In total, 126 women with uterine prolapse stage two or higher requiring surgery, were randomised between laparoscopic sacrohysteropexy $(n=64)$ and vaginal sacrospinous hysteropexy $(n=62)$ in one of the six participating hospitals. Laparoscopic sacrohysteropexy was non-inferior to vaginal sacrospinous hysteropexy regarding surgical failure after 12 months in the intention to treat analysis. The failure rate was $1 / 64(1.6 \%)$ in the laparoscopic group versus $2 / 62(3.3 \%)$ in the vaginal group (difference $-1.7 \% ; 95 \% \mathrm{Cl}$ for difference -7.1 to 3.7 ). A per protocol analysis also demonstrated non-inferiority of the laparoscopic sacrohysteropexy. There were no differences in overall anatomical recurrences and quality of life. More bothersome symptoms of overactive bladder and faecal incontinence were reported after laparoscopic sacrohysteropexy. Dyspareunia was more frequently reported after vaginal sacrospinous hysteropexy. Based on the analysis after 12 months' followup, we concluded that laparoscopic sacrohysteropexy was non-inferior to vaginal sacrospinous hysteropexy for surgical failure of the apical compartment.

CHAPTER 7 describes the results of a prospective study regarding pelvic organ mobility and vaginal axes assesses by dynamic magnetic resonance imaging (MRI) after different surgical procedures for uterine descent. In literature, the high risk for recurrent prolapse of the anterior vaginal wall after vaginal sacrospinous hysteropexy is often discussed. In order to find an explanation, the vaginal axes and pelvic organ mobility after vaginal sacrospinous hysteropexy were studied by dynamic MRI and compared to other surgical techniques for uterine descent (laparoscopic sacrohysteropexy and vaginal hysterectomy with uterosacral suspension). No difference in pelvic organ mobility was found 6 months after vaginal sacrospinous hysteropexy as compared to vaginal hysterectomy and laparoscopic sacrohysteropexy. After vaginal hysterectomy, we found a more posterior position of the upper vagina as compared to vaginal sacrospinous hysteropexy and laparoscopic sacrohysteropexy. Based on these data, the higher recurrence risk in the anterior compartment after vaginal sacrospinous hysteropexy cannot be explained.

CHAPTER 8 discusses the main results of this thesis, its clinical implication and topics for future research. According to this thesis, several conclusions can be made:

- In case functional and anatomical outcomes after hysterectomy and uterus preserving surgery were equal, more women expressed preference for uterus preservation (43\%) over hysterectomy (27\%).

- In our small country of the Netherlands, we found a high PPV in surgical management of POP and $\mathrm{UI}$ with respect to the choice for surgical treatment and the type of surgery. Furthermore, we found great variations in the practice of laparoscopic sacrohysteropexy and sacrocolpopexy, performed by a selected group of gynecologists.

- Laparoscopic sacrohysteropexy is non-inferior to vaginal sacrospinous hysteropexy for surgical failure of the apical compartment at 12 months' follow-up. Following laparoscopic sacrohysteropexy, bothersome overactive bladder and faecal incontinence were more frequent, yet dyspareunia was less frequent

- The higher recurrence risk in the anterior compartment after vaginal sacrospinous hysteropexy cannot be explained by differences in pelvic organ mobility or vaginal axes on dynamic magnetic resonance imaging. 


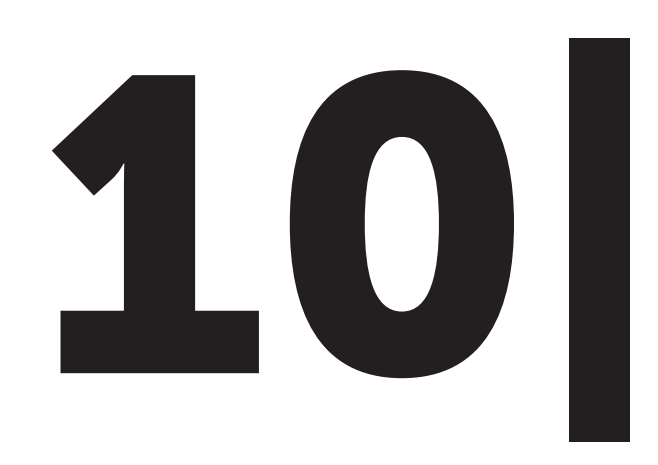

NEDERLANDSE SAMENVATTING 
Dit proefschrift richt zich op de operatieve behandeling van uterusprolaps (baarmoederverzakking) bij vrouwen. De nadruk ligt op uterussparende prolapschirurgie (hysteropexie) in het algemeen, en op laparoscopische hysteropexie in het bijzonder. Het beschrijft de resultaten van de LAVA-studie (LAparoscopische sacrohysteropexie versus VAginale sacrospinale hysteropexie).

HOOFDSTUK 1 geeft een algemene introductie over (uterus)prolaps en de behandeling van uterusprolaps. Een prolaps is een veelvoorkomende aandoening. Het levenslange risico om geopereerd te worden vanwege prolaps is $20 \%$ op de leeftijd van 80 jaar. Alhoewel een prolaps niet levensbedreigend is, heeft het een duidelijk negatieve invloed op de kwaliteit van leven van de vrouw, maar ook op seksueel en psychisch functioneren. Tevens kunnen er klachten ontstaan op het gebied van mictie en defaecatie.

Van oudsher was het verwijderen van de uterus de standaardbehandeling voor uterusprolaps, maar uterussparende chirurgie wordt steeds populairder. Verschillende randomized controlled trials hebben hysteropexie vergeleken met vaginale hysterectomie, maar er zijn tot op heden nog geen studies uitgevoerd die de verschillende technieken voor hysteropexie met elkaar vergelijken. Hierdoor is het onbekend hoe de anatomische en functionele uitkomsten van deze verschillende operaties ten opzichte van elkaar zijn

In dit proefschrift worden de volgende onderwerpen behandeld:

- Hoe wordt prolaps door Nederlandse gynaecologen behandeld? En specifieker, hoe voeren Nederlandse gynaecologen een laparoscopische sacrohysteropexie en sacrocolpopexie uit?

- Welke behandeling heeft de voorkeur voor Nederlandse vrouwen (uterussparende chirurgie of hysterectomie) wanneer een chirurgische behandeling voor uterusprolaps is geïndiceerd?

- Hoe moeten vrouwen met uterusprolaps behandeld worden en is er verschil in uitkomst tussen vaginale en laparoscopische hysteropexie?

- Is er een verschil in mobiliteit van de bekkenorganen na sacrospinale hysteropexie vergeleken met andere operaties voor uterusprolaps?

In HOOFDSTUK 2 bestudeerden we de praktijkvariatie in Nederlandse ziekenhuizen in de behandeling van prolaps en urine-incontinentie. De verschillende behandelmogelijkheden voor prolaps kunnen leiden tot variatie in behandeling tussen artsen en tussen ziekenhuizen (praktijkvariatie). Praktijkvariatie wordt gedefinieerd als de variatie in behandeling die niet verklaard kan worden door de onderliggende aandoening. We vonden een grote praktijkvariatie per ziekenhuis en per regio. In sommige ziekenhuizen werd altijd een hysterectomie verricht in geval van een chirurgische behandeling voor uterusprolaps, terwijl in andere ziekenhuizen bijna altijd een uterussparende operatie werd uitgevoerd. Een grote praktijkvariatie werd ook gezien in het plaatsen van kunststofmaterialen. In een klein land als Nederland is de kans voor een patiënt om geopereerd te worden aan een verzakking of urineincontinentie meer afhankelijk van waar ze woont en naar welk ziekenhuis ze wordt verwezen dan van de klinische omstandigheden. De keuze van de behandeling wordt vooral bepaald door de gynaecoloog. De geconstateerde praktijkvariatie zou kunnen komen door afwezigheid van een duidelijke richtlijn over de chirurgische behandeling van prolaps

HOOFDSTUK 3 beschrijft de variatie in operatietechniek van laparoscopische sacrohysteropexie en laparoscopische sacrocolpopexie. Een standaard beschrijving, protocol of richtlijn hoe deze ingrepen uit te voeren ontbreekt. Verschillende methoden zijn beschreven in de literatuur, maar er is tot op heden geen consensus over hoe de ingrepen uit te voeren. In dit hoofdstuk beschrijven we de variatie in operatietechniek van de laparoscopische sacrohysteropexie en laparoscopische sacrocolpopexie tussen Nederlandse gynaecologen. Een grote praktijkvariatie werd gevonden op enkele belangrijke punten in de operatie, zoals niveau van dissectie langs de anterieure en posterieure vaginawanden, het type kunststofmateriaal (mesh), het type hechtingen, de spanning van de mesh en reperitonealisatie van de mesh. Het is erg belangrijk om consensus te verkrijgen, hoe de ingrepen uit te voeren, zodat uitkomsten en lange-termijn complicaties in beeld kunnen worden gebracht.

HOOFDSTUK 4 presenteert de resultaten van een prospectieve studie naar de voorkeur en houding van Nederlandse vrouwen ten aanzien van uterussparend opereren en hysterectomie. In de literatuur wordt vaak beweerd dat behouden van de uterus steeds populairder wordt en dat vrouwen steeds vaker hun uterus niet willen laten verwijderen als chirurgische behandeling nodig is. Echter het bewijs voor deze laatste bewering is beperkt. In dit hoofdstuk wordt de preferentie van Nederlandse vrouwen beschreven. Wanneer de uitkomsten na een hysterectomie en uterussparende operatie gelijk zouden zijn, hadden meer vrouwen een voorkeur voor het behouden van de uterus $(43 \%, 44 / 102$ vrouwen) vergeleken met een hysterectomie $(27 \%, 27 / 102$ vrouwen). De meerderheid van de vrouwen verwachtte een gelijke verbetering in seksualiteit en lichaamsbeeld na beide behandelmethoden. Behandelsucces, risico op urine-incontinentie en het risico op complicaties werden het belangrijkst bevonden. 
Het toekomstig risico op een endometriumcarcinoom was voor $18 \%$ van de vrouwen reden om te kiezen voor een hysterectomie.

In HOOFDSTUK $\mathbf{5}$ beschrijven we het ontwerp van de LAVA-studie. Dit is een gerandomiseerde studie waarin de laparoscopische sacrohysteropexie wordt vergeleken met de vaginale sacrospinale hysteropexie bij vrouwen met een uterusprolaps stadium 2 of hoger. De studie werd ontworpen om de hypothese te testen dat de laparoscopische sacrohysteropexie non-inferieur is aan de vaginale sacrospinale hysteropexie na 12 maanden follow-up. De primaire uitkomst was chirurgisch falen, gedefinieerd als recidief stadium 2 of hoger van de uterus (het apicale compartiment) vastgesteld middels POP-Q onderzoek, met daarbij hinderlijke prolapsklachten of een reïnterventie (operatie of pessarium) vanwege recidief prolaps van het apicale compartiment. Secundaire uitkomstmaten waren recidief prolaps in alle compartimenten, functionele uitkomsten, kwaliteit van leven, complicaties, opnameduur, postoperatief herstel en seksueel functioneren. Alle vrouwen met een uterusprolaps POP-Q stadium 2 of hoger zonder voorafgaande prolapsbehandeling werden gevraagd deel te nemen

Exclusie criteria waren bekende maligniteit of dysplasie van de cervix, een taalbarrière, wens tot behoud fertiliteit, aanwezigheid van immunologische of hematologische aandoeningen die het postoperatieve herstel kunnen beïnloeden, contra-indicaties voor laparoscopie (zoals ileus, verdenking op ernstige adhesies), afwijkende bevindingen bij echografisch onderzoek aan uterus en/of ovaria die klachten veroorzaken of waarvoor een behandeling nodig is, abnormaal vaginaal bloedverlies wat chirurgische behandeling behoeft, postmenopauzaal bloedverlies in het afgelopen jaar en niet terug willen komen voor follow-up.

Volgens de powerberekening moesten er 124 vrouwen (62 per studie arm) geïncludeerd worden om de hypothese te kunnen testen.

In HOOFDSTUK 6 presenteren we de resultaten van de LAVA-studie. In totaal werden 126 vrouwen met een uterusprolaps stadium 2 of hoger gerandomiseerd tussen laparoscopische sacrohysteropexie $(n=64)$ en vaginale sacrospinale hysteropexie $(n=62)$ in één van de zes deelnemende ziekenhuizen. De intention to treat analyse liet zien dat de laparoscopische sacrohysteropexie non-inferieur was aan de vaginale sacrospinale hysteropexie wat betreft chirurgisch falen van het apicale compartiment na 12 maanden follow-up. Chirurgisch falen werd gezien bij 1.6\% (1/64) van de vrouwen na een laparoscopische sacrohysteropexie tegenover $3.3 \%(2 / 62)$ van de vrouwen na een vaginale sacrospinale hysteropexie (verschil -1.7\%; 95\% BI -7.1 tot 3.7). Per protocol analyse liet ook zien dat een laparoscopische sacrohysteropexie noninferieur was. Er was geen verschil in het totaal aantal recidieven en in kwaliteit van leven. $\mathrm{Na}$ een laparoscopische sacrohysteropexie werden meer hinderlijke klachten van overactieve blaas en faecale incontinentie gemeld. Dyspareunie werd frequenter gerapporteerd na een vaginale sacrospinale hysteropexie. Op basis van de analyse na 12 maanden concluderen wij dat een laparoscopische sacrohysteropexie noninferieur is aan een vaginale sacrospinale hysteropexie wat betreft recidief prolaps van het apicale compartiment met hinderlijke prolapsklachten en/of herbehandeling (operatie of pessarium) vanwege prolaps van het middelste compartiment.

HOOFDSTUK 7 beschrijft de resultaten van een prospectieve studie naar de mobiliteit van de bekkenorganen en vaginale as na verschillende chirurgische behandelingen voor uterusprolaps. In literatuur wordt het hoge risico op een voorwandprolaps na vaginale sacrospinale hysteropexie vaak beschreven. Om een oorzaak voor dit hoge recidiefaantal te vinden, hebben we vaginale assen en de mobiliteit van de bekkenorganen in kaart gebracht met behulp van een dynamische MRI. We hebben hiervoor vrouwen na een vaginale sacrospinale hysteropexie vergeleken met vrouwen na een laparoscopische sacrohysteropexie of een vaginale hysterectomie met uterosacrale suspensie. Zes maanden na de operatie werd geen verschil gevonden in mobiliteit van de bekkenbodemorganen na vaginale sacrospinale hysteropexie vergeleken met vaginale hysterectomie en laparoscopische sacrohysteropexie. $\mathrm{Na}$ vaginale hysterectomie is de as van het bovenste deel van de vagina meer naar posterior gelegen in vergelijking met de uterussparende ingrepen. Op basis van deze data kunnen we geen verklaring geven voor het hoge recidiefrisico van het anterieure compartiment na een vaginale sacrospinale hysteropexie.

HOOFDSTUK 8 bespreekt de resultaten van dit proefschrift, de klinische implicaties en de onderwerpen voor toekomstig onderzoek. Aan de hand van dit proefschrift kunnen de volgende conclusies getrokken worden:

- Wanneer de functionele en anatomische uitkomsten van hysterectomie en uterussparende chirurgie gelijk zijn, hebben meer vrouwen een voorkeur voor uterussparende chirurgie $(43 \%)$ ten opzichte van de hysterectomie $(27 \%)$.

- In een klein land als Nederland vonden we een hoge praktijkvariatie in de chirurgische behandeling van prolaps en urine-incontinentie met betrekking tot chirurgische versus conservatieve behandeling en soort chirurgische behandeling. We vonden tevens variaties in de gebruikte techniek van laparoscopische sacrohysteropexie en laparoscopische sacrocolpopexie, uitgevoerd door een 
selecte groep gynaecologen.

- Laparoscopische sacrohysteropexie is non-inferieur aan vaginale sacrospinale hysteropexie wat betreft chirurgisch falen in het apicale compartiment na 12 maanden follow-up. Na laparoscopische sacrohysteropexie worden meer hinderlijke klachten van overactieve blaas en faecale incontinentie gemeld, maar dyspareunie komt minder frequent voor.

- Het verhoogde risico op een voorwandprolaps na een vaginale sacrospinale hysteropexie kan niet worden verklaard door verschillen in mobiliteit van de bekkenorganen of vaginale as, bepaald met behulp van dynamische MRI. 


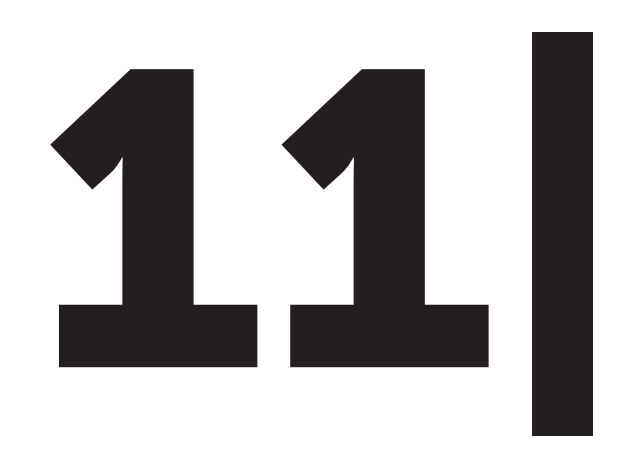

VALORISATION ADDENDUM 


\section{WHY IS THIS THESIS RELEVANT?}

Traditionally, uterine prolapse is treated by vaginal hysterectomy and suspension of the vaginal vault. However, nowadays more women express a preference for uterus preservation and consequently, hysteropexy techniques are gaining interest. Several procedures have been described to suspend the uterus, including the vaginal or the abdominal (laparoscopic/robotic) route. Although the different techniques for hysteropexy have been studied for many years, no randomised controlled trials have been performed to compare these techniques. As a result, it is unclear whether or not these uterine suspension procedures have comparable anatomical and functional outcomes. This thesis focuses on the effectiveness of laparoscopic hysteropexy compared to vaginal hysteropexy. Furthermore, variation in treatment of pelvic organ prolapse (POP) is assessed.

\section{RELEVANCE}

POP is one of the most common gynaecological conditions. The aetiology of POP is multifactorial. Risk factors include higher parity, vaginal childbirth, advancing age, obesity and previous hysterectomy.

Although POP is not a life-threatening condition, it significantly affects a woman's quality of life and may influence urinary, gastrointestinal, sexual and psychological functioning. The lifetime risk of undergoing surgery for POP or stress urinary incontinence is estimated to be $20 \%$ by the age of 80 years. In the Netherlands, about 13.000 POP surgeries are performed every year, with estimated total costs of 75 million euro per year. Due to aging of the population, a significant increase in both the number of women with POP and those seeking care for POP is expected to occur over the next decades. As a result, there will be an enormous extra demand for future prolapse treatment.

\section{PRACTICE PATTERN VARIATION}

In the Netherlands, a great variation was found in the treatment of POP. The surgical rate of POP and urinary incontinence (UI) varies per hospital and per region. Consequently, a woman's odd of undergoing surgery for POP seems to depend more on where she lives than on the clinical circumstances.

Practice pattern variation in the ratio between conservative and surgical treatment of POP might imply over- and undertreatment. In case of undertreatment, the patient might not receive adequate treatment. However, in case of overtreatment the patient is exposed to the unnecessary risks of surgery. Since a gold standard for treating POP is still a matter of debate, the main question arises what the optimal ratio is between conservative and surgical treatment management.

To reduce practice pattern variation, well-designed prospective studies are necessary to establish evidence based guidelines.

\section{HYSTEROPEXY}

Vaginal sacrospinous hysteropexy is the most studied surgical technique for uterine descent in the Netherlands and the procedure is very popular among Dutch gynaecologists. However, there are some concerns about this technique. After vaginal sacrospinous hysteropexy, the vaginal axis is changed to a more horizontal and posterior position. Furthermore, the high risk of anterior vaginal wall prolapse after this technique is often discussed. Abdominal or laparoscopic uterine suspension might not have these disadvantages. In case of vaginal vault prolapse, abdominal sacrocolpopexy has been shown to have a lower rate of recurrent apical prolapse and a lower reoperation rate as compared to vaginal sacrospinous fixation of the vaginal vault.

In our randomised controlled study (LAVA-trial) we compared laparoscopic sacrohysteropexy with vaginal sacrospinous hysteropexy in women with uterine descent POP-Q stage 2 or higher. To our knowledge, this is the first randomised controlled trial comparing these two techniques for hysteropexy. The study demonstrated non-inferiority of laparoscopic sacrohysteropexy as compared to vaginal sacrospinous hysteropexy for surgical failure in the apical compartment. Furthermore, no differences were found in anatomical and surgical failure in other compartments, neither in sexual functioning and quality of life. However, we found some differences in secondary outcomes. Following laparoscopic sacrohysteropexy, bothersome symptoms of overactive bladder and faecal incontinence were reported more frequently, but dyspareunia was reported less frequent. These subtle differences in secondary outcomes may help in the process of shared-decision making and choose the optimal surgical route for a specific patient.

\section{TARGET GROUPS}

The results of this thesis are interesting for physicians, gynaecologists, general practitioners, medical industry and most important, women. The study regarding practice pattern variation demonstrates great variation in the treatment of POP in a small country as the Netherlands, and indicates the importance of developing a clearly defined guideline. 
The results of the LAVA-trial will not only help to establish evidence-based guidelines, but also help to develop a decision tool for shared-decision making. Hopefully this will lead to an increase in patient satisfaction.

\section{ACTIVITIES AND INNOVATIONS}

All study results have been submitted to international scientific research journals. To gain more attention on this work, the results of the studies are presented and discussed on national and international scientific meetings.

\section{SCHEDULE AND IMPLEMENTATION}

In order to evaluate treatments for POP and to determine the best treatment with the highest patient satisfaction, further research is necessary. Studies regarding conservative versus surgical treatment of POP are needed, and furthermore the technique of laparoscopic sacrohystopexy can be optimized. Other laparoscopic uterine suspension techniques need to be evaluated as well.

One major problem in evaluating effects of POP surgery is the range of outcome measures, which are not reported in a consistent manner. As a result of heterogenous outcomes, meaningful meta-analysis of POP outcome data is impossible. Regarding POP surgery, any definition of success should include the absence of vaginal bulge symptoms in addition to anatomical criteria and the absence of re-treatment. However, patient reported outcome measurements (PROM's) on quality of life, functional outcome and sexual functioning are perhaps the most important since severity of the prolapse does not correlate with prolapse symptoms or the impact on quality of life. In addition, the goals of POP treatment are reducing the symptoms and improving quality of life. Therefore, these PROM's should always take into account when evaluating surgical outcomes. 


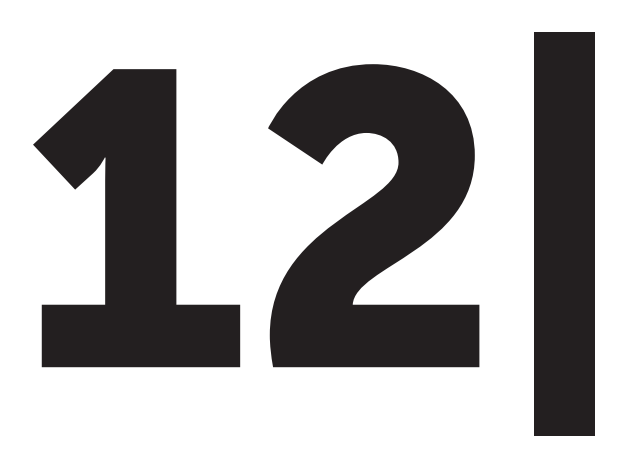

LIST OF ABBREVIATIONS

QUESTIONNAIRES

PUBLICATIONS

DANKWOORD

CURRICULUM VITAE 


\section{LIST OF ABBREVIATIONS}

$\mathrm{BMI}$

$\mathrm{Cl}$

DDI

EQ5D

FDA

IIQ

ITT

ITT-LOCF

LSC

$\mathrm{LSH}$

MR

$\mathrm{OAB}$

PFMT

PISQ

$P O P$

$P O P-Q$

$P P$

PPV

PROM

RCT

SDM

SF-36

SSHP

$\mathrm{VH}$

Body Mass Index

Confidence interval

Defecatory Distress Inventory

Euroqol-5D

Food and Drug Administration

Incontinence Impact Questionnaire

Intention to treat

Intention to treat with last observation carried forward

Laparoscopic sacrocolpopexy

aparoscopic sacrohysteropexy

Magnetic Resonance Imaging

Overactive bladder

Pelvic floor muscle training

Pelvic organ prolapse/Urinary incontinence Sexual questionnaire

Pelvic organ prolapse

Pelvic organ prolapse quantification system

Per protocol

Practice pattern variation

Patient reported outcome measurements

Randomised controlled trial

Shared-decision making

Short Form-36

Sacrospinous hysteropexy

Urinary incontinence

Vaginal hysterectomy 


\section{VRAGENLIJST PREFERENTIE STUDIE}

\section{STEL DAT U EEN BAARMOEDERVERZAKKING HEBT}

1. Stel er is helemaal geen verschil tussen het verwijderen of het sparen van de baarmoeder. De herstelperioden zijn even lang, de kans op opnieuw een verzakking is even groot en de klachten na de ingreep zijn hetzelfde. Welke behandeling heeft dan op dit moment uw voorkeur?

$\square$ Baarmoederverwijdering

$\square$ Baarmoedersparende ingreep

$\square$ Geen voorkeur

Indien u een voorkeur aangeeft: waarom kiest u voor deze ingreep?

De belangrijkste risico's van opereren bij een verzakking zijn een nabloeding, infectie en een beschadiging van de blaas of de darm. Ook kunnen er tijdelijke problemen zijn met uitplassen. Deze risico's lijken op dit moment bij beide type operaties even groot.

Sommige vrouwen krijgen na de operatie opnieuw een verzakking. Zowel na een baarmoedersparende ingreep als na het verwijderen van de baarmoeder kan er opnieuw een verzakking ontstaan. Indien de baarmoeder is verwijderd, is er sprake van een verzakking van de top van de schede. Op dit moment is het niet duidelijk of het opnieuw optreden van een verzakking vaker voorkomt bij het verwijderen van de baarmoeder of bij een baarmoedersparende ingreep, omdat dit nog weinig is onderzocht.

Het is ook mogelijk dat de operatie helpt tegen uw klachten, maar dat $u$ er andere klachten voor in de plaats krijgt. Een klein aantal vrouwen krijgt bijvoorbeeld last van ongewenst urineverlies (incontinentie) na een operatie voor een verzakking. Mogelijk gaat het verwijderen van de baarmoeder iets vaker gepaard met ongewenst urineverlies na de operatie in vergelijking met baarmoedersparende operaties, maar ook dit moet nog meer onderzocht worden.

Uit eerder onderzoek blijkt dat sommige baarmoedersparende operaties gepaard gaan met een kortere opnameduur in het ziekenhuis na de operatie (ongeveer een dag korter) en een iets sneller herstel na de ingreep. 
2. Na het lezen van de bovenstaande informatie kies ik

$\square$ Voor dezelfde ingreep namelijk. de baarmoederverwijdering

$\square$ Voor de andere ingreep namelijk de baarmoederverwijdering

$\square$ Voor dezelfde ingreep namelijk de baarmoedersparende ingreep

$\square$ Voor de andere ingreep namelijk de baarmoedersparende ingreep

$\square$ Ik heb nog steeds geen voorkeur

Indien u gekozen hebt voor een andere ingreep: waarop is dit gebaseerd?

3. De gemiddelde opnameduur na een operatie vanwege een baarmoederverzakking is 2-6 dagen. Bij hoeveel dagen verschil in opnameduur zou u toch kiezen voor de andere operatie? Dus hoeveel dagen moet de opnameduur korter zijn, wil u voor de andere ingreep kiezen?
$\square 1$ dag
$\square 2$ dagen
$\square 3$ dagen
$\square 4$ dagen
$\square$ Meer dan 4 dagen
$\square$ Ik verander hierdoor niet van keuze

4. Het gemiddelde herstel na een verzakkingsoperatie (geheel herstel, weer aan het werk) duurt ongeveer 6 tot 10 weken. Bij welk verschil in het aantal weken herstel zou u voor de andere ingreep kiezen ten opzichte van uw keuze bij vraag 2? Dus hoeveel weken moet het herstel korter zijn om toch voor de andere ingreep te kiezen?
$\square$ minder dan 1 week
$\square 1$ week
$\square 2$ weken
$\square 4$ weken
$\square 6$ weken
$\square$ Ik verander hierdoor niet van keuze

Urine-incontinentie kan optreden na een operatieve ingreep. Hierbij kan onderscheid gemaakt worden in aandrangincontinentie (bij aandrang om te plassen ontstaat ongewenst urineverlies) en incontinentie bij drukverhogende momenten zoals hoesten, niezen en persen (stressincontinentie).

5. Hieronder staan geschatte percentages van optreden van aandrang urineincontinentie na de door uw gekozen procedure. Vanaf welk verschil in risico op aandrang urine-incontinentie zou $\mathrm{u}$ voor de andere ingreep kiezen ten opzichte van uw keuze bij vraag 2?
$\square$ 5\%
(5 op 100 vrouwen)
$\square \quad 10 \%$
$\square 15 \%$
$\square 20 \%$
$25 \%$
$\square 30 \%$
$\square 35 \%$
$\square \quad 40 \%$
$\square 45 \%$
$\square 50 \%$
(10 op de 100 vrouwen)
(15 op de 100 vrouwen)
(20 op de 100 vrouwen)
(25 op de 100 vrouwen)
(30 op de 100 vrouwen)
(35 op de 100 vrouwen)
(40 op de 100 vrouwen)
(45 op de 100 vrouwen)
$\square$ Meer dan $50 \%$
$\square \quad$ Ik kies niet voor een andere ingreep

6. Hieronder staan geschatte percentages van optreden van stressincontinentie (ongewenst urineverlies bij drukverhogende momenten, zoals niezen, persen en hoesten) na de door uw gekozen procedure. Vanaf welk verschil in risico op stressincontinentie zou $u$ voor de andere ingreep kiezen ten opzichte van uw keuze bij vraag 2?

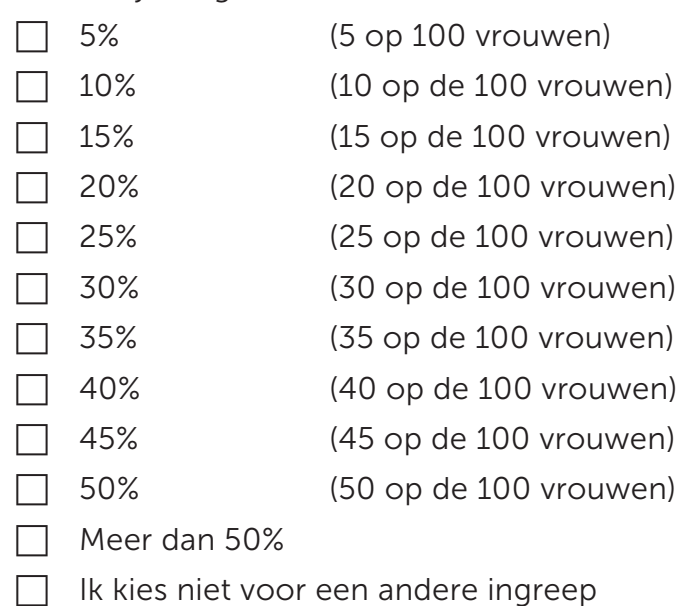


7. Geef hieronder aan op een schaal van 1 tot 5 hoe belangrijk $u$ de onderstaande factoren vindt in het maken van een keuze tussen de twee ingrepen (baarmoedersparende operatie of baarmoederverwijdering).

\begin{tabular}{lccccc}
\hline & $\begin{array}{l}\text { Helemaal niet } \\
\text { belangrijk }\end{array}$ & Niet belangrijk & Neutraal & Belangrijk & $\begin{array}{c}\text { Heel erg } \\
\text { belangrijk }\end{array}$ \\
\hline Verzakking weg & $\square$ & $\square$ & $\square$ & $\square$ & $\square$ \\
Risico op ongewenst urineverlies na & $\square$ & $\square$ & $\square$ & $\square$ & $\square$ \\
de ingreep & & & & & \\
Herstel na de operatie & $\square$ & $\square$ & $\square$ & $\square$ & $\square$ \\
Kosten & $\square$ & $\square$ & $\square$ & $\square$ & $\square$ \\
$\begin{array}{l}\text { Duur van de opname } \\
\text { Complicaties na de ingreep }\end{array}$ & $\square$ & $\square$ & $\square$ & $\square$ & $\square$ \\
(nabloeding, infectie, problemen met & $\square$ & $\square$ & $\square$ & $\square$ & $\square$ \\
uitplassen) & & & & & \\
Seksueel functioneren na de ingreep & $\square$ & $\square$ & $\square$ & $\square$ & $\square$ \\
\hline
\end{tabular}

8. Denkt $u$ dat het type operatie van invloed is op de mate van verandering in uw lichaamsbeeld / zelfbeeld na de operatie?

$\square$ Nee

$\square$ Ja, mijn lichaamsbeeld/zelfbeeld zal meer verbeteren door een baarmoederverwijdering

$\square$ Ja, mijn lichaambeeld/zelfbeeld zal meer verbeteren door een baarmoedersparende operatie

9. Denkt $u$ dat het type operatie van invloed is op de mate van verandering in seksualiteit na de operatie?

$\square$ Nee

$\square$ Ja, mijn seksualiteit zal meer verbeteren door een baarmoederverwijdering

$\square$ Ja, mijn seksualiteit zal meer verbeteren door een baarmoedersparende operatie
10. Wanneer een baarmoederverwijdering wordt uitgevoerd, kan er geen baarmoederslijmvlieskanker ontstaan. Wanneer een baarmoedersparende operatie wordt uitgevoerd, is er een kleine kans op het krijgen van baarmoederslijmvlieskanker. Deze kans is NIET toegenomen door de ingreep. In Nederland wordt jaarlijks bij ongeveer 1900 vrouwen baarmoederslijmvlieskanker gediagnosticeerd. Deze vorm van kanker wordt vaak in een vroege fase ontdekt doordat vrouwen abnormaal vaginaalbloedverlies hebben. Bij dit vroege stadium bedraagt de globale 5 -jaarsoverleving $95 \%$. Dit betekent dat $95 \%$ van de vrouwen waarbij in dit stadium baarmoederslijmvlieskanker is geconstateerd nog in leven is. Beïnvloeden deze gegevens uw keuze voor een ingreep?
$\square$ Nee
$\square \mathrm{Ja}$
$\square$ Weet ik niet

11. De meeste vrouwen hebben een verbetering van hun seksleven na een verzakkingsoperatie. Sommige vrouwen hebben na een operatie vanwege verzakking pijn bij vrijen, wat soms blijvend kan zijn. Vanaf welk verschil in risico op pijn tijdens het vrijen zou u voor de andere ingreep kiezen ten opzichte van uw keuze bij vraag 2 ?
$\square 5 \%$
$\square \quad 10 \%$
$\square 15 \%$
$\square \quad 20 \%$
$\square \quad 25 \%$
$\square \quad 30 \%$
$\square 35 \%$
$\square \quad 40 \%$
$\square 45 \%$
$\square 50 \%$
$\square \quad$ Meer dan $50 \%$
$\square \quad \mathrm{Ik}$ kies niet voor een andere ingreep 
12. Sommige vrouwen krijgen in de loop van hun leven vloeistoornissen look wel abnormaal vaginaal bloedverlies genoemd), bijvoorbeeld zeer hevig bloedverlies tijdens de menstruatie of onregelmatig bloedverlies. Soms kan een behandeling hiervoor noodzakelijk zijn, bijvoorbeeld een spiraaltje, een kleine operatie in dagbehandeling of het laten verwijderen van de baarmoeder . Vanaf percentage van risico op het optreden van vloeistoornissen zou u kiezen verwijdering van de baarmoeder? Dus: hoe hoog moet het risico op vloeistoornissen zijn om te kiezen voor een verwijdering van de baarmoeder in het geval van een baarmoederverzakking?
$\square 5 \%$
(5 op 100 vrouwen)
$\square 10 \%$
(10 op de 100 vrouwen)
$\square \quad 15 \%$
(15 op de 100 vrouwen)
(20 op de 100 vrouwen)
$\square 25 \%$
(25 op de 100 vrouwen)
(30 op de 100 vrouwen)
(35 op de 100 vrouwen)
(40 op de 100 vrouwen)
(45 op de 100 vrouwen)
$\square$
(50 op de 100 vrouwen)
$\square \quad 50 \%$
$\square$ Meer dan $50 \%$
$\square$ Ik kies bij dit probleem niet voor een baarmoederverwijdering
$\square 30 \%$
$\square 35 \%$

\section{VRAGENLIJST LAVA-TRIAL}

1. Wat is uw leeftijd? ... jaar

2. Welke opleiding(en) heeft u voltooid?

$\square$ Basisonderwijs / lagere school (of een deel hiervan)

$\square$ Lager beroepsonderwijs (lts, Ihno, leao, huishoudschool etc.)

$\square$ Mavo, (m)ulo etc

Middelbaar beroepsonderwijs (MTS, MEAO, opleiding tot verpleegkundige etc.)

$\square$ Havo, VWO, gymnasium, mms etc.

$\square$ Hoger beroepsonderwijs (HTS, HEAO, sociale academie)

$\square$ Wetenschappelijk onderwijs (doctoraal examen)

3. a. Wat is uw huidige beroep of zijn uw werkzaamheden? b. Rookt u?

$\square$ Zo ja, hoeveel rookt u per dag? .......... sigaretten/shag per dag

$\square$ Nee

c. Drinkt u alcohol?

$\square$ Zo ja, ......... eenheden per dag

$\square$ Nee

d. Gebruikt u medicijnen? (inclusief de anticonceptiepil)?

$\square \mathrm{Ja}$

$\square$ Nee

Zo ja, kunt u omschrijven welke?

4. a. Heeft u kinderen?
$\square$ Ja
$\square \quad$ Nee (ga door naar vraag 5)

b. Hoeveel kinderen heeft u? ......................... (aantal)

c. Heeft u een keizersnede gehad? Zo ja, hoe vaak?
$\square \mathrm{Ja}$,
keer
$\square$ Nee

d. Heeft $u$ een tangverlossing gehad? Zo ja, hoe vaak?

$\square$ Ja, ................. keer $\square$ Nee

e. Heeft u een vacuümcup verlossing gehad? Zo ja, hoe vaak?

$\square \mathrm{Ja}, \ldots \ldots \ldots \ldots \ldots . . . .$. keer $\quad \square$ Nee

f. Heeft u een 'knip' gehad bij de bevalling? Zo ja, hoe vaak?

$\square$ Ja, ................ keer $\quad \square$ Nee

g. Bent u 'ingescheurd' bij de bevalling? Zo ja, hoe vaak?

$\square$ Ja, ................keer $\quad \square$ Nee 
h. Wanneer was u laatste bevalling? (dag/maand/jaar)

i. Hoe oud was u tijdens de eerste bevalling? (leeftijd in jaren)

Bij de volgende vraag lopen de antwoordcategorieën op van 1 (erg slecht) tot 6 (uitstekend). Wilt $u$ het getal omcirkelen dat het meest op $u$ van toepassing is?

5. Hoe zou u uw algehele kwaliteit van leven gedurende de afgelopen week beoordelen?

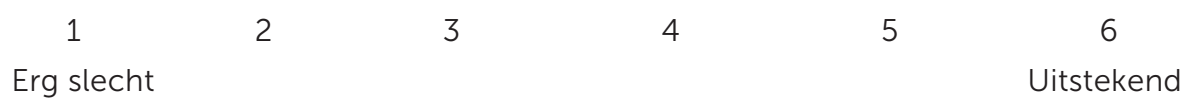

\section{EQ-5D (EURO QOL-5D)}

De volgende 5 vragen hebben betrekking op uw huidige gezondheidstoestand. Kruis bij elke vraag de zin aan die het beste past bij uw eigen gezondheidstoestand vandaag.

6. a. Mobiliteit (slechts één antwoord aankruisen)
$\square \quad$ Ik heb geen problemen met lopen.
$\square \quad$ Ik heb enige problemen met lopen
$\square$ Ik ben bedlegerig.

b. Zelfzorg (slechts één antwoord aankruisen)

$\square \quad \mathrm{Ik}$ heb geen problemen om mijzelf te wassen of aan te kleden.

$\square \quad \mathrm{Ik}$ heb enige problemen om mijzelf te wassen of aan te kleden.

$\square \quad \mathrm{Ik}$ ben niet in staat mijzelf te wassen of aan te kleden.

c. Dagelijkse activiteiten, bijv. werk, studie, huishouden, gezins- en vrijetijdsactiviteiten (slechts één antwoord aankruisen)

$\square \quad$ Ik heb geen problemen met mijn dagelijkse activiteiten

$\square \quad$ Ik heb enige problemen met mijn dagelijkse activiteiten.

$\square$ Ik ben niet in staat mijn dagelijkse activiteiten uit te voeren

d. Pijn / klachten (slechts één antwoord aankruisen)

$\square \quad \mathrm{Ik}$ heb geen pijn of andere klachten.

$\square \quad$ Ik heb matige pijn of andere klachten

$\square \quad$ Ik heb zeer ernstige pijn of andere klachten. e. Stemming (slechts één antwoord aankruisen)

$\square \quad$ Ik ben niet angstig of somber.

$\square \quad$ Ik ben matig angstig of somber

$\square$ Ik ben erg angstig of somber

f. Gezondheidstoestand op meetschaal

Om u te helpen bij het aangeven hoe goed of hoe slecht een gezondheidstoestand

is, hebben we een meetschaal gemaakt, te vergelijken met een thermometer.

Op deze schaal betekent ' 100 ' de beste gezondheidstoestand die u zich kunt voorstellen en ' $\mathrm{O}$ ' de slechtste. Wil u met het zetten van een kruis op de horizontale lijn aangeven hoe u uw eigen gezondheidstoestand op dit moment waardeert?

\section{ALGEHELE GEZONDHEID}

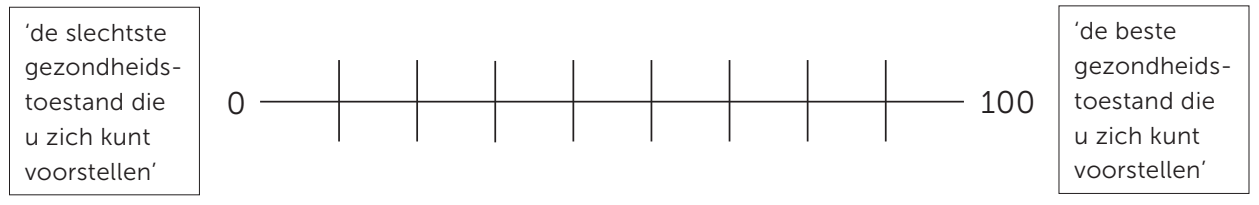

\section{UDI-12 (UROGENITAL DISTRESS INVENTORY)}

Vrouwen met ongewenst urineverlies hebben aangegeven dat ze de volgende klachten hadden. Kunt $\mathrm{u}$ aangeven welke klachten $\mathrm{u}$ op dit moment ook heeft, en hoeveel last $\mathrm{u}$ daarvan heeft? Ook als $\mathrm{u}$ geen klachten denkt te hebben, is het belangrijk dat $\mathrm{u}$ alle vragen beantwoordt. Kruis het juiste antwoord aan.

7. a. Vindt $u$ dat $u$ vaak moet plassen?
$\square \mathrm{Ja}$
$\square$ Nee (ga naar vraag 8)
b. Zo ja, hoeveel last heeft u hier van?
$\square$ Helemaal niet $\square$ Een beetje
Nogal $\square$ Heel erg

8. a. Als u moet plassen, voelt $\mathrm{u}$ dan altijd een sterke aandrang?

$\square$ Ja $\square$ Nee (ga naar vraag 9)

b. Zo ja, hoeveel last heeft u hier van?

$\square$ Helemaal niet $\square$ Een beetje

Nogal

Heel erg 
9. a. Heeft $u$ ongewenst urineverlies als $u$ aandrang voelt om te plassen? $\square$ Ja $\square$ Nee (ga naar vraag 10)

b. Zo ja, hoeveel last heeft u hier van?
$\square$ Helemaal niet
$\square$ Een beetje
$\square$ Nogal
$\square$ Heel erg

c. Zo ja, hoe vaak verliest $\mathrm{u}$ ongewild urine?

$\square$ dagelijks

$\square$ paar keer per week

$\square 1$ keer per week

$\square 1$ keer per maand

$\square 1$ keer per jaar

10. a. Heeft $u$ ongewenst urineverlies bij lichamelijke inspanning, hoesten of niezen? $\square$ Ja $\square$ Nee (ga naar vraag 11)

b. Zo ja, hoeveel last heeft u hier van?
$\square$ Helemaal niet
$\square$ Een beetje
Nogal $\square$ Heel erg

c. Zo ja, hoe vaak verliest $\mathrm{u}$ ongewild urine?

$\square$ dagelijks

$\square$ paar keer per week

$\square 1$ keer per week

$\square 1$ keer per maand

$\square 1$ keer per jaar

11. a. Heeft u moeite uw blaas leeg te plassen?

$\square$ Ja $\square$ Nee (ga naar vraag 12)

b. Zo ja, hoeveel last heeft u hier van?

$\square$ Helemaal niet $\square$ Een beetje

$\square$ Nogal $\quad \square$ Heel erg

12. a. Heeft $\mathrm{u}$ wel eens het gevoel dat uw blaas na het plassen niet helemaal leeg is?

$\square$ Ja $\square$ Nee (ga naar vraag 13)

b. Zo ja, hoeveel last heeft u hier van?

$\square$ Helemaal niet $\square$ Een beetje $\quad \square$ Nogal $\square$ Heel erg

13. a. Heeft u wel eens een drukkend gevoel onder in de buik?
Ja $\square$ Nee (ga naar vraag 14)

b. Zo ja, hoeveel last heeft u hier van?
14. a. Heeft $u$ wel eens pijn onder in de buik of in de schaamstreek?

$\square$ Ja $\square$ Nee (ga naar vraag 15)

b. Zo ja, hoeveel last heeft u hier van?

$\square$ Helemaal niet $\square$ Een beetje $\quad \square$ Nogal $\quad \square$ Heel erg

15. a. Heeft u wel eens het gevoel dat er iets uit de vagina stulpt?

$\square$ Ja $\square$ Nee (ga naar vraag 16)

b. Zo ja, hoeveel last heeft u hier van?

$\square$ Helemaal niet $\square$ Een beetje $\quad \square$ Nogal $\square$ Heel erg

16. a. Heeft u wel eens gezien dat er iets uit de vagina stulpt?

$\square$ Ja $\square$ Nee (ga naar vraag 17)

b. Zo ja, hoeveel last heeft u hier van?
$\square$ Helemaal niet
$\square$ Een beetje
$\square$ Nogal
Heel erg

17. Hoe vaak heeft $\mathrm{u}$ het afgelopen jaar een blaasontsteking gehad?
$\square$ nooit
$\square 1$ keer
$\square$ tussen de 2 en 4 keer
$\square$ meer dan 4 keer

18. a. Moet $\mathrm{u}$ 's nachts meer dan 1 keer plassen?
Ja $a$ Nee (ga naar vraag 19)
b. Zo ja, hoeveel last heeft u hier van?
$\square$ Helemaal niet $\square$ Een beetje
Nogal
Heel erg

\section{DDI (DEFECATORY DISTRESS INVENTORY)}

De volgende verschijnselen zijn beschreven door vrouwen met klachten van de stoelgang. Geeft $u$ aan welke verschijnselen $u$ tegenwoordig herkent en hoeveel last $u$ daarvan heeft. Kruis het juiste antwoord aan.

19. a. Heeft u minder dan driemaal per week ontlasting?
$\square$
$\square \quad$ Nee (ga naar vraag 20)
b. Zo ja, hoeveel last heeft u hier van?
$\square$ Helemaal niet $\square$ Een beetje
$\square$ Nogal
Heel erg 
20. a. Moet $u$ om ontlasting te krijgen in meer dan een kwart van de keren persen? $\square$ Ja $\square \quad$ Nee (ga naar vraag 21)

b. Zo ja, hoeveel last heeft u hier van?
$\square$ Helemaal niet
$\square$ Een beetje
$\square$ Nogal
$\square$ Heel erg

21. a. Heeft $u$ wel eens aandrang tot ontlasting terwijl er dan op het toilet geen ontlasting komt?

$\square$ Ja $\quad \square \quad$ Nee (ga naar vraag 22)

b. Zo ja, hoeveel last heeft u hier van?
$\square$ Helemaal niet
$\square$ Een beetje
$\square$ Nogal
Heel erg

22. a. Heeft $u$ wel eens het gevoel dat er iets uit de anus hangt of er iets voor zit? $\square$ Ja $\square$ Nee (ga naar vraag 23)

b. Zo ja, hoeveel last heeft u hier van?
$\square$ Helemaal niet
$\square$ Een beetje
$\square$ Nogal
Heel erg

23. a. Ervaart u pijn tijdens de aandrang tot ontlasting?
$\square$
$\square$ Nee (ga naar vraag 24)b. Zo ja, ho

$\square$ Helemaal niet

$\square$ Nogal

$\square$ Heel erg

24. a. Ervaart u pijn tijdens of vlak na de ontlasting?
$\square$ Ja $\square$ Nee (ga naar vraag 25)
b. Zo ja, hoeveel last heeft u hier van?
$\square$ Helemaal niet $\square$ Een beetje
$\square$ Nogal
$\square$ Heel erg

25. a. Verliest $u$ wel eens dunne ontlasting zonder dat $u$ daar controle over heeft?
$\square$
c. Hoe vaak komt het voor?
$\square$ dagelijks
$\square$ paar keer per week
$\square 1$ keer per week
$\square 1$ keer per maand
$\square 1$ keer per jaar

$\square$ Nogal

$\square$ Heel erg
26. a. Verliest $u$ wel eens vaste ontlasting zonder dat $u$ daar controle over heeft? $\square$ Ja $\square$ Nee (ga naar vraag 27)

b. Zo ja, hoeveel last heeft u hier van?
$\square$ Helemaal niet $\square$ Een beetje
$\square$ Nogal
$\square$ Heel erg

c. Hoe vaak komt het voor?

$\square$ dagelijks

$\square$ paar keer per week

$\square \quad 1$ keer per week

$\square 1$ keer per maand

$\square 1$ keer per jaar

27. a. Verliest $u$ wel eens windjes zonder dat $u$ daar controle over heeft? $\square$ Ja $\square$ Nee (ga naar vraag 28)

b. Zo ja, hoeveel last heeft u hier van?
$\square$ Helemaal niet $\square$ Een beetje
$\square$ Nogal $\square$ Heel erg

c. Hoe vaak komt het voor?

$\square$ dagelijks

$\square$ paar keer per week

$\square 1$ keer per week

$\square \quad 1$ keer per maand

$\square 1$ keer per jaar

28. a. Moet $u$ wel eens via de schede mee drukken om ontlasting te krijgen? $\square$ Ja $\quad \square \quad$ Nee (ga naar vraag 29)

b. Zo ja, hoeveel last heeft $\mathrm{u}$ hier van?
$\square$ Helemaal niet
$\square$ Een beetje
$\square$ Nogal
$\square$ Heel erg

29. a. Moet $\mathrm{u}$ de ontlasting wel eens met de vingers via de anus verwijderen? $\square$ Ja $\square$ Nee (ga naar vraag 30)

b. Zo ja, hoeveel last heeft u hier van?

$\square$ Helemaal niet $\square$ Een beetje $\quad \square$ Nogal $\square$ Heel erg 


\section{IIQ (INCONTINENCE IMPACT QUESTIONNAIRE)}

Sommige vrouwen vinden dat ongewenst urineverlies hun activiteiten, relaties en gevoelens kunnen beïnvloeden. De vragen in onderstaande lijst gaan over aspecten van uw leven die door uw probleem beïnvloed of veranderd kunnen zijn. Geef voor iedere vraag het antwoord dat het beste beschrijft hoe zeer uw activiteiten, relaties en gevoelens beïnvloed worden door uw urineverlies en/of verzakking en/of problemen met de ontlasting. Kruis het juiste antwoord aan.

Hoeveel invloed heeft ongewenst urineverlies en/of verzakking en/of problemen met de ontlasting gehad op:

30. Uw vermogen om huishoudelijk werk te doen (koken, schoonmaken, wassen)
$\square$ Helemaal niet
$\square$ Een beetje
$\square$ Nogal
$\square$ Heel erg

31. Uw vermogen om klein onderhoud of reparaties te verrichten in en om het huis
$\square$ Helemaal niet
$\square$ Een beetje
$\square$ Nogal
$\square$ Heel erg

32. Boodschappen doen en winkelen
$\square$ Helemaal niet
$\square$ Een beetje
Nogal
Heel erg

33. Reizen met auto en openbaar vervoer over een afstand van minder dan 20 minuten
$\square$ Helemaal niet
$\square$ Een beetje
$\square$ Nogal
$\square$ Heel erg

34. Ergens naar toe gaan als u niet helemaal zeker weet of er daar toiletten zijn
$\square$ Helemaal niet
$\square$ Een beetje
$\square$ Nogal
$\square$ Heel erg

35. Bezoek krijgen van vrienden en kennissen
$\square$ Helemaal nie
$\square$ Een beetj
$\square$ Nogal
Heel erg

36. Relaties met vrienden en kennissen
$\square$ Helemaal niet
$\square$ Een beetje
$\square$ Nogal
$\square$ Heel erg

37. Vermogen om een seksuele relatie te hebben
$\square$ Helemaal niet
$\square$ Een beetje
$\square$ Nogal
Heel erg

38. Geestelijke / emotionele gezondheid
$\square$ Helemaal niet
$\square$ Een beetje
$\square$ Nogal
Heel erg

39. Wordt $\mathrm{u}$ in uw activiteiten beperkt door angst dat anderen $\mathrm{u}$ ruiken?
$\square$ Helemaal niet
$\square$ Een beetje
$\square$ Nogal
$\square$ Heel erg

Heeft u als gevolg van uw probleem de volgende gevoelens?

40. Nervositeit of ongerustheid
$\square$ Helemaal niet
Een beetje
$\square$ Nogal
Heel erg

41. Frustratie
$\square$ Helemaal niet
Een beetje
$\square$ Nogal
$\square$ Heel erg

42. Zich gegeneerd voelen
$\square$ Helemaal niet
Een beetje
Nogal
Heel erg

\section{SEKSUELE DYSFUNCTIES}

De volgende vragen gaan over de seksualiteit. Het is de bedoeling dat $u$ bij het antwoorden denkt aan de situatie van de afgelopen maand. Kruis voor $u$ het meest passende antwoord aan.

43. a. Heeft u wel eens seksueel contact met uw partner? (denk hierbij aan alle vormen van seksueel contact en niet alleen aan geslachtsgemeenschap)

$$
\square \text { Ja } \square \text { Nee }
$$

b. Zo ja, hoe tevreden bent u daarover?
$\square$ Helemaal niet $\square$ Een beetje
$\square$ Nogal $\square$ Heel erg
c. Zo nee, hoe vervelend vindt $u$ dit?
$\square$ Helemaal niet $\square$ Een beetje
$\square$ Nogal $\quad \square$ Heel erg

44. Hoe vaak heeft u geslachtsgemeenschap?
$\square$ Nooit
$\square$ Minder dan 1 keer per maand
$\square 1$ tot 2 keer per maand
$\square 1$ keer per week
$\square$ Meerdere keren per week

45. a. Verliest u wel eens urine tijdens de geslachtsgemeenschap?
$\square$ Nee
$\square$ Niet van toepassing, geen seks
b. Zo ja, hoe veel last hebt u hiervan?
$\square$ Helemaal niet $\square$ Een beetje
Nogal
Heel erg 
46. a. Ervaart u pijn tijdens de geslachtsgemeenschap?
$\square$ Ja $\square$ Nee
$\square \quad$ Niet van toepassing, geen seks
b. Zo ja, hoe veel last hebt u hiervan?
$\square$ Helemaal niet $\square$ Een beetje $\square$ Nogal $\square$ Heel erg

47. a. Is de vagina zo nauw dat geslachtsgemeenschap daardoor niet mogelijk is? $\square$ Ja $\quad \square$ Nee $\quad \square$ Niet van toepassing, geen seks

b. Zo ja, hoeveel last heeft u hier van?
$\square$ Helemaal niet
$\square$ Een beetje
$\square$ Nogal
$\square$ Heel erg

\section{SEKSUEEL FUNCTIONEREN BIJ VERZAKKING EN ONGEWENST URINEVERLIES}

\section{(KORTE VERSIE: PISQ-12)}

Voor $u$ ligt een lijst met vragen over het seksueel leven van $u$ en uw partner. Alle informatie is strikt vertrouwelijk. Bij het beantwoorden van de vragen gaat u uit van uw seksueel leven van de afgelopen 6 maanden.

48. Hoe vaak voelt u seksueel verlangen? Dit gevoel kan bestaan uit het willen hebben van seks, het voelen van frustratie door het gebrek aan seks, enz.
$\square$ Dagelijks
$\square 1$ - 3 keer per week
$\square \quad 1$ - 3 keer per maand
$\square$ Minder dan een keer per maand
$\square$ Nooit

49. Ervaart $u$ altijd een orgasme als u geslachtsgemeenschap heeft met uw partner?
$\square$ Altijd
$\square$ Meestal
$\square$ Soms
$\square$ Zelden
$\square$ Nooit
$\square \quad$ Niet van toepassing, geen seks

50. Voelt u zich seksueel opgewonden als u seksueel actief bent met uw partner?
$\square$ Altijd
$\square$ Meestal
$\square$ Soms
$\square$ Zelden
$\square$ Nooit
$\square \quad$ Niet van toepassing, geen seks

51. Bent u tevreden met de variatie van de seksuele activiteiten in uw huidige seksueel leven?
$\square$ Altijd
$\square$ Meesta
$\square$ Soms
$\square$ Zelden
$\square$ Nooit
$\square$ Niet van toepassing, geen seks

52. Ervaart u pijn tijdens geslachtsgemeenschap?
$\square$ Altijd
$\square$ Meesta
$\square$ Soms
$\square$ Zelden
$\square$ Nooit
$\square \quad$ Niet van toepassing, geen seks

53. Bent $\mathrm{u}$ incontinent voor urine tijdens seksuele activiteit?
$\square$ Altijd
$\square$ Meestal
$\square$ Soms
$\square$ Zelden
$\square$ Nooit
$\square \quad$ Niet van toepassing, geen seks 
54. Wordt u in uw seksuele activiteit beperkt door angst voor incontinentie (ontlasting en/of urine?
$\square$ Altijd
$\square$ Meestal
$\square$ Soms
$\square$ Zelden
$\square$ Nooit
$\square \quad$ Niet van toepassing, geen seks

55. Vermijdt u geslachtsgemeenschap vanwege een uitstulping in de vagina (verzakking)?
$\square$ Altijd
$\square$ Meestal
$\square$ Soms
$\square$ Zelden
$\square$ Nooit
$\square$ Niet van toepassing, geen seks

56. Heeft $u$, als $u$ seks heeft met uw partner, negatieve emotionele gevoelens, zoals angst, afkeer, schaamte of schuldgevoel?
$\square$ Altijd
$\square$ Meestal
$\square$ Soms
$\square$ Zelden
$\square$ Nooit
$\square$ Niet van toepassing, geen seks

57. 57. Heeft uw partner problemen met erecties die uw seksuele activiteiten beïnvloeden?
$\square$ Altijd
$\square$ Meestal
$\square$ Soms
$\square$ Zelden
$\square$ Nooit
$\square \quad$ Niet van toepassing, geen seks

58. Heeft uw partner een probleem met voortijdig klaarkomen die uw seksuele activiteiten beïnvloeden?
$\square$ Altijd
$\square$ Meestal
$\square$ Soms
$\square$ Zelden
$\square$ Nooit
$\square \quad$ Niet van toepassing, geen seks

59. Hoe intens waren de orgasmen in de afgelopen 6 maanden in vergelijking met orgasmen in het verleden?
$\square$ Veel minder intens
$\square$ Minder intens
$\square$ Dezelfde intensiteit
$\square$ Meer intens
$\square$ Veel meer intens
$\square$ Niet van toepassing, geen seks

\section{SHORT FORM HEALTH SURVEY (SF-36)}

De volgende vragenlijst gaat over uw standpunten ten aanzien van uw gezondheid. Met behulp van deze gegevens kan worden bijgehouden hoe $u$ zich voelt en hoe goed $\mathrm{u}$ in staat bent uw gebruikelijke bezigheden uit te voeren. Als $\mathrm{u}$ niet zeker weet hoe $\mathrm{u}$ een vraag moet beantwoorden, geef dan het best mogelijke antwoord.

1. Wat vindt $u$, over het algemeen genomen, van uw gezondheid? (kruis één vakje aan)
$\square$ Uitstekend
$\square$ Zeer goed
$\square$ Goed
$\square$ Matig
$\square$ Slecht 
2. In vergelijking met een jaar geleden, joe zou u nu uw gezondheid in het algemeen beoordelen? (kruis één vakje aan)

$\square$ Veel beter nu dan een jaar geleden

$\square$ Wat beter nu dan een jaar geleden

$\square$ Ongeveer hetzelfde nu als een jaar geleden

$\square$ Wat slechter nu dan een jaar geleden

$\square$ Veel slechter nu dan een jaar geleden

3. De volgende vragen gaan over bezigheden die u misschien doet op een doorsnee dag. Wordt u door uw gezondheid op dit moment beperkt bij deze bezigheden? Zo ja, in welke mate? (kruis één vakje aan op elke regel?

\begin{tabular}{lccc}
\hline & $\begin{array}{c}\text { Ja, ernstig } \\
\text { beperkt }\end{array}$ & $\begin{array}{c}\text { Ja, een beetje } \\
\text { beperkt }\end{array}$ & $\begin{array}{c}\text { Nee, helemaal } \\
\text { niet beperkt }\end{array}$ \\
\hline a. Forse inspanning & $\square$ & $\square$ & $\square$ \\
$\begin{array}{l}\text { Zoals hardlopen, zware voorwerpen tillen } \\
\text { b. Matige inspanning }\end{array}$ & & & \\
Zoals het verplaatsen van een tafel,stofzuigen, fietsen & & & \\
c. Tillen of boodschappen dragen & $\square$ & $\square$ & $\square$ \\
d. Een paar trappen oplopen & $\square$ & $\square$ & $\square$ \\
e. Eén trap oplopen & $\square$ & $\square$ & $\square$ \\
f. Buigen, knielen of bukken & $\square$ & $\square$ & $\square$ \\
g. Meer dan een kilometer lopen & $\square$ & $\square$ & $\square$ \\
h. Een halve kilometer lopen & $\square$ & $\square$ & $\square$ \\
i. Honderd meter lopen & $\square$ & $\square$ & $\square$ \\
j. Uzelf wassen of aankleden & $\square$ & $\square$ & $\square$ \\
\hline
\end{tabular}

4. Heeft in de afgelopen 4 weken, een van de volgende problemen bij uw werk of andere dagelijkse bezigheden gehad, ten gevolge van uw lichamelijke gezondheid? (kruis één vakje aan op elke regel)

\begin{tabular}{lcc}
\hline & $\mathrm{Ja}$ & Nee \\
\hline a. U heeft minder tijd kunnen besteden aan werk of ander bezigheden & $\square$ & $\square$ \\
b. U heeft minder bereikt dan u zou willen & $\square$ & $\square$ \\
c. U was beperkt in het soort werk of het soort bezigheden & $\square$ & $\square$ \\
$\begin{array}{l}\text { d. U had moeite met het extra werk of andere bezigheden (het kostte u bijvoorbeeld extra } \\
\text { inspanning) }\end{array}$ & $\square$ & $\square$ \\
\hline
\end{tabular}

5. Heeft $\mathrm{u}$ in de afgelopen 4 weken, een van de volgende problemen ondervonden bij uw werk of andere dagelijkse bezigheden ten gevolge van emotionele problemen (zoals depressieve of angstige gevoelens)? (kruis één vakje aan op elke regel)
6. In hoeverre hebben uw lichamelijke gezondheid of emotionele problemen u gedurende de afgelopen 4 weken gehinderd in uw normale omgang met familie, vrienden of buren, of bij activiteiten in groepsverband? (kruis één vakje aan)
$\square$ Helemaal niet
$\square$ Enigszins
$\square$ Nogal
$\square$ Veel
$\square$ Heel erg veel

7. Hoeveel lichamelijke pijn heeft $u$ de afgelopen 4 weken gehad (kruis één vakje aan)
$\square$ Geen
$\square$ Heel licht
$\square$ Licht
$\square$ Nogal
$\square$ Ernstig
$\square$ Heel ernstig

8. In welke mate bent $u$ de afgelopen 4 weken door pijn gehinderd in uw normale werk (zowel werk buitenshuis als huishoudelijk werk)? (kruis één vakje aan)
$\square$ Helemaal niet
$\square$ Enigszins
$\square$ Nogal
$\square$ Veel
$\square$ Heel erg veel

9. Deze vragen gaan over hoe zich voelt en hoe het met $u$ ging in de afgelopen 4 weken. Wilt u a.u.b. bij elke vraag het antwoord geven dat het best benadert hoe u zich voelde. (kruis één vakje aan op elke regel)

\begin{tabular}{lcccccc}
\hline & Voortdurend & Meestal & Vaak & Soms & Zelden & Nooit \\
\hline a. voelde u zich levenslustig? & $\square$ & $\square$ & $\square$ & $\square$ & $\square$ & $\square$ \\
b. voelde u zich erg zenuwachtig? & $\square$ & $\square$ & $\square$ & $\square$ & $\square$ & $\square$ \\
c. zat u zo erg in de put dat niet u kon opvrolijken? & $\square$ & $\square$ & $\square$ & $\square$ & $\square$ & $\square$ \\
d. voelde u zich kalm en rustig & $\square$ & $\square$ & $\square$ & $\square$ & $\square$ & $\square$ \\
e. voelde u zich erg energiek? & $\square$ & $\square$ & $\square$ & $\square$ & $\square$ & $\square$ \\
f. voelde u zich neerslachtig en somber? & $\square$ & $\square$ & $\square$ & $\square$ & $\square$ & $\square$ \\
g. voelde u zich uitgeblust? & $\square$ & $\square$ & $\square$ & $\square$ & $\square$ & $\square$ \\
h. voelde u zich gelukkig? & $\square$ & $\square$ & $\square$ & $\square$ & $\square$ & $\square$ \\
i. voelde u zich moe? & $\square$ & $\square$ & $\square$ & $\square$ & $\square$ & $\square$ \\
\hline
\end{tabular}


10. Hoe vaak hebben uw lichamelijke gezondheid of emotionele problemen u gedurende de afgelopen 4 weken gehinderd bij uw sociale activiteiten (zoals vrienden of familie bezoeken etc.)? (kruis één vakje aan)
$\square$ Voortdurend
$\square$ Meestal
$\square$ Soms
$\square$ Zelden
$\square$ Nooit

11. Hoe JUIST of ONJUIST is elk van de volgende uitspraken voor u? (kruis één vakje aan op iedere elke regel)

\begin{tabular}{lccccc}
\hline & $\begin{array}{c}\text { Volkomen } \\
\text { juist }\end{array}$ & $\begin{array}{c}\text { Groten- } \\
\text { deels juist }\end{array}$ & $\begin{array}{c}\text { Weet } \\
\text { ik niet }\end{array}$ & $\begin{array}{c}\text { Groten- } \\
\text { deels onjuist }\end{array}$ & $\begin{array}{c}\text { Volkomen } \\
\text { onjuist }\end{array}$ \\
\hline a. Ik lijk gemakkelijker ziek te worden dan & $\square$ & $\square$ & $\square$ & $\square$ & $\square$ \\
andere mensen & & & & & \\
$\begin{array}{l}\text { b. Ik ben net zo gezond als andere mensen } \\
\text { die ik ken }\end{array}$ & $\square$ & $\square$ & $\square$ & $\square$ & $\square$ \\
$\begin{array}{l}\text { c. Ik verwacht dat mijn gezondheid achteruit } \\
\text { zal gaan }\end{array}$ & $\square$ & $\square$ & $\square$ & $\square$ & $\square$ \\
d. Mijn gezondheid is uitstekend & $\square$ & $\square$ & $\square$ & $\square$ & $\square$ \\
\hline
\end{tabular}

\section{PUBLICATIONS}

2020 IJsselmuiden MN van, Oudheusden AMJ van, Veen J, Pol G van de, Vollebregt A, Radder CM, Housmans S, Kuijk SMJ van, Deprest J, Bongers MY, Eijndhoven HWF van. Hysteropexy in the treatment of uterine prolapse Stage 2 or higher: laparoscopic sacrohysteropexy versus sacrospinous hysteropexy-a multicentre randomised controlled trial (LAVA trial). BJOG 2020; doi: 10.1111/1471-0528.16242.

2020 IJsselmuiden MN van, Lecomte-Grosbras PM, Witz JF, Brieu M, Cosson M, Eijndhoven HWF van. Dynamic magnetic resonance imaging to quantify pelvic organ mobility after treatment for uterine descent: differences between surgical procedures. Int Urogynecol J. 2020 doi: 10.1007/s00192-02004278-5.

2018 IJsselmuiden MN van, Detollenaere RJ, Gerritse MBE, Kluivers KB, Bongers MY, Eijndhoven HWF van. Dutch women's attitudes towards hysterectomy and uterus preservation in surgical treatment of pelvic organ prolapse. Eur J Obstet Gynecol Reprod Biol 2018;220:79-83

2017 Coolen AMW, IJsselmuiden MN van, Oudheusden AMJ van, Veen J, Eijndhoven HWF van, Mol BWJ, Roovers JP, Bongers MY. Laparoscopic sacrocolpopexy versus vaginal sacrospinous fixation for vaginal vault prolapse, a randomized controlled trial: SALTO-2 trial, study protocol. BMC Womens Health 2017;17(1):52

2016 IJsselmuiden MN van, Erkelens JA, Eijndhoven HWF van. Praktijkvariatie in behandeling van prolaps en urine-incontinentie: een maat voor de kwaliteit van zorg in Nederland? NTOG mei 2016;129:180-85

2015 IJsselmuiden MN van, Detollenaere RJ, Kampen MY, Engberts MK, Eijndhoven HWF van. Practice pattern variation in surgical management of pelvic organ prolapse and urinary incontinence in The Netherlands. Int Urogynecol J 2015;26(11):1649-56

2015 IJsselmuiden MN van, Kerkhof MH, Schellart RP, Bongers MY, Spaans WA, Eijndhoven HWF van. Variation in practice of laparoscopic sacrohysteropexy and laparoscopic sacrocolpopexy for treatment of pelvic organ prolapse: a Dutch survey. Int Urogynecol J 2015 May 26;26(5):757-64

2014 IJsselmuiden MN van, Coolen ALWM, Detollenaere RJ, Boon J den, Bongers MY, Pol G van de, Vollebregt A, Eijndhoven HWF van. Hysteropexy in treatment of uterine descent stage 2 or higher: a multicenter randomized controlled trial comparing laparoscopic sacrohysteropexy with vaginal sacrospinous hysteropexy (LAVA-trial). BMC Womens Health, 2014 Sep 17;14:112

2013 IJsselmuiden MN van, Bekhof J, Peek AM. Een neonaat met het "blueberry 
muffin syndroom". Ned Tijdschr Geneesk. 2013:157(33):A6460

2011 Aukes AM, IJsselmuiden MN van, Wiegman MJ, Postma IR, Veen TR van, Roos N, Graaf AM van der, Zeeman GG. Het maternale brein: oorzaken van eclampsie en de langetermijngevolgen. NTOG. 2011 April; 124:116-120.

2011 IJsselmuiden MN van, Wiegman MJ, Zeeman GG, Faas MM. The S100B protein in brain tissue and plasma in a preeclampsia rat model. Hypertens Preg. 2011 Apr; 1(2):143-149.

2009 IJsselmuiden MN van, Bos AM, Hoek A, Van Beek AP, Kerstens MN. Lichen Sclerosus en het syndroom van Turner. Ned Tijdschr Geneeskd. 2010;154:A773

\section{DANKWOORD}

Hoera, het is af! Omdat het onmogelijk is om te promoveren zonder de hulp en begeleiding van collega's en steun van vrienden en familie, wil ik in het bijzonder de volgende mensen bedanken:

Allereerst wil ik graag alle vrouwen bedanken die aan de verschillende studies hebben meegedaan. Zonder hun deelname was dit onderzoek niet mogelijk geweest.

Veel dank ben ik verschuldigd aan mijn co-promotor, dr. H.W.F. van Eijndhoven, en promotoren prof. dr. M.Y. Bongers en prof. dr. J. Deprest.

Mijn co-promotor dr. H.W.F van Eijndhoven, beste Hugo, ik kan me ons allereerste gesprek in de koffiekamer in het oude Sophia ziekenhuis nog goed herinneren. Dankzij jou ben ik met dit onderzoek gestart. Vanaf het begin ben je erg betrokken geweest bij de projecten; de artikelen en stukken werden door jou altijd binnen een zeer korte tijd voorzien van commentaar. Ik ben je erg dankbaar voor het vertrouwen dat je in me hebt gehad en de mogelijkheid die je me hebt gegeven om me wetenschappelijk te verdiepen. Ik heb veel bewondering voor de kennis en operatieve vaardigheden die je hebt, en ik kijk er dan ook naar uit om volgend jaar van jou de urogynaecologische praktijk te leren.

Mijn promotor prof. dr. M.Y. Bongers, beste Marlies, in vele opzichten ben je een rolmodel voor mij geweest. Je enthousiasme voor onderzoek werkt enorm motiverend, en een gesprek met jou zorgde er altijd voor dat ik weer met veel energie aan de slag kon gaan. In de laatste fase van het onderzoek hebben we veel contact gehad om de laatste puntjes op de "i" te zetten. Naast interesse in de voortgang van het onderzoek toonde je ook veel interesse in mij als persoon. Dank je wel voor je fijne begeleiding!

Mijn promotor prof. dr. J. Deprest, beste Jan, jouw kritische wetenschappelijk blik en commentaar op de manuscripten zijn zeer waardevol geweest en hebben dit proefschrift naar een hoger niveau gebracht. Bedankt hiervoor!

Leden van de leescommissie, prof. dr. Kruitwagen, dr. Blanker, prof. dr. Bouvy en dr. Hakvoort, dank voor de bereidheid dit proefschrift te beoordelen op wetenschappelijke waarde.

Alle gynaecologen in de deelnemende centra en met name Geerte van de Pol, Celine 
Radder, Astrid Vollebregt, Joggem Veen en Susanne Housmans wil ik bedanken voor hun inzet om patiënten te includeren in de LAVA trial en terug te zien voor follow-up. Ook veel dank voor de betrokken research verpleegkundigen van de deelnemende centra: Leen Mortier, Els Heijman, Rita Kantoor, Anja Ommering, Ingrid 't Hooff en Astrid Ritman. De korte lijntjes met jullie zijn erg fijn, bedankt voor de gastvrijheid en lekkere kopjes koffie tijdens de monitor bezoeken.

Alle mede-auteurs en onderzoekers die meegewerkt hebben aan één of meer projecten wil ik bedanken voor hun inbreng en inspanning. Jan den Boon, samen met Hugo ben jij het brein achter de LAVA-trial. Dank je wel voor je input en begeleiding bij het opzetten van de studie. Sander van Kuijk, wat ben ik blij met jouw hulp bij de statistiek. Samen naar de LAVA-database kijken was de lange treinreis naar Maastricht zeker waard.

Een grote studie uitvoeren in combinatie met de opleiding tot gynaecoloog is alleen mogelijk met een goede research verpleegkundige. Nitolanda van Rijn, ik ben jou veel dank verschuldigd. Dank je wel voor het helpen opzetten van de LAVA en voor je hulp bij de logistiek van de studie. Je bent een harde werker die alles tot in de puntjes regelt, eigenschappen die je goed van pas gaan komen bij je opleiding tot verpleegkundig specialist.

Jody Walraven, ondanks dat we op dezelfde afdeling werken zien we elkaar niet zo vaak. Naast je drukke baan heb je ook nog tijd gevonden om mij te ondersteunen bij de LAVA trial, waarvoor heel veel dank! En dan heb je in de tussentijd ook nog ons gezamenlijk doel volbracht, nu ik nog..

Jans en Marianne, bedankt voor jullie hulp bij de logistiek van de studie.

Ik heb tijdens mijn onderzoek ook veel steun mogen ervaren van de Isala Academie en in het bijzonder wil ik de "Innovatie \& Wetenschapsraad" van de Isala bedanken voor de financiële ondersteuning van de studie. Joep Dille, dank je wel voor je goede hulp bij het opstellen van een begroting voor de LAVA, maar ook bij het regelen van allerlei logistieke uitdagingen waar ik tegenaan liep. Ik ben heel erg blij dat ik enkele maanden fulltime promotie-onderzoek heb mogen doen. Dit was niet mogelijk geweest zonder jouw hulp en zonder de vouchers van het I\&W. Ik ben het I\&W dan ook ongelooflijk erkentelijk; heel veel dank. David de Jong en Saskia Abbes, dankzij jullie hebben wij een goede randomisatiemodule en database kunnen ontwikkelen voor de LAVA trial, hier heb ik veel gemak van gehad. Lonneke Buitenhuis, het monitoren van de deelnemende centra was een leuke bezigheid om samen te doen. Dank je wel voor je punctualiteit en je kritische blik!
Het is heel fijn om onderzoeksmaatjes te hebben om af en toe eens te sparren en ideeën uit te wisselen. Renée Detollenaere, wat was het fijn om af en toe wat onderzoeksissues te bespreken en wat heb ik veel aan al je tips gehad. Je bevlogenheid en voortvarendheid heb ik altijd erg bewonderd, en omdat je altijd net een paar stappen voor liep op mij (zowel wetenschappelijk als in de opleiding) ben je in veel opzichten een voorbeeld voor me geweest.

Anne-Lotte Coolen, het is tijd om samen te proosten op onze proefschriften. Enkele jaren na de start van onze studies, en inmiddels beiden twee kinderen verder, hebben we toch maar mooi allebei de eindstreep gehaald.

Anique van Oudheusden, de combinatie opleiding en promoveren is niet altijd gemakkelijk, maar jij slaat je er dapper doorheen! Nog even en dan jij aan de beurt om de SALTO-2 te verdedigen.

Sacha Schulten en Roosje Enklaar, de SAM-studie is al in volle gang. Nog even en dan staan jullie op internationale congressen jullie resultaten te presenteren. Roosje, samen gaan we ons verder verdiepen in de praktijkvariatie van prolapschirurgie in Nederland, dat gaat een leuk onderzoek worden!

Tom en Mirell van de bibliotheek Isala (DISC), bedankt voor de vele fulltexten die jullie voor me opgevraagd hebben met als dieptepunt de fulltext van mijn eigen artikel.

Lieve collega A(N)IOS uit Deventer, Groningen en Zwolle, bedankt voor de prettige samenwerking en de gezelligheid op de werkvloer. Ik ga iedere dag met plezier naar mijn werk, en dat komt ook zeker door de fijne collega's die ik heb.

Alle oud-collega's in het Deventer Ziekenhuis en opleider Paul van der Linden. Mijn eerste jaren als AIOS mocht ik bij jullie werken. Ik heb de samenwerking en begeleiding als erg prettig ervaren en voelde me als een vis in het water in jullie ziekenhuis. Ik zal de telefoontjes van Paul en de bloemen na de geboorte van de kinderen niet snel vergeten, zelfs bijna 2 jaar na vertrek uit het DZ. Ik heb deze betrokkenheid ongelooflijk gewaardeerd.

Mijn oud-collega's in het UMCG en in het bijzonder de opleiders Maaike Oonk en Astrid Cantineau, wil ik graag bedanken voor de fijne tijd die ik als AIOS in de academie heb gehad. Maaike en Astrid, tijdens mijn periode in het UMCG werden jullie opleider, een rol die jullie op het lijf is geschreven. Ik denk met veel plezier terug aan de chipsoverleggen of de korte gesprekken op de gang over opleiding, onderzoek of de kinderen. 
Collega's in de Isala Zwolle, wat is het fijn om weer terug op het nest te zijn. Van semiarts tot ANIOS, onderzoeker en nu de laatste jaren van mijn opleiding: alle stappen heb ik bij jullie gezet. Ik voel me enorm thuis in jullie kliniek, en na een aantal jaren weg te zijn geweest voelt mijn terugkomst als een warm bad. Bedankt voor jullie interesse in mij en in mijn onderzoek. In het bijzonder wil ik de opleiders Ben Cohlen en Walter Kuchenbecker noemen, bedankt voor de fijne opleidingsgesprekken en de mogelijkheden die ik in de Isala van jullie krijg om mezelf verder te ontwikkelen.

Reinie en Marijke, jullie zijn toch echt wel de spil van de afdeling en met een kamer tegenover het koffiezetapparaat zwaai ik iedere dag even naar jullie (wat meestal uitloopt op lang kletsen..). Fijn dat jullie er zijn voor alle logistieke problemen, een luisterend oor, en vooral voor een gezellig praatje.

Lieve familie en vrienden. Hoewel ik niet iedereen persoonlijk kan noemen, wil ik jullie allemaal bedanken voor de interesse in mijn onderzoek maar ook voor alle leuke momenten daarnaast!

Mijn lieve vriendinnen: Annemarie, Elianne, Loes, Margreet en Marieke. Al bijna 20 jaar vormen we een hecht vriendinnengroepje en onze band is, ondanks dat een aantal tijdelijk aan de andere kant van de wereld woonden, alleen maar sterker geworden met de jaren. We hebben allemaal een druk leven, maar we weten elkaar iedere keer weer te vinden. Ik geniet van onze etentjes, weekendjes weg en als hoogtepunt van het jaar het vriendendiner (die nodig weer gepland moet worden!). Jullie vriendschap is mij zeer dierbaar!

Leden van de WES: Sanne en Marieke, wat begon als collega's op de verloskamers is uitgegroeid tot een vriendschap. Altijd gezellig om samen koffie te drinken en bij te kletsen, of om "on tour" te gaan. Laten we snel weer een etentje plannen!

Thijs \& Sanne, Frank-Nico \& Lisette, Laurens \& Annemiek, Wouter \& Jitske-Annie, Jeffrey $\&$ Lyanne, door alle drukke agenda's valt het niet altijd mee om een datum te prikken waarop we elkaar kunnen zien. Maar wat is het altijd gezellig! Laurens en Annemiek, de chocolade kwam op het juiste moment! Lieve Sanne, je bent mijn meest creatieve vriendin, bedankt voor het ontwerpen van de prachtige omslag van dit proefschrift.

Lieve Annemarie, mijn allerbeste vriendinnetje. Onze vriendschap gaat terug tot de basisschool. We hebben dezelfde kijk op het leven en humor die alleen wij begrijpen. Het is een fantastisch gevoel om te weten een vriendin zoals jij te hebben. Daarom ben ik heel trots dat je als paranimf naast me staat.

Lieve Smitjes, ik voelde me vanaf het begin erg welkom in jullie familie. Ik geniet enorm van de gezelligheid en drukte als we met z'n allen zijn. De familie zal weer flink uitgebreid worden dit jaar, ik kijk er naar uit. Hoe meer zielen, hoe meer vreugd! Killy, ik wil jou in het bijzonder bedanken. Door af en toe op de kinderen te passen heb ik wat extra kunnen werken aan dit proefschrift en kon ik de vaart erin houden. Dank je wel hiervoor!

Jakob en Anneke, als "bonus-familie" ben ik blij jullie in mijn leven te hebben. Na het overlijden van papa zijn jullie een grote steun voor mij geweest en jullie zijn een fantastische opa en oma voor onze kinderen. Ik geniet enorm van de weekendjes weg en de gezellige dagjes uit die we gehad hebben samen. Laten we de agenda's (vooral die van ons geloof ik...) weer naast elkaar leggen om weer eens gezellig samen uit eten te gaan.

Jessica, mijn lieve zusje. Ondanks dat we in veel dingen verschillend zijn, hebben we een ijzersterke band. Jouw nuchtere kijk kan soms erg relativerend werken. Ik heb bewondering voor hoe jij je door de afgelopen periode heen hebt geslagen, wat ben je toch krachtig en positief. Ik ben trots op je! Dank je wel dat je altijd voor me klaar staat, en dat je er nu ook voor me bent als paranimf.

Lieve mama, wat ben ik trots op jou. Jouw positieve instelling heeft ervoor gezorgd dat je de draad weer goed op hebt kunnen pakken na het overlijden van papa. Jullie hebben altijd tegen me gezegd dat ik mijn dromen moest nastreven en me gestimuleerd om het beste uit mezelf naar boven te halen. Mede dankzij jullie ben ik alsnog Geneeskunde gaan studeren. Bedankt voor alles!

Mijn mooie lieve kinderen Koen en Fiene, jullie zijn de leukste mensen die ik ken. Ik houd onvoorwaardelijk van jullie, en alleen al naar jullie kijken tovert een glimlach op mijn gezicht. Jullie laten me zien waar het echt om gaat in het leven en wat ultiem geluk is.

Koen, de liefde voor muziek en dansen heb je niet van een vreemde. Ik vind het fantastisch om met jou door de kamer te dansen. Ik hoop dat we dat nog heel lang blijven doen samen. Fiene, wat ben je toch een prachtig meisje en wat heb je een engelengeduld. I $\mathrm{k}$ vind het heerlijk om met je te puzzelen en om boekjes met je te lezen. Jullie zullen een fantastische grote broer en zus zijn. Ik ben apetrots op jullie! 
Robbert, mijn allerliefste en mijn grote liefde. In jou heb ik mijn gelijke gevonden. Wat ben ik blij dat ik jou aan mijn zijde heb. Bedankt voor je steun bij het tot stand komen van dit proefschrift, maar vooral voor je steun bij het najagen van mijn droom om gynaecoloog te worden. Ik kan niet in woorden uitbrengen hoe fantastisch ik je vind, de laatste woorden in dit proefschrift worden dan ook terecht aan jou opgedragen. Ik geniet iedere dag weer van jouw aanwezigheid en van ons mooie gezin. Ik hou van je!

\section{CURRICULUM VITAE}

Mèlanie Nathalie van IJsselmuiden werd op 30 juni 1984 geboren in Havelte. In 2002 behaalde zij haar Atheneum diploma aan de Christelijke Scholengemeenschap Dingstede te Meppel. Na het voltooien van de opleiding "Medische Beeldvormende en Radiotherapeutische Technieken" (HBO-MBRT) heeft zij een half jaar gewerkt als radiodiagnostisch laborant in de Isala Zwolle. In 2007 werd zij toegelaten tot de zij-instroom Geneeskunde aan de Rijksuniversiteit Groningen. Na het behalen van haar artsenbul ging zij in 2012 werken als arts-assistent niet in opleiding (ANIOS) in de Isala Zwolle. Onder leiding van dr. H.W.F. van Eijndhoven werd in de zomer van 2012 een start gemaakt met de LAVA-trial en dit leidde tot een promotie-onderzoek in samenwerking met het Maastricht Universitair Medisch Centrum (begeleiders prof. dr. M.Y. Bongers, prof. dr. J. Deprest en dr. H.W.F. van Eijndhoven). In januari 2015 startte zij met de opleiding tot gynaecoloog in het Deventer Ziekenhuis (opleider dr. P.J.Q. van der Linden). Van oktober 2016 tot september 2018 was zij werkzaam in het Universitair Medisch Centrum Groningen (opleider prof. S.S. Scherjon, dr. M.M. Oonk/ dr. A.E. Cantineau). Sinds september 2019 werkt zij in de Isala Zwolle (opleider dr. B.J. Cohlen), waar zij de laatste jaren van haar opleiding zal besteden aan differentiaties in de voortplantingsgeneeskunde, minimaal-invasieve gynaecologie en urogynaecologie. Mèlanie woont samen met Robbert Smit in Zwolle. Ze zijn de trotse ouders van hun zoon Koen (2016) en dochter Fiene (2018). 

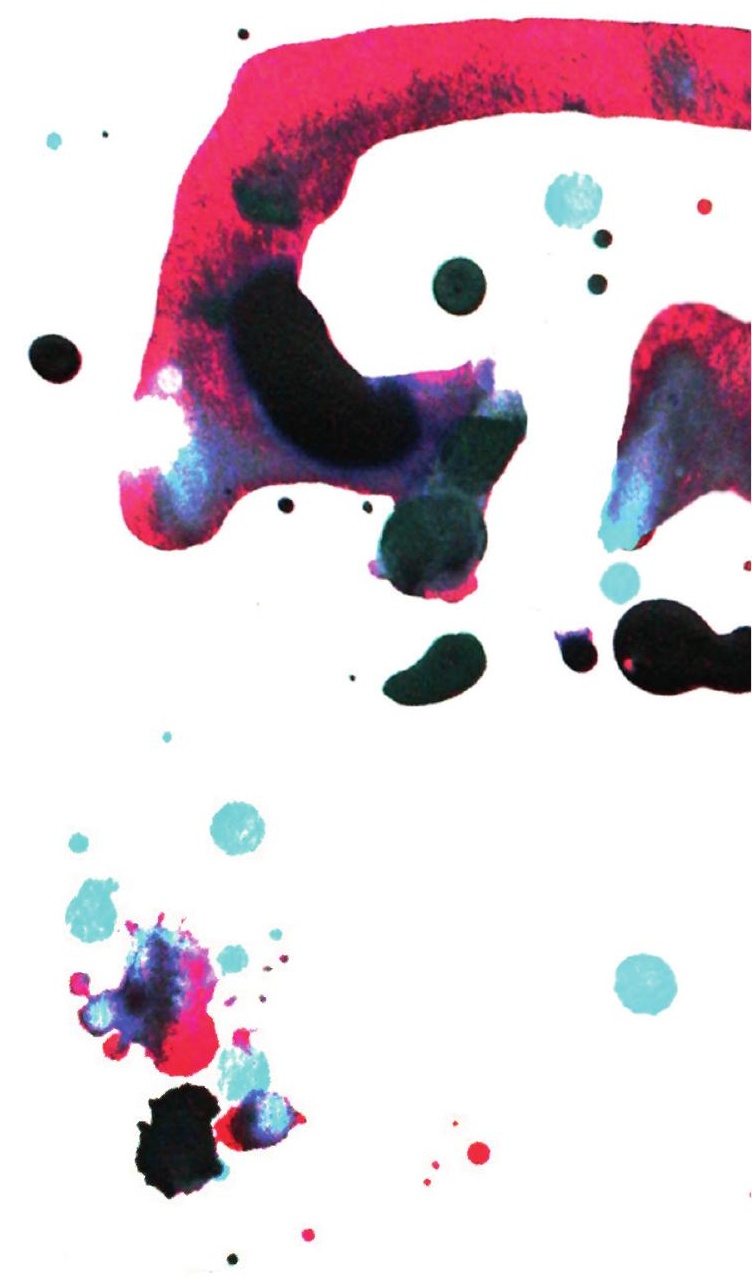

$\nabla$ 